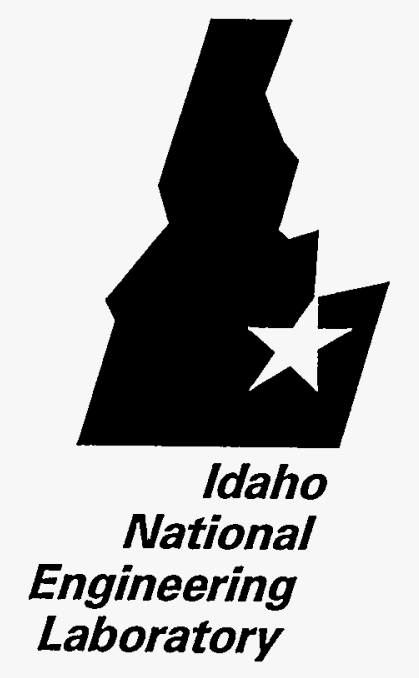

July 1996

\title{
Application of Ultrasound in Solvent Extraction of Nickel and Gallium
}

\author{
PEOEIVEO \\ AUG 211996 \\ O S T I
}

Batric Pesic

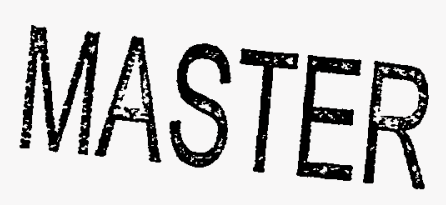

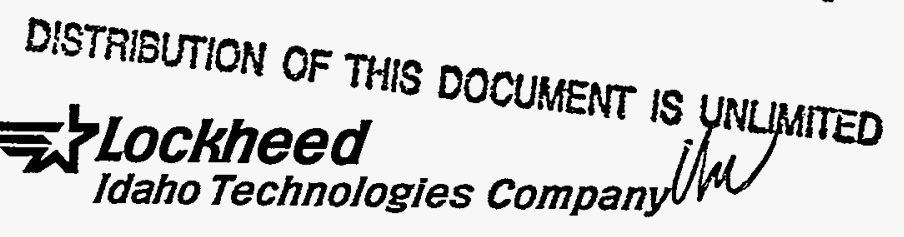


INEL-96/0229

\title{
Application of Ultrasound in Solvent Extraction of Nickel and Gallium
}

\author{
Batric Pesic ${ }^{a}$
}

Published July 1996

\section{Idaho National Engineering Laboratory Lockheed Martin Idaho Technologies Company Idaho Falls, Idaho 83415}

\author{
Prepared for the \\ Interior Department's Bureau of Mines \\ and for the \\ U.S. Department of Energy \\ Assistant Secretary for Environmental Management \\ Under DOE Idaho Operations Office \\ Contract DE-AC07-94ID13223
}

a. University of Idaho, College of Mines and Earth Resources. 


\section{DISCLAIMER}

Portions of this document may be illegible in electronic image products. Images are produced from the best available original document. 


\begin{abstract}
The effects of ultrasound on the rate of solvent extraction of nickel with $\mathrm{Lix} 65 \mathrm{~N}$ and Lix 70, and gallium with Kelex 100 were investigated. These solvent extraction systems are noted by their sluggish nature. Low frequency $(20 \mathrm{kHz})$ ultrasound increased the rates of extraction of nickel by factors of four to seven. The ultrasound had no effect on the final chemical equilibrium. Gallium extraction rates were enhanced with the use of ultrasound by as much as a factor of 15. Again, the ultrasound had no effect on extraction equilibrium. For both nickel and gallium, the enhanced rates were attributed to increased interfacial surface area associated with ultrasonically induced cavitation and microdroplet formation. The stability of the microdroplets permitted intermittent application of ultrasound with corresponding decreases in ultrasonic energy requirements. The lowest energy consumption was observed with short ( 0.25 to $5 \mathrm{~s})$ bursts of high power (41 to $61 \mathrm{~W}$ ) ultrasonic inputs.

The study also provided insight into the factors that affect the complex extraction of gallium from sodium aluminate solutions. The rate controlling step was found to be the dehydration of the gallate ion, $\mathrm{Ga}(\mathrm{OH})_{4}^{-}$, and the first complex formation between gallium and Kelex 100 . Sodium was found to enhance the extraction rate up to a point, beyond which increased concentration was detrimental. Increasing aluminum concentration was found to slow extraction rates. Modifiers and diluents were shown to markedly affect extraction rates even without ultrasound. Ketone modifiers, particularly 2-undecanone, when used with Kermac 470B or Escaid 200 diluents enhanced extraction rates of gallium to the point that the use of ultrasound provided no additional benefits. The positive effects of ketone modifiers for the solvent extraction of gallium had not been previously reported.
\end{abstract}




\section{CONTENTS}

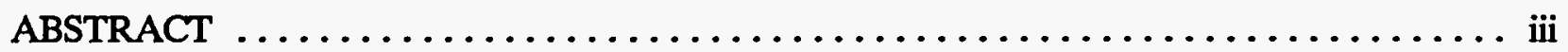

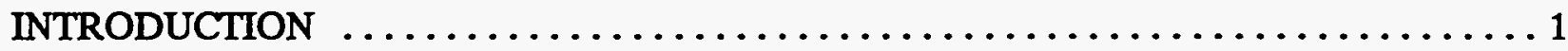

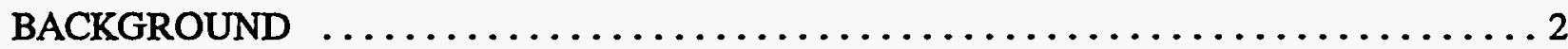

Ore Beneficiation $\ldots \ldots \ldots \ldots \ldots \ldots \ldots \ldots \ldots \ldots \ldots \ldots \ldots \ldots \ldots \ldots \ldots$

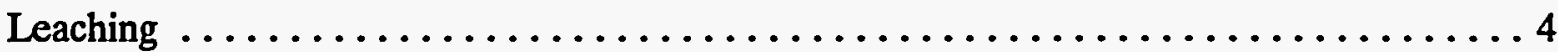

Electrometallurgy $\ldots \ldots \ldots \ldots \ldots \ldots \ldots \ldots \ldots \ldots \ldots \ldots \ldots \ldots \ldots \ldots$

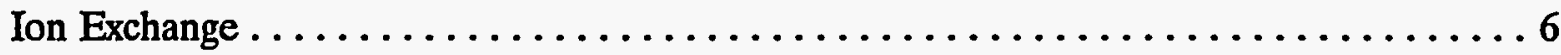

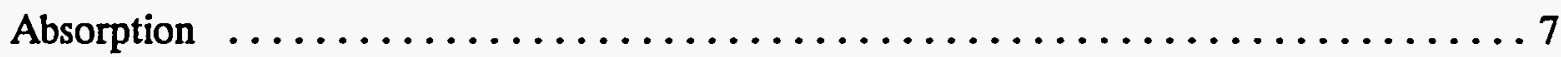

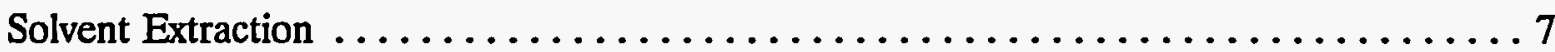

PART I: THE EFFECT OF ULTRASOUND ON THE SOLVENT EXTRACTION OF

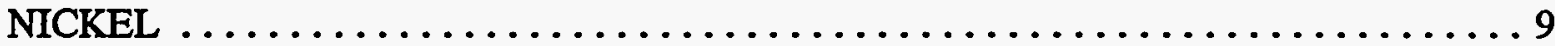

Introduction $\ldots \ldots \ldots \ldots \ldots \ldots \ldots \ldots \ldots \ldots \ldots \ldots \ldots \ldots \ldots \ldots, \ldots$

Experimental Procedure $\ldots \ldots \ldots \ldots \ldots \ldots \ldots \ldots \ldots \ldots \ldots \ldots \ldots \ldots \ldots \ldots$

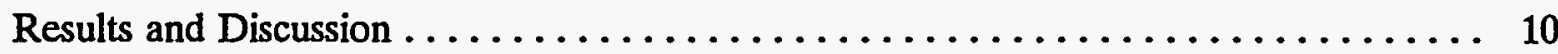

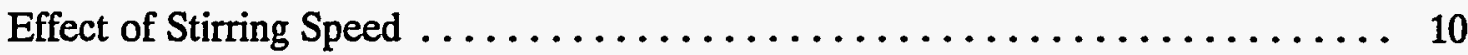

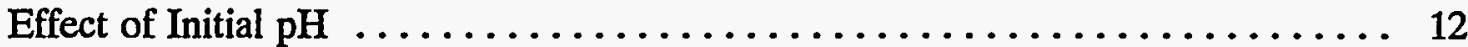

Effect of Temperature $\ldots \ldots \ldots \ldots \ldots \ldots \ldots \ldots \ldots \ldots \ldots \ldots \ldots \ldots \ldots \ldots \ldots \ldots \ldots \ldots, 12$

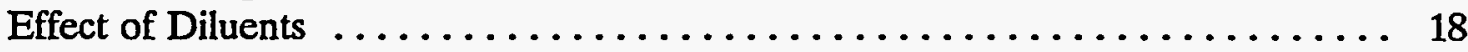

Effect of Initial Nickel Concentration $\ldots \ldots \ldots \ldots \ldots \ldots \ldots \ldots \ldots \ldots, 18$

Effect of Ultrasound on Equilibrium Extraction Loading .............. 20

Extraction of Cobalt and Separation of Cobalt and Nickel $\ldots \ldots \ldots \ldots \ldots \ldots 20$

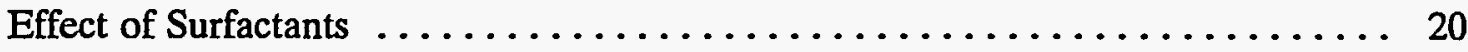

Stability of the Extractant $\ldots \ldots \ldots \ldots \ldots \ldots \ldots \ldots \ldots \ldots \ldots \ldots \ldots \ldots \ldots \ldots \ldots \ldots, 26$

Effect of Ultrasonic Frequency $\ldots \ldots \ldots \ldots \ldots \ldots \ldots \ldots \ldots \ldots \ldots \ldots \ldots \ldots \ldots \ldots, 27$

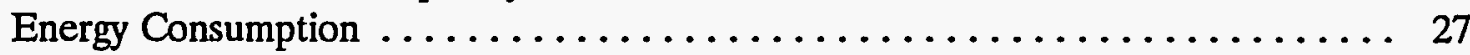

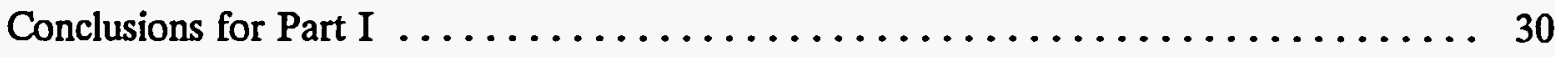


PART II: MECHANISMS ASSOCIATED WITH THE SOLVENT EXTRACTION OF GALLIUM USING KELEX 100 EXTRACTANT $\ldots \ldots \ldots \ldots \ldots \ldots \ldots \ldots \ldots, 31$

Introduction $\ldots \ldots \ldots \ldots \ldots \ldots \ldots \ldots \ldots \ldots \ldots \ldots \ldots \ldots \ldots \ldots \ldots \ldots \ldots \ldots$

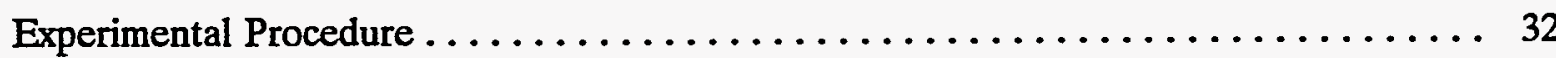

Results and Discussion $\ldots \ldots \ldots \ldots \ldots \ldots \ldots \ldots \ldots \ldots \ldots \ldots \ldots \ldots \ldots \ldots \ldots$

Effect of Stirring Speed $\ldots \ldots \ldots \ldots \ldots \ldots \ldots \ldots \ldots \ldots \ldots \ldots \ldots \ldots \ldots \ldots \ldots \ldots$

Effect of Temperature $\ldots \ldots \ldots \ldots \ldots \ldots \ldots \ldots \ldots \ldots \ldots \ldots \ldots \ldots, 33$

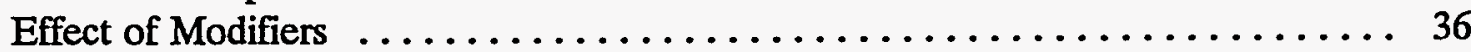

Effect of Aqueous Solution Composition ..................... 36

Extraction Mechanism $\ldots \ldots \ldots \ldots \ldots \ldots \ldots \ldots \ldots \ldots \ldots \ldots \ldots, 41$

Conclusions for Part II ............................... 47

PART III: THE EFFECT OF ULTRASOUND ON THE SOLVENT EXTRACTION OF

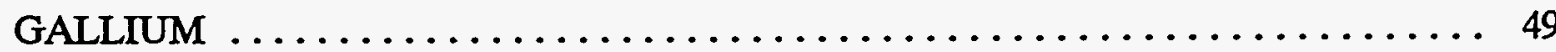

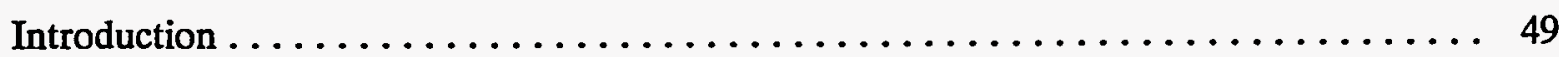

Experimental Procedure $. \ldots \ldots \ldots \ldots \ldots \ldots \ldots \ldots \ldots \ldots \ldots \ldots . \ldots . \ldots$

Sonochemical Extraction of Gallium from Synthetic Sodium Aluminate Solutions ..... 50

Effect of Stirring Speed $\ldots \ldots \ldots \ldots \ldots \ldots \ldots \ldots \ldots \ldots \ldots \ldots \ldots \ldots$

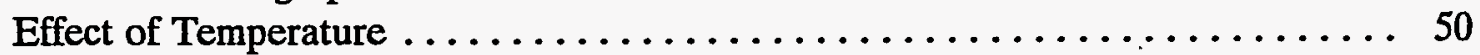

Effect of Diluents and Modifiers ......................... 53

Effect of Sodium Hydroxide and Aluminum Hydroxide Concentration ........ 56

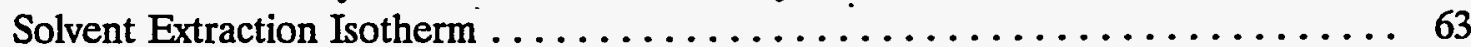

Sonochemical Extraction of Gallium from an Industrial Process Solution $\ldots \ldots \ldots \ldots 63$

Effect of Diluents $\ldots \ldots \ldots \ldots \ldots \ldots \ldots \ldots \ldots \ldots \ldots \ldots \ldots \ldots \ldots \ldots \ldots \ldots \ldots, 63$

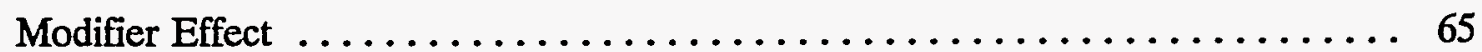

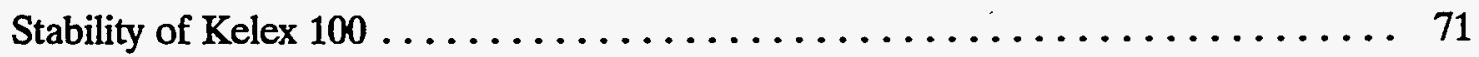

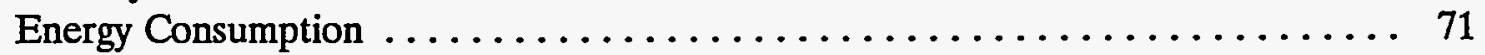

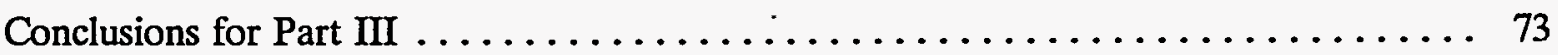

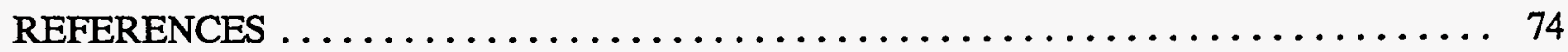




\section{FIGURES}

1. The effect of stirring speed on the solvent extraction of nickel with Lix $65 \mathrm{~N}$ - lauric acid - Kermac 470B and Lix 70 - lauric acid - Kermac 470B

2. The effect of the initial $\mathrm{pH}$ on nickel extraction with Lix $65 \mathrm{~N}$ - lauric acid -

Kermac 470B .

3. The effect of the initial $\mathrm{pH}$ on nickel extraction with $\mathrm{Lix} 70$ - lauric acid -

Kermac 470B ................................... 14

4. The effect of temperature on nickel extraction with Lix $65 \mathrm{~N}$ - lauric acid -

Kermac 470B ..................................... 16

5. The effect of temperature on nickel extraction with Lix 70 - lauric acid - Kermac 470B - 17

6. The effect of initial nickel concentration on nickel extraction with Lix 70 - lauric acid Kermac 470B ... . . . . . . . . . . . . . . . . . . . . . . . . . . . . . . . 19

7. Extraction isotherm of nickel with $\operatorname{Lix} 65 \mathrm{~N}$ - lauric acid - Kermac $470 \mathrm{~B} \ldots \ldots \ldots \ldots$

8. Extraction isotherm of nickel with Lix 70 - lauric acid - Kermac $470 \mathrm{~B} \ldots \ldots \ldots \ldots$

9. The effect of ultrasound on the solvent extraction of Cobalt with $\mathrm{Lix} 70$ - lauric acid Kermac 470B at $\mathrm{pH} 5$

10. The extraction of nickel and Co with Lix 70 - lauric acid - Kermac 470B in the absence of ultrasound

11. The extraction of nickel and Co with $\mathrm{Lix} 70$ - lauric acid - Kermac 470B in the presence of ultrasound

12. The effect of temperature on the solvent extraction of gallium with Kelex 100 - decanol - Kermac 470B

13. An Arrhenius plot for the solvent extraction of gallium with Kelex 100 - decanol Kermac 470B

14. The effect of modifiers on the solvent extraction of gallium with Kelex 100 and Kermac 470B

15. The extraction of gallium as a function of sodium hydroxide concentration

16. The effect of alkali concentration on the apparent forward reaction rate constant, $\mathbf{k}_{\mathrm{f}}$, for the extraction of gallium with Kelex 100 - decanol - Kermac 470B 
17. The effect of alkali concentration on the apparent forward reaction rate constant, $\mathbf{k}_{\mathrm{f}}$, for the extraction of gallium with Kelex 100 - 2-undecanone - Aromatic $150 \ldots \ldots .40$

18. The extraction of sodium into the organic phase as a function of the initial aqueous sodium hydroxide concentration during the solvent extraction of gallium $\ldots \ldots \ldots \ldots 42$

19. Solvent extraction of gallium as a function of alumina concentration $\ldots \ldots \ldots \ldots \ldots 43$

20. NMR spectra of ${ }^{71} \mathrm{Ga}$ and ${ }^{27} \mathrm{Al}$ for the aqueous solutions $\ldots \ldots \ldots \ldots \ldots \ldots$

21. The effect of stirring speed on the solvent extraction of gallium with Kelex 100 -

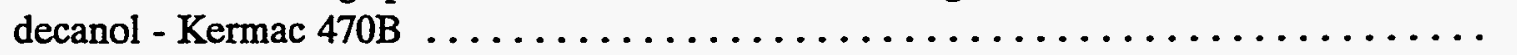

22. The effect of temperature on the solvent extraction of gallium with Kelex 100 - decanol - Kermac 470B ..................................... 52

23. The extraction of gallium in the presence of ultrasound using four different diluents and decanol as a modifier

24. The extraction of gallium in the absence of ultrasound using four different diluents and decanol as a modifier.

25. The extraction of gallium in the absence of ultrasound using four different diluents and 2-undecanone as a modifier

26. The extraction of gallium in the presence of ultrasound using four different diluents and 2 -undecanone as a modifier $\ldots \ldots \ldots \ldots \ldots \ldots \ldots \ldots \ldots \ldots \ldots \ldots \ldots$

27. The effect of sodium hydroxide concentration on the extraction of gallium in the

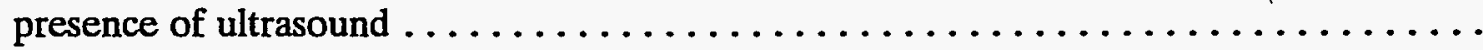

28. The effect of alumina concentration on the solvent extraction of gallium in the absence of ultrasound ....

29. The effect of alumina concentration on the solvent extraction of gallium in the

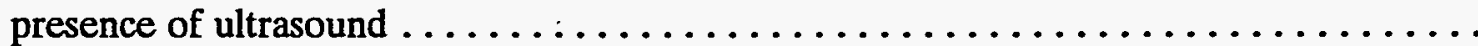

30. Extraction isotherm of gallium with Kelex 100 - decanol - Kermac 470B ........ 64

31. The effect of diluents on the extraction of gallium from an industrial process solution in the absence of ultrasound

32. The effect of diluents on the extraction of gallium from an industrial process solution in

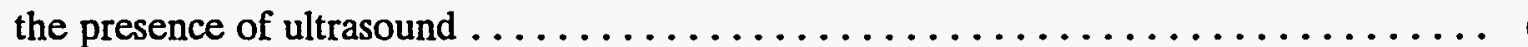

33. The effect of modifiers with Kermac 470B on the extraction of gallium from an industrial process solution in the presence of ultrasound 
34. The effect of modifiers with Aromatic 150 on the extraction of gallium from an industrial process solution in the presence of ultrasound $\ldots \ldots \ldots \ldots \ldots \ldots \ldots$

35. The effect of modifiers with Escaid 200 on the extraction of gallium from an industrial process solution in the presence of ultrasound $\ldots \ldots \ldots \ldots \ldots \ldots \ldots \ldots \ldots \ldots$

36. Energy consumption as a function of the mode of intermittent ultrasonic action for the solvent extraction of gallium from a synthetic sodium hydroxide solution

\section{TABLES}

1. The effect of the initial $\mathrm{pH}$ on solvent extraction of nickel in the absence and in the

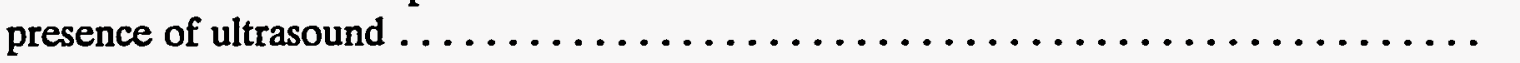

2. Effect of temperature on solvent extraction of nickel in the absence and in the

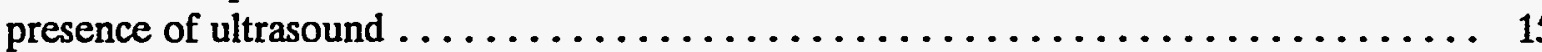

3. Activation energy for nickel extraction in the absence and in the presence of

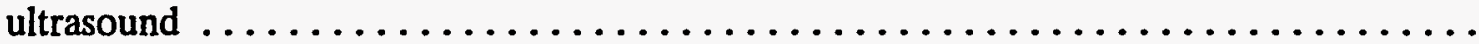

4. Comparison of the effect of DNNSA as a surfactant and ultrasound on the time to

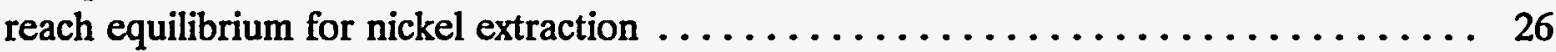

5. Multiple cycle test for extraction of nickel with $\mathrm{Lix} 65 \mathrm{~N}$ - lauric acid - Kermac 470B _.. 27

6. Ultrasonic energy consumption during nickel extraction with Lix $65 \mathrm{~N}$; ultrasound was used in intermittent mode $\ldots \ldots \ldots \ldots \ldots \ldots \ldots \ldots \ldots \ldots \ldots \ldots \ldots, 28$

7. Ultrasonic energy consumption during solvent extraction of nickel by using a continuous flow of organic and aqueous phases

8. Effect of temperature on the apparent forward reaction rate constant, $\mathrm{k}_{\mathrm{f}}$, during $\mathrm{Ga}$

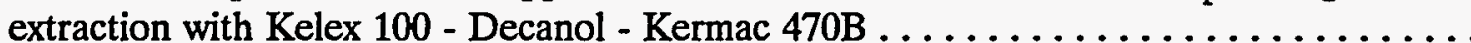

9. Apparent forward reaction rate constant, $\mathrm{k}_{\mathrm{f}}$ for the solvent extraction of gallium in the presence and in the absence of ultrasound (data from Figure 22$) \ldots \ldots \ldots \ldots \ldots$

10. The effect of diluents on the apparent forward reaction rate constant, $\mathrm{k}_{\mathrm{f}}$ for the solvent extraction of gallium in the presence and the absence of ultrasound using decanol as a modifier (data from Figures 23 and 24)

11. The effect of diluents on the apparent forward reaction rate constant, $\mathrm{k}_{\mathrm{f}}$, for solvent extraction of gallium in the presence and in the absence of ultrasound using 2-undecanone as a modifier (data from Figures 25 and 26)

12. The effect of sodium hydroxide concentration on the extraction rates of gallium in the absence and in the presence of ultrasound (data taken from Figure 16 and Figure 27) . . 
13. The effect of aluminum hydroxide concentration on the extraction rates of gallium in the absence and in the presence of ultrasound (data taken from Figures 28 and 29) ... .

14. The composition of the industrial Bayer process leach liquor (evaporator discharge) from Kaiser Aluminum Company

15. The effect of diluents on the apparent forward reaction rate constant, $k_{\mathfrak{f}}$ for the solvent extraction of gallium from an industrial process solution in the presence and in the absence of ultrasound (data from Figures 31 and 32 ) $\ldots \ldots \ldots \ldots \ldots \ldots \ldots \ldots$

16. The effect of modifiers on the rate of extraction of gallium from Bayer process leach liquor in the presence of ultrasound 


\section{Application of Ultrasound in Solvent Extraction of Nickel and Gallium}

\section{INTRODUCTION}

The objective of the research described in this report was to investigate the potential benefits of the use of ultrasound in solvent extraction processes. Two metal extractions were selected specifically for their sluggish nature: the solvent extraction of nickel from a synthetic aqueous leach solution, and the solvent extraction of gallium from synthetic and industrial sodium aluminate solutions. Neither nickel nor gallium are currently recovered in the United States. These metals could be recovered by solvent extraction processes; however, the rate of extraction is notably slow in both cases. The use of ultrasound to significantly enhance the rates could make the solvent recovery processes more economical.

After a short background section that provides a review of the use of ultrasound in metallurgical processes, this report is divided into three parts. The results of a study of the effect of ultrasound on the solvent extraction of nickel from synthetic aqueous leach solutions using two different hydroxyoxime extractant systems are presented in Part I. The results of a study of the solvent extraction of gallium is then presented in Parts II and III of this report. The mechanisms involved during the normal solvent extraction of gallium without ultrasound was studied first, and these results are presented in Part II. The effect of ultrasound on the solvent extraction of gallium was then investigated, and these results are presented in Part III. 


\section{BACKGROUND}

Ultrasound is an acoustic pressure wave having a vibrational frequency above the level detectable by the human ear $(>20 \mathrm{kHz})$. Ultrasound was discovered at the end of the last century. Ultrasonic energy can be transmitted through any substance (solid, liquid, and gas), and can variously influence the medium through which it passes. Physicists were first interested in the study of ultrasound as a novel method for energy transmission. The study of ultrasonic energy transmission in liquids soon led to the discovery of a cavitation bubble phenomenon and a cavitation effect (bubble formation and implosion). Although this energy dissipation mechanism is an impediment for energy transmission, it was found to have useful applications in chemistry, chemical engineering, and metallurgy where it can influence various chemical reactions. The use of ultrasound to modify chemical reactions is called "sonochemistry."

The systematic study of sonochemistry began in $1927 .^{1}$ It was found that ultrasonic energy could be used to break chemical bonds and initiate new reactions. Some of the most interesting applications for chemists were cavitation-induced redox reactions in aqueous solutions; the reaction of organic molecules, such as hydrolysis of dimethyl sulfate; and the use of ultrasound in the preparation of various organic chemicals. Researchers found that the use of ultrasound significantly accelerated the rate of these chemical reactions.

Chemical engineering processes that were found to benefit from the use of ultrasound include crystallization, filtration, extraction, emulsification, coagulation, degassing, and atomization processes. These operations occur widely in homogeneous and heterogeneous phase mixtures (solid-gas, liquid-gas, solid-liquid, and liquid-liquid).

Extraction metallurgists have interests in the application of ultrasound to processes such as leaching, flotation, ion exchange, electrowinning, and solvent extraction. Most studies of the application of ultrasound to metallurgical processes have been performed on solid-liquid or liquidliquid systems.

For solid-liquid systems, the application of ultrasound, with associated induced cavitation in the liquid phase, apparently improves reaction rates by increasing the interphase mass and heat transfer rates. Bubble cavity implosions create short-lived temperature increases as high as $5500^{\circ} \mathrm{C}$ for gaseous contents inside the bubble, and as high as $2100^{\circ} \mathrm{C}$ for the immediate surrounding liquid. The implosions also create short-lived peak pressures as high as 500 atm. ${ }^{2}$ When the ultrasonic energy interacts with a solid surface, the cavity implosions near the liquidsolid interface not only disturb the stagnant layer between the solid and the liquid, but also remove the protective coating on the surface of the solid. The latter effect, caused by a jet of liquid directed at the solid, is generated by an asymmetric implosion of the cavity. The jet also degasses the surface of the solid and changes its character to make the liquid more accessible for chemical reactions to occur.

Acoustic streaming is a phenomenon, independent of cavitation, that also promotes increased mass transfer in solid-liquid systems. As a liquid absorbs energy from a propagating acoustic wave, it acquires corresponding momentum, thus creating force gradients and mass transfer. Such streaming will occur at moving solid surfaces or at vibrating bubbles. Both cavitation and acoustic streaming effects may happen simultaneously. It is believed that these 
mechanisms are responsible for the rate enhancement of processes such as ore flotation, leaching, adsorption, and electrodeposition.

With immiscible liquid-liquid systems, such as oil and water, another effect of the use of ultrasound can occur. Research has indicated that, due to the compression and expansion of the liquid media by the ultrasound, larger liquid droplets can be torn into smaller parts and the total interfacial surface area between the two immiscible liquids can be increased. Eventually, an emulsion can be formed. Emulsification can significantly accelerate mass transfer and heterogeneous chemical reaction rates. Local agitation, induced circulation currents and prolateoblate oscillations inside the liquid droplet, and the removal of the stagnant liquid in the vicinity of the interface can also result in positive effects. It is believed that these effects are responsible for increasing the rate of liquid-liquid processes, such as solvent extraction.

In general, the effects of ultrasound are strongly influenced by a number of external parameters including the properties of the ambient gas and liquid, static pressure, bulk temperature, acoustic intensity, and acoustic frequency. Cavitation produces bubbles of gas, and the sonochemical effects depend on the nature of the gas and the vapor pressure of the solvent. The use of a solvent with a high vapor pressure will result in less intense cavitational effects. An increase in bulk temperature can decrease the ultrasonic effect by increasing the vapor pressure. Solvent and dissolved gas with the lowest thermal conductivity will generally produce the most efficient cavitation. An increase in acoustic intensity has been recognized to result in increased sonochemical effects, but an increase in acoustic frequency generally results in a greater power requirement to produce cavitation in a liquid. (For some systems; however, high frequency ultrasound may provide a method to quickly break down emulsions after the extraction reaction has been achieved.)

The availability of equipment for producing ultrasound has been the critical factor limiting increased application of the technique. In 1927, Richards and Loomis ${ }^{1}$ had used quartz crystals immersed in oil between electrodes to produce ultrasound (100 to $500 \mathrm{kHz}$ ). Further research was not performed until the early $1950 \mathrm{~s}$, when suitable equipment became available for general use as a result of the technology developed for sonar detection during World War II. However, even then, because of a lack of understanding of the fundamental properties of ultrasound, and because of the relatively poor quality and reliability of the equipment, most of the projects failed in the pilot scale. ${ }^{3}$ In the 1960 s, a cobalt barium titanate transducer was introduced. It produced vibrations in the higher region of the ultrasonic spectrum (500 to $1000 \mathrm{kHz}$ ). ${ }^{4}$ However, ultrasonic research was deferred until the 1980s. Renewed interest stemmed from the marketing of lead zirconate titanate (PZT) ceramics for generators in the frequency range of 15 to $50 \mathrm{kHz}$, and from the development of direct immersion titanium alloy horns.

The search for suitable commercial equipment for ultrasonic energy generation continues. Future applications of the use of ultrasound in metallurgical processes will depend on the development of inexpensive, reliable, and energy efficient large-scale generation equipment.

\section{Ore Beneficiation}

The use of ultrasound to selectively remove absorbed layers of reagents from minerals during the flotation process has been found to be a useful technique in ore beneficiation. For sulfide 
minerals it was reported that, when used with the flotation process, ultrasound destroys the adsorbed reagent layer on pyrite completely, while leaving the chalcopyrite almost unaffected. Thus with the use of ultrasound, pyrite and chalcopyrite can be separated by this process. ${ }^{5}$

Alteration of the mineral surface properties by ultrasound before beneficiation is another technique. Ultrasonic treatment increases the number of active points on a crystal surface as a result of increased dislocations. The method was found effective for changing the surface properties of particles and for increasing the selectivity of flotation, electrostatic, and magnetic beneficiation. Using ultrasound, Akopova et al. ${ }^{6}$ increased $\mathrm{Ti}$ and $\mathrm{Zr}$ mineral recovery using tall oil in the flotation process. The minerals were treated with ultrasound $(22 \mathrm{kHz}$ frequency and $3 \mathrm{~W} / \mathrm{cm}^{2}$ intensity), at $20^{\circ} \mathrm{C}$ and a solid/liquid ratio of $1: 10$. After one minute of ultrasonic treatment, zircon recovery increased from $7.7 \%$ to $53.7 \%$, rutile recovery increased from $1.4 \%$ to 98\%, and staurolite recovery increased from $1.7 \%$ to $98 \%$.

In addition to improving the rate of extraction or the extraction yield of minerals, ultrasonic treatment can result in reduced consumption of reagents and enable the use of less expensive reagents. Ultrasound can be used to emulsify flotation reagents, which are often difficult to dissolve. 7,8 Very stable emulsions can be produced using ultrasound alone without the need for additional stabilizing reagents.

\section{Leaching}

Ultrasonic vibrations can intensify the dissolution rates of various minerals. The greatest effect is found in cases in which the process reaction rate is limited by diffusion, especially when this process is accompanied by the formation of solid reaction products on the surface of the phase contacts. Such conditions occur in the acid leaching of tungsten concentrates and in the sulfuric acid treatment of calcium-bearing ores, for example. The action of ultrasound in speeding up these processes is due to the cavitational destruction of the surface layers on the solid particles and to the micro-turbulent pulsations that influence the state of the boundary layers in the liquid phase.

Ultrasound can be used to accelerate the leaching of tungsten from scheelite either with $\mathrm{Na}_{2} \mathrm{CO}_{3}$ in an autoclave or by decomposition with $\mathrm{HNO}_{3}{ }^{9}$ In the autoclave leaching process, tungsten extraction was increased from $-10 \%$ to $\sim 87 \%$. The process pressure was also decreased. (These results were reported for a $2.5 \mathrm{~h}$ leach at a soda equivalent of 1.6 to 1.8.) For the leaching of scheelite concentrate with $\mathrm{HNO}_{3}$, the dissolution changed from a diffusion rate controlled process (which is due to the formation of a hydrated film of $\mathrm{H}_{2} \mathrm{WO}_{4}$ using mechanical stirring alone), to a kinetic rate controlled process with ultrasound. With an excess of a $30 \%$ $\mathrm{HNO}_{3}$ solution, the use of ultrasound enabled $99 \%$ of scheelite to be dissolved after $1 \mathrm{~h}$ at $90^{\circ} \mathrm{C}$. This result can be compared to a typical time of $\sim 25 \mathrm{~h}$ to achieve similar dissolution levels without ultrasound.

Another study investigated the effect of ultrasound on the sulfuric acid leaching of roasted zinc concentrates. ${ }^{10}$ Ultrasound was found to be most effective at increasing the rate of dissolution of oxidized zinc, a process that is normally diffusion rate controlled. Ultrasound had only a small effect on the dissolution of ferrite zinc, which is normally kinetic rate controlled without the use of ultrasound. 
In a study of the effect of ultrasound $\left(18.8 \mathrm{kHz}, 2.8 \mathrm{~W} / \mathrm{cm}^{2}\right)$ on the leaching of copper from chrysocolla, chalcocite, and chalcopyrite (solid to liquid ratio of 1:5), it was found that leaching was intensified by a factor of two to three. ${ }^{11}$ The best leaching medium was $5 \% \mathrm{H}_{2} \mathrm{SO}_{4}$ for chrysocolla and ferric sulphate, and $2 \% \mathrm{H}_{2} \mathrm{SO}_{4}$ for chalcocite and chalcopyrite. In $30 \mathrm{~min}$ at $T=50^{\circ} \mathrm{C}, 99.8 \%, 71.2 \%$, and $3 \%$ of the $\mathrm{Cu}$ was leached from chrysocolla, chalcocite, and chalcopyrite, respectively. Corresponding values in the absence of ultrasound were $50.6 \%, 46.9 \%$, and $0 \%$. In another example, when nickel and copper were leached in $\mathrm{H}_{2} \mathrm{SO}_{4}$ at $T=30^{\circ} \mathrm{C}$, Konyaev ${ }^{12}$ reported an increase in the rate of dissolution of nickel by a factor of eight, and an increase in the rate of dissolution of copper by a factor of three under the influence of ultrasound $\left(23 \mathrm{kHz}, 1\right.$ to $1.2 \mathrm{~W} / \mathrm{cm}^{2}$ ).

In one study of the ultrasonic treatment of flotation concentrates containing 1.4 to $2.3 \mathrm{oz}$ $\mathrm{Au} / \mathrm{ton}$, the time required for cyanidation decreased by a factor of two to three, and the gold extraction simultaneously increased. ${ }^{13}$ In another study, Glukhov and Pronishchev ${ }^{14}$ found that the cyanidation rate of gold-containing flotation concentrates was increased by a factor 2 to 12 when ultrasound was used ( 4 to $5 \mathrm{~W} / \mathrm{cm}^{2}$ and $21.3 \mathrm{kHz}$ ). The recovery of gold also increased, depending on its dispersion and its association with other minerals.

In an effort to develop high capacity, low cost acoustic sources that could be used to improve the leaching of gold and silver-containing ore, hydrodynamic converters were developed that operate on the principle of converting the energy from a stream of liquid into elastic oscillation energy. Gold and silver extraction increased when a Rotor-Impulse device was used, and cyanidation time was shortened by 6 hours. ${ }^{15}$

In a recent preliminary test, a production-scale Bodine Sonic Oscillator device $(60-80 \mathrm{~Hz}$, electrically-powered) was used to leach gold and silver concentrates (from eastern Nevada) at Metex Corporation in Midvale, Utah. ${ }^{16}$ The results of the test showed that $86 \%$ of the gold and $39 \%$ of the silver could be extracted in 90 minutes with the use of the acoustic device. These results can be compared to $96 \%$ of the gold and $37 \%$ of the silver leached in 33 hours with a normal (no sonic energy) process. In addition, the cyanide consumption may have been significantly less than a normal leach $(4.0 \mathrm{lb} / \mathrm{ton})$.

\section{Electrometallurgy}

Perhaps the most significant benefits from the application of ultrasound can be found in the field of electrometallurgy; an excellent review is given by Walker. ${ }^{17}$

Beneficial results have been obtained from the application of ultrasound in electrowinning, electroplating, and electrorefining. These benefits include a large increase in current density, and a simultaneous decrease in the polarization voltage, with the complete elimination of the diffusion layer at the electrodes. Removal of any gaseous phase near the electrodes by ultrasonics is another beneficial effect, and smooth, hard deposits can be produced using ultrasound, without the need for additional agents that could be codeposited during electrodeposition. ${ }^{18}$

In the presence of ultrasound $\left(30 \mathrm{kHz}, 0.25 \mathrm{~W} / \mathrm{cm}^{2}\right)$, the current efficiency for copper electrowinning with a cyanide bath was significantly increased from $53 \%$ to $86 \%$, and the voltage 
was reduced from $2.93 \mathrm{~V}$ to $1.83 \mathrm{~V}$, at a current density of $4 \mathrm{~A} / \mathrm{dm}^{2} .19$ Similar results have been obtained for other metals $(\mathrm{Ag}, \mathrm{Cr}, \mathrm{Ni}, \mathrm{Cd}$, and $\mathrm{Zn})$. However, the use of ultrasound produced pitted $\mathrm{Zn}$ deposits at all current densities.

A study of the electrorefining of copper showed that, with ultrasound $(22 \mathrm{kHz}$ and $1.5 \mathrm{~W} / \mathrm{cm}^{2}$ ) in a solution containing $45 \mathrm{~g} / \mathrm{L}$ copper, $150 \mathrm{~g} / \mathrm{L}$ sulfuric acid, and $0.5 \mathrm{~g} / \mathrm{L}$ glue at 30 to $40^{\circ} \mathrm{C}$, the voltage drop between the electrodes was reduced from $6.6 \mathrm{~V}$ to $2.0 \mathrm{~V}$ at a current density of $20 \mathrm{~A} / \mathrm{dm}^{2} .20$ The consumption of electrical power was reduced from more than 3890 $\mathrm{kW} \cdot \mathrm{h} /$ ton of copper produced to less than $1670 \mathrm{~kW} \cdot \mathrm{h} / \mathrm{ton}$. When the frequency of the ultrasound was increased from $22 \mathrm{kHz}$ to the range 400 to $800 \mathrm{kHz}$, less satisfactory results were obtained.

Eggett et al. ${ }^{21}$ considered the possibility of using ultrasound to assist in the electrowinning of copper at high current densities on a large scale. It was determined that the most satisfactory method of introducing ultrasonic energy was to locate probes directly in the floor or walls of the electrochemical cell. With this system and an ultrasonic power of $200 \mathrm{~W}\left(97 \mathrm{~W} / \mathrm{dm}^{2}\right.$ of electrolyte plan area), the operating cell voltage was reduced $\sim 50 \mathrm{~V}$ throughout the range of current density tested. The quality of the copper deposits produced with the ultrasonic agitation was considered good. Although the total power requirements increased with the use of ultrasound, a significant savings in total capital cost could still result for a 100 ton/d tankhouse.

\section{Ion Exchange}

Studies of the effects of ultrasound on ion exchange rates are somewhat inconclusive. Although some researchers report beneficial results, others do not agree.

Shostak et al. ${ }^{22}$ studied the static ion-exchange capacities of KU-21 (a strong acid cation exchanger) and of EDE-10P and $\mathrm{AN}-1$ (medium and low-base anion exchangers) in the presence of ultrasound $\left(3.0 \mathrm{~W} / \mathrm{cm}^{2}\right)$, and they reported that the ultrasonic agitation was more effective than mechanical stirring. The positive effect depends on properties of the ion exchanger and on solution concentration. When EDE-10P was used in $1 \mathrm{~N} \mathrm{HCl}$, the rate of exchange in the presence of ultrasound was doubled; in $0.1 \mathrm{~N} \mathrm{HCl}$, it was quadrupled.

Khavskii et al. ${ }^{23}$ observed a two-fold increase in the molybdenum sorption rate with the SG1 ion exchanger when ultrasound was applied, and the resin capacity was simultaneously increased by 15 to $20 \%$. In another literature reference, Khavskii et al. ${ }^{24}$ claimed that the effect of ultrasound is not limited to the surface layers of the resin particles. Secondary phenomena occurring during the cavitation leads to complete exposure of micropores in the sorbent particles. At the same time, the cavitation induced pressure pulsations can facilitate the penetration of solutions into the relatively inaccessible microcapillaries in the sorbent particles.

However, Chang and Wang, ${ }^{25}$ after careful experimentation with strong acidic and basic resins, reported contradictory results on the importance of ultrasound on ion exchange rates. They claimed that energy of the shockwave that occurs when the cavitation bubble collapses would interrupt film diffusion of the ions. 
Ultrasound may find applications in ion-exchange chromatographic separations. Recently, according to a Japanese patent, ${ }^{26}$ the ultrasonic waves $\left(100 \mathrm{kHz}, 2 \mathrm{~W} / \mathrm{cm}^{2}\right)$ applied together with a magnetic field accelerated the separation of mixed rare earths from bulk $\mathrm{La}$ and $\mathrm{Ce}$ through a strongly acidic cation-exchange column (10-m length in 30 columns). A Yttrium purity of $99.3 \%$ was achieved in a 30-h cycle, which can be contrasted with a 240-h cycle needed to attain a 99.94-Y purity level in the absence of ultrasound and a magnetic field.

Numerous applications of the use of ultrasound in the ion exchange desorption processes for waste water treatment applications have been reported. ${ }^{27}$

\section{Absorption}

Significant increases in the loading rates of $\mathrm{Au}$ and $\mathrm{Ag}$ solutions on a carbon column were reported. ${ }^{28}$ Similar enhanced loading rates were expected to be achieved for carbon-in-pulp and carbon-in-leach circuits.

\section{Solvent Extraction}

Most of the research dealing with solvent extraction was performed on systems in which the interphase mass transfer occurred without chemical reactions. Woodle and Vilbrandt ${ }^{29}$ studied the mechanism of ultrasonic action during the transfer of methyl alcohol into toluene. The positive effect of ultrasound was ascribed to the production of a larger interfacial surface area, the removal of a stagnant liquid layer, and the increased circulation within droplets. Enhanced extraction rates in the presence of ultrasound were also observed by Chen and Chon, ${ }^{30}$ and Flisak and Perna, ${ }^{31}$ who studied the extraction of acetic acid with methyl isobutyl ketone.

Solvent extraction systems in which a chemical reaction step was present were studied by Vasil'ev and Kaveilya. ${ }^{32}$ They reported a significant increase in the extraction rates of $\mathrm{Co}, \mathrm{Cu}$, $\mathrm{Zn}$, and $\mathrm{Bi}$ with dithiozone $-\mathrm{CCl}_{4}$ in the presence of ultrasound.

Khavskii, Yakubovich, and Tokarev ${ }^{33}$ studied the cavitation effects on the micellar structure of quaternary ammonium salts. An increased conductivity of the organic phase was explained by the breakdown of the micellar aggregates of the amines, aa phenomenon that increased their extractive capacity.

Ultrasound can be used to form emulsions to increase the rate of solvent extraction by increasing the interfacial surface area. Ultrasound can also be used to break emulsions after solvent extraction in order to allow the phases to settle. Khavskii et al. ${ }^{34}$ reported a study related to this latter use of ultrasound. An emulsion was formed by contacting sodium tungstate and sodium molybdate (10 $\mathrm{g} / \mathrm{L}$ Mo and $100 \mathrm{~g} / \mathrm{L} \mathrm{W})$ with TBP (tributyl phosphate) at an aqueous phase to organic phase ratio of $1: 1$ by volume $(\mathrm{A} / \mathrm{O}=1: 1)$, and a $\mathrm{pH} \sim 1$. This emulsion was subjected to ultrasound $\left(1 \mathrm{MHz}, 0.2 \mathrm{~W} / \mathrm{cm}^{2}\right)$ for 15 minutes and then allowed to settle for 4 to 8 minutes. It was found that the separation of the emulsion was 4 to 5 times faster with the ultrasound treatment than without it. It was also found that, if the frequency of ultrasound was less (for example $21.8 \mathrm{kHz}$ ), no improvement in the rate of separation of the emulsion was obtained. 
The ability of ultrasound to assist in the separation of emulsions proceeds in two stages. ${ }^{35}$ In the first stage, coagulation of the emulsion particles is promoted by the ultrasonic pressure and by orthokinetic collisions. In the second stage, which occurs slowly, the particles coagulate by hydrodynamic forces. The nature of the dispersed system plays an important role in the separation process. 


\section{PART I: THE EFFECT OF ULTRASOUND ON THE SOLVENT EXTRACTION OF NICKEL}

\section{Introduction}

Most hydrometallurgical processes are heterogeneous in nature involving two distinct phases: solid-liquid or liquid-liquid. The use of ultrasound has been shown to provide beneficial influences in processes such as leaching, adsorption, and electrodeposition, which involve interactions at the solid-liquid interface. $17,24,28,36,37$ It is believed that the cavitation (bubble formation and implosion), microstreaming, acoustic pressure, and degassing are effects responsible for the positive role of ultrasound in these systems.

Solvent extraction is a hydrometallurgical process that involves two distinct and immiscible liquid phases, usually one aqueous and one organic phase. The use of ultrasound can increase the surface area between the two liquid phases (possibly even producing an emulsion), resulting in a significant increase in the overall extraction rate. Other beneficial effects of the use of ultrasound in solvent extraction systems can be the result of enhanced local agitation, induced circulation currents and prolate-oblate oscillations inside the liquid drop, and the removal of the stagnant liquid in the vicinity of the interface between organic and aqueous phases. ${ }^{29}$

Most of the studies of the effects of ultrasound on solvent extraction systems have been performed on systems that do not involve chemical reactions. ${ }^{29,30,31}$ However, from the hydrometallurgical point of view, a large number of metal solvent extraction systems are controlled by chemical reaction mechanisms for which the effect of ultrasound should be examined. One solvent extraction system that is notably slow is the extraction of nickel from an aqueous leach solution. The effect of ultrasound on the solvent extraction of nickel using a hydroxyoxime extractant is considered here.

The hydroxyoxime and carboxylic acid system for the selective extraction of nickel from cobalt in acidic sulfate solutions was first recommended by Flett and West. ${ }^{38}$ They found that when carboxylic acid was added to the $\alpha$-hydroxyoxime, Lix 63 , a synergistic effect occurred, increasing the yield of nickel extraction significantly. But the rate of extraction was so slow that it took $\sim 3 \mathrm{~h}$ to reach equilibrium. From interfacial tension measurements it was determined that the carboxylic acid was present at the interface between the organic and aqueous phase. $39,40,41$ However, because of the complicated extraction mechanism involved in the reaction, the reasons for the slow nickel extraction rates were not explained.

A number of ways were suggested to increase the extraction rate of nickel. Nyman and Hummelsedt ${ }^{42}$ reported that temperature and the type of diluent considerably influenced the extraction rate. They suggested that the addition of a dinonylnaphthalene sulfonic acid surfactant (DNNSA) to the Lix $65 \mathrm{~N}$ - Versatic 911 or the Lix 70 - Versatic 911 systems could accelerate the nickel extraction rate. In another reference, Hummelstedt et al. ${ }^{43}$ studied the Kelex 100 Versatic 911 system and found that the nickel extraction rate was also very slow in this system. A temperature increase was recommended to increase the rate of extraction. Flett, Cox, and Heels ${ }^{44}$ showed that the addition of Versatic 911 to Kelex 100 results in an interaction between these two extractants such that the oxidation and extraction of Co (II) can be prevented. 


\section{Experimental Procedure}

The hydroxyoxime extractants used in this study, Lix $65 \mathrm{~N}$ and $\mathrm{Lix} 70$, were provided by Henkel Corp. (Lot Nos. 82B265010 and 8J16029, respectively). Lauric acid (a carboxylic acid) was provided by Eastman Kodak Co. The surfactant, dinonylnaphthalene sulfonic acid (DNNSA), was provided by Pfaltz \& Bauer Inc. Other chemicals included the diluent Kermac 470B (Triangle Refineries Co.), $\mathrm{NiSO}_{4} 6 \mathrm{H}_{2} \mathrm{O}$, and $\mathrm{KNO}_{3}$, analytical reagent grade (A.R.).

A specially designed, vigorously stirred, baffled reactor $71 \mathrm{~mm} \mathrm{ID} \mathrm{and} 85 \mathrm{~mm}$ high was used. The reactor was equipped with a turbine-type Teflon stirrer $25 \mathrm{~mm}$ in diameter and $5.5 \mathrm{~mm}$ in height. Two Teflon baffles, each $13 \times 80 \mathrm{~mm}$, were mounted near the wall of the reactor.

The reactor cover was designed to accept an ultrasonic horn, a mechanical stirrer, and a sampler. Ultrasound was produced with a Branson Model 184-V generator and a Branson Model 102 convertor. A 1-in. diameter titanium horn was used to transmit the ultrasonic energy into the solvent extraction system. The frequency of the ultrasound was $20 \mathrm{kHz}$. The standard ultrasonic power input was $47 \mathrm{~W}$. To avoid a temperature increase in the reactor as a result of the dissipation of the ultrasonic energy, the reactor had to be submerged in a water bath. The water in the bath was cooled by a refrigeration unit supplied with a cooling coil.

The organic and aqueous (nickel-free) phases were first transferred into the reactor, and the ultrasound was turned on. After an equilibrium temperature was reached, the experiment began by adding $1 \mathrm{~mL}$ of nickel solution to the aqueous phase. Samples were taken at specific time intervals and centrifuged immediately. The extent of the solvent extraction of nickel from the aqueous phase to the organic phase was determined by analyzing the nickel concentration remaining in the aqueous phase using an atomic absorption spectrophotometer.

In the solvent extraction system, the organic phase consisted of $10 \%(\mathrm{v} / \mathrm{v}) \mathrm{Lix} 65 \mathrm{~N}$ (or Lix 70), $0.1 M$ lauric acid, and the diluent Kermac 470B. The aqueous phase consisted of $30 \mathrm{mg} / \mathrm{L}$ $\mathrm{Ni}^{2+}$ and $1 M \mathrm{KNO}_{3}$. The organic to aqueous (O/A) ratio was $1: 1$ (by volume), and the total volume in the reactor was $200 \mathrm{~mL}$. The standard temperature was $25^{\circ} \mathrm{C}$, and the stirring speed was $2000 \mathrm{rpm}$.

\section{Results and Discussion}

\section{Effect of Stirring Speed}

To simplify the study, it was essential to run experiments under conditions in which the bulk diffusion rate is not limiting the reaction. Figure 1 indicates that for both $\mathrm{Lix} 65 \mathrm{~N}$ and $\mathrm{Lix} 70$ systems, when stirring speeds were higher than $2000 \mathrm{rpm}$, the extraction rate was independent of the stirring speed. Therefore, a $2000 \mathrm{rpm}$ stirring speed was used in all other experiments.

When ultrasound was used, the sonic energy caused formation of a large number of tiny droplets whose strong turbulence and propagation direction were visible. The presence of ultrasound caused a significant increase in the extraction rates, which were much faster than the rates caused by mechanical stirring. Although the effect of the mechanical stirring was negligible 


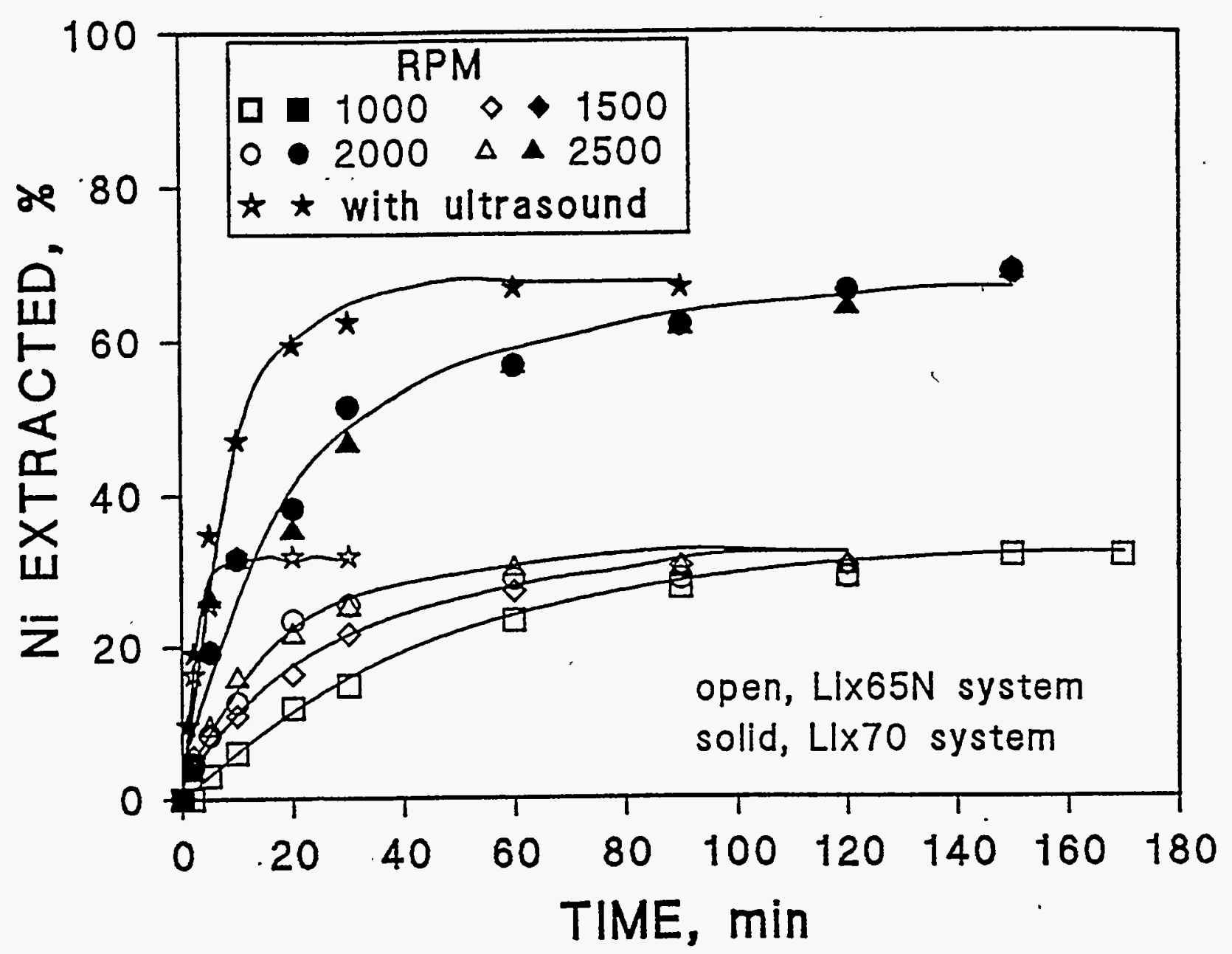

Figure 1. The effect of stirring speed on the solvent extraction of nickel with $\operatorname{Lix} 65 \mathrm{~N}$ - lauric acid - Kermac 470B and Lix 70 - lauric acid - Kermac 470B.

Organic phase: $10 \%(\mathrm{v} / \mathrm{v})$ extractant, $0.1 \mathrm{M}$ lauric acid, Kermac $470 \mathrm{~B}$.

Aqueous phase: $30 \mathrm{mg} / \mathrm{L} \mathrm{Ni}^{2+}, 1 M \mathrm{KNO}_{3}, \mathrm{pH}_{\text {init }}=5.05$.

System: $\mathrm{O} / \mathrm{A}=1: 1, T=25^{\circ} \mathrm{C}$.

Ultrasound: $20 \mathrm{kHz}, 47 \mathrm{~W}$. 
in the presence of ultrasound, the sonochemical solvent extraction study was still performed in the presence of mechanical stirring. The reason for using mechanical stirring during the sonochemical extraction was to ensure a uniform distribution of phases in the reactor (i.e., to bring the organic and aqueous phases under the ultrasonic horn).

\section{Effect of Initial pH}

Figure 2 illustrates the effect of the initial $\mathrm{pH}$ on nickel extraction with the $10 \% \mathrm{Lix} 65 \mathrm{~N}$ $0.1 M$ lauric acid - Kermac 470B system, with and without the use of ultrasound. Similar results for the Lix 70 - lauric acid - Kermac 470B system are shown in Figure 3.

Using the data presented in these two figures, the apparent forward reaction rate constant was calculated according to Flett's method, ${ }^{40}$ from the slope of the line in a plot of $\left(C_{0}-C_{e}\right) / C_{0} \ln \left[\left(C_{0}-C_{e}\right) /\left(C_{t}-C_{e}\right)\right]$ versus time, where $C$ is the nickel concentration in the aqueous phase, the subscript $t$ represents the extraction time, and the subscripts $o$ and $e$ correspond to the initial and equilibrium concentrations, respectively. The apparent forward reaction rate constants are presented in Table 1.

From Table 1, it can be seen that the use of ultrasound accelerates the extraction rates of nickel by a factor of nearly four to eight times compared to conventional extraction. The extraction rates with the Lix 70 system were slower than those with the Lix $65 \mathrm{~N}$ system, the former requiring about one hour to reach equilibrium even in the presence of ultrasound. However, the extraction yield was reversed. Comparing Figure 3 with Figure 2, it can be seen that the Lix 70 system resulted in about twice the nickel extraction yield compared to the Lix $65 \mathrm{~N}$ system. This is because of the structural difference between the active components of $\mathrm{Lix} 70$ and Lix $65 \mathrm{~N}$ extractants. The rate of the extraction with Lix 70 was slower because the steric effect of the bulky chlorine atom at the ortho position of the phenolic hydroxyl group in the molecule of $\mathrm{Lix} 70$ diminishes the complexation reaction. On the other hand, Lix 70 has a higher extraction capacity because the inductive effect of the electro-negative chlorine increases the dissociation of the hydrogen ion in the phenolic hydroxyl group.

\section{Effect of Temperature}

The effect of temperature on the solvent extraction of nickel, with and without the use of ultrasound, was studied in the range 25 to $50^{\circ} \mathrm{C}$. The results were obtained for both $\mathrm{Lix} 65 \mathrm{~N}$ and Lix 70 extractants and are presented in Figures 4 and 5 and Table 2.

Activation energies for the extraction systems were determined from Arrhenius plots of the reaction rate constants given in Table 2. These activation energies are presented in Table 3. For both extractants, Lix $65 \mathrm{~N}$ and Lix 70, and either in the presence or absence of ultrasound, the magnitude of the calculated activation energies indicates that a chemical reaction step is controlling the reaction rate.

The high activation energy for nickel extraction with the Lix reagents in the presence of ultrasonic energy presents a striking contrast to the zero activation energy obtained during the sonochemical extraction of gallium with Kelex 100 (Part III of this report). This phenomenon can be explained as a result of the different species and compositions in these two extraction systems. 


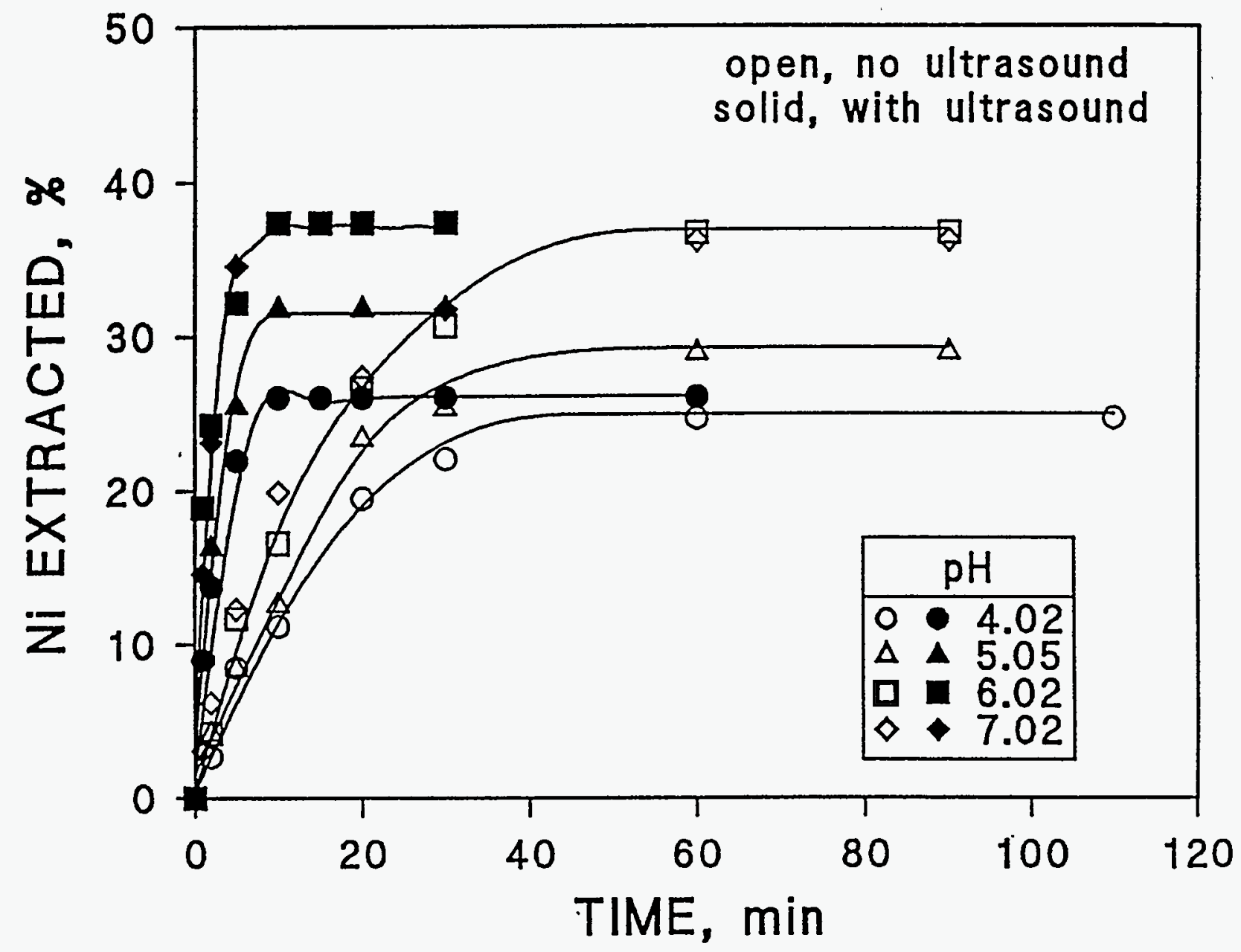

Figure 2. The effect of the initial $\mathrm{pH}$ on nickel extraction with Lix $65 \mathrm{~N}$ - lauric acid Kermac 470B.

Organic phase: $10 \%(\mathrm{v} / \mathrm{v})$ Lix $65 \mathrm{~N}, 0.1 \mathrm{M}$ lauric acid, Kermac 470B.

Aqueous phase: $30 \mathrm{mg} / \mathrm{L} \mathrm{Ni}^{2+}, 1 M \mathrm{KNO}_{3}$.

System: $\mathrm{O} / \mathrm{A}=1: 1, T=25^{\circ} \mathrm{C}$, stirring speed $2000 \mathrm{rpm}$.

Ultrasound: $20 \mathrm{kHz}, 47 \mathrm{~W}$. 


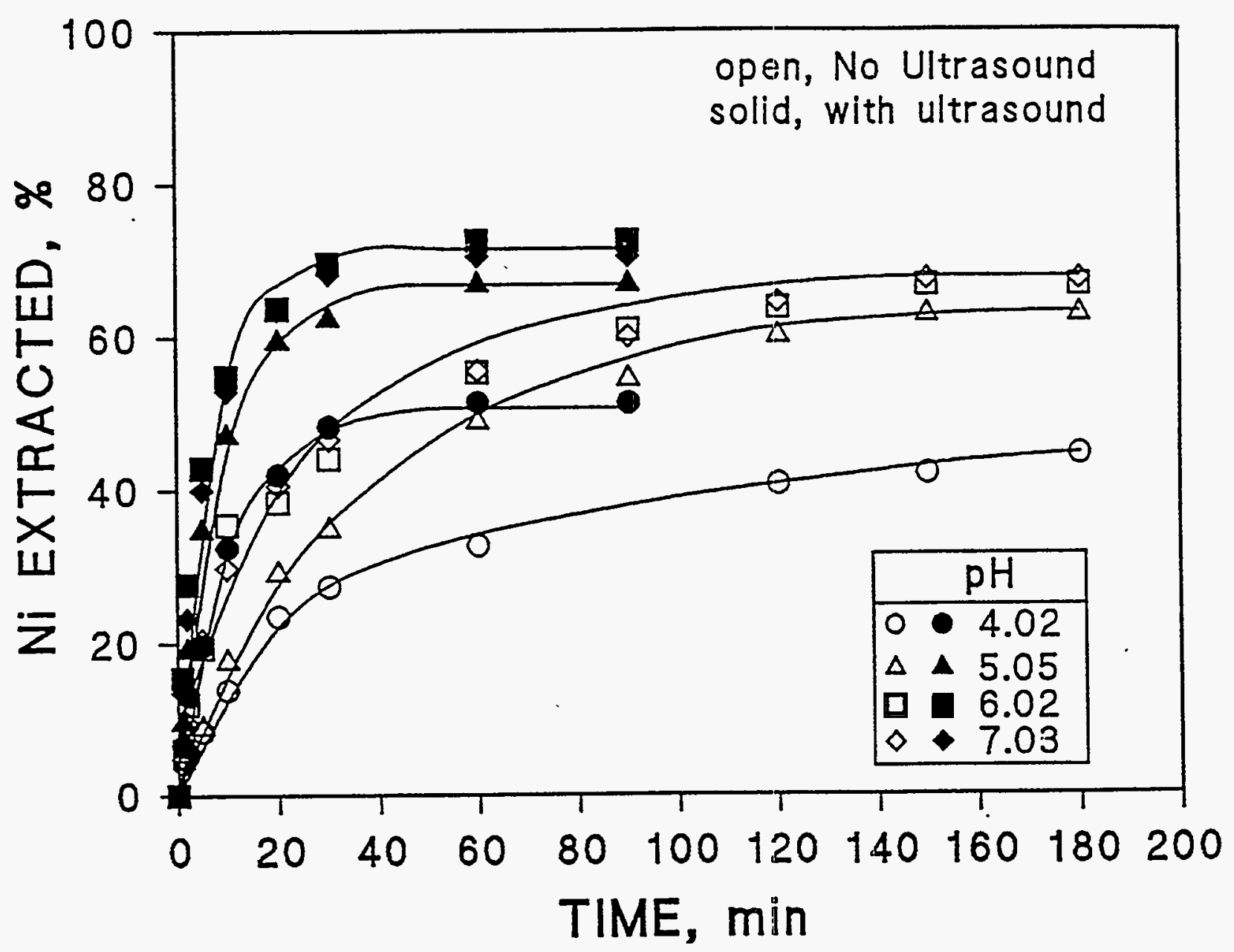

Figure 3. The effect of the initial $\mathrm{pH}$ on nickel extraction with $\mathrm{Lix} 70$ - lauric acid Kermac 470B.

Organic phase: $10 \%(\mathrm{v} / \mathrm{v}) \mathrm{Lix} 70,0.1 \mathrm{M}$ lauric acid, Kermac 470B.

Aqueous phase: $30 \mathrm{mg} / \mathrm{L} \mathrm{Ni}^{2+}, 1 M \mathrm{KNO}_{3}$.

System: $\mathrm{O} / \mathrm{A}=1: 1, T=25^{\circ} \mathrm{C}$, stirring speed $2000 \mathrm{rpm}$.

Ultrasound: $20 \mathrm{kHz}, 47 \mathrm{~W}$. 
Table 1. The effect of the initial $\mathrm{pH}$ on solvent extraction of nickel in the absence and in the presence of ultrasound.

\begin{tabular}{cccccc}
\hline & \multicolumn{4}{c}{$\begin{array}{c}\mathrm{k}_{\mathrm{f}} \times 10^{4} \\
\left(\mathrm{sec}^{-1}\right)\end{array}$} \\
\cline { 2 - 3 } & \multicolumn{2}{c}{$\begin{array}{c}\text { Lix 65N - Lauric Acid - } \\
\text { Kermac 470B }\end{array}$} & & \multicolumn{2}{c}{$\begin{array}{c}\text { Lix 70 - Lauric Acid - } \\
\text { Kermac 470B }\end{array}$} \\
\cline { 2 - 3 } \cline { 5 - 6 } $\mathrm{pH}_{\text {init }} / \mathrm{pH}_{\text {final }}{ }^{*}$ & Without & With & & Without & With \\
\hline $4.02 / 3.72$ & 3.22 & Ultrasound & & Ultrasound & Ultrasound \\
$5.05 / 3.83$ & 3.46 & 13.7 & & 1.43 & 7.83 \\
$6.02 / 3.84$ & 3.79 & 16.5 & & 2.67 & 10.0 \\
$7.02 / 3.88$ & 4.27 & 19.8 & & 2.85 & 12.0 \\
& & 33.4 & & 2.79 & 13.0
\end{tabular}

Organic phase: $10 \%(\mathrm{v} / \mathrm{v})$ hydroxyoxime - $0.1 \mathrm{M}$ lauric acid - Kermac $470 \mathrm{~B}$.

Aqueous phase: $30 \mathrm{mg} / \mathrm{L} \mathrm{Ni}{ }^{2+}, 1 M \mathrm{KNO}_{3}$.

System: $\mathrm{O} / \mathrm{A}=1: 1, T=25^{\circ} \mathrm{C}$, stirring speed $2000 \mathrm{rpm}$.

Table 2. Effect of temperature on solvent extraction of nickel in the absence and in the presence of ultrasound.

\begin{tabular}{|c|c|c|c|c|}
\hline \multirow[b]{3}{*}{$\begin{array}{c}\text { Temperature } \\
\left({ }^{\circ} \mathrm{C}\right)\end{array}$} & \multicolumn{4}{|c|}{$\begin{array}{c}\mathrm{k}_{\mathrm{f}} \times 10^{4} \\
\left(\mathrm{sec}^{-1}\right)\end{array}$} \\
\hline & \multicolumn{2}{|c|}{$\begin{array}{c}\text { Lix } 65 \mathrm{~N} \text { - Lauric Acid - } \\
\text { Kermac 470B }\end{array}$} & \multicolumn{2}{|c|}{$\begin{array}{c}\text { Lix } 70 \text { - Lauric Acid - } \\
\text { Kermac 470B }\end{array}$} \\
\hline & $\begin{array}{l}\text { Without } \\
\text { Ultrasound }\end{array}$ & $\begin{array}{c}\text { With } \\
\text { Ultrasound }\end{array}$ & $\begin{array}{l}\text { Without } \\
\text { Ultrasound }\end{array}$ & $\begin{array}{c}\text { With } \\
\text { Ultrasound }\end{array}$ \\
\hline 25 & 3.46 & 16.5 & 2.67 & 10.1 \\
\hline 40 & 5.78 & 27.1 & 7.92 & 23.6 \\
\hline 50 & 13.7 & 37.1 & 13.3 & 49.3 \\
\hline \multicolumn{5}{|c|}{$\begin{array}{l}\text { Organic phase: } 10 \%(\mathrm{v} / \mathrm{v}) \text { hydroxyoxime, } 0.1 M \text { lauric acid, Kerr } \\
\text { Aqueous phase: } \quad 30 \mathrm{mg} / \mathrm{L} \mathrm{Ni}^{2+}, 1 M \mathrm{KNO}_{3}, \mathrm{pH}_{\text {init }}=5.05 . \\
\text { System: } \mathrm{O} / \mathrm{A}=1: 1, \text { stirring speed } 2000 \mathrm{rpm} .\end{array}$} \\
\hline
\end{tabular}




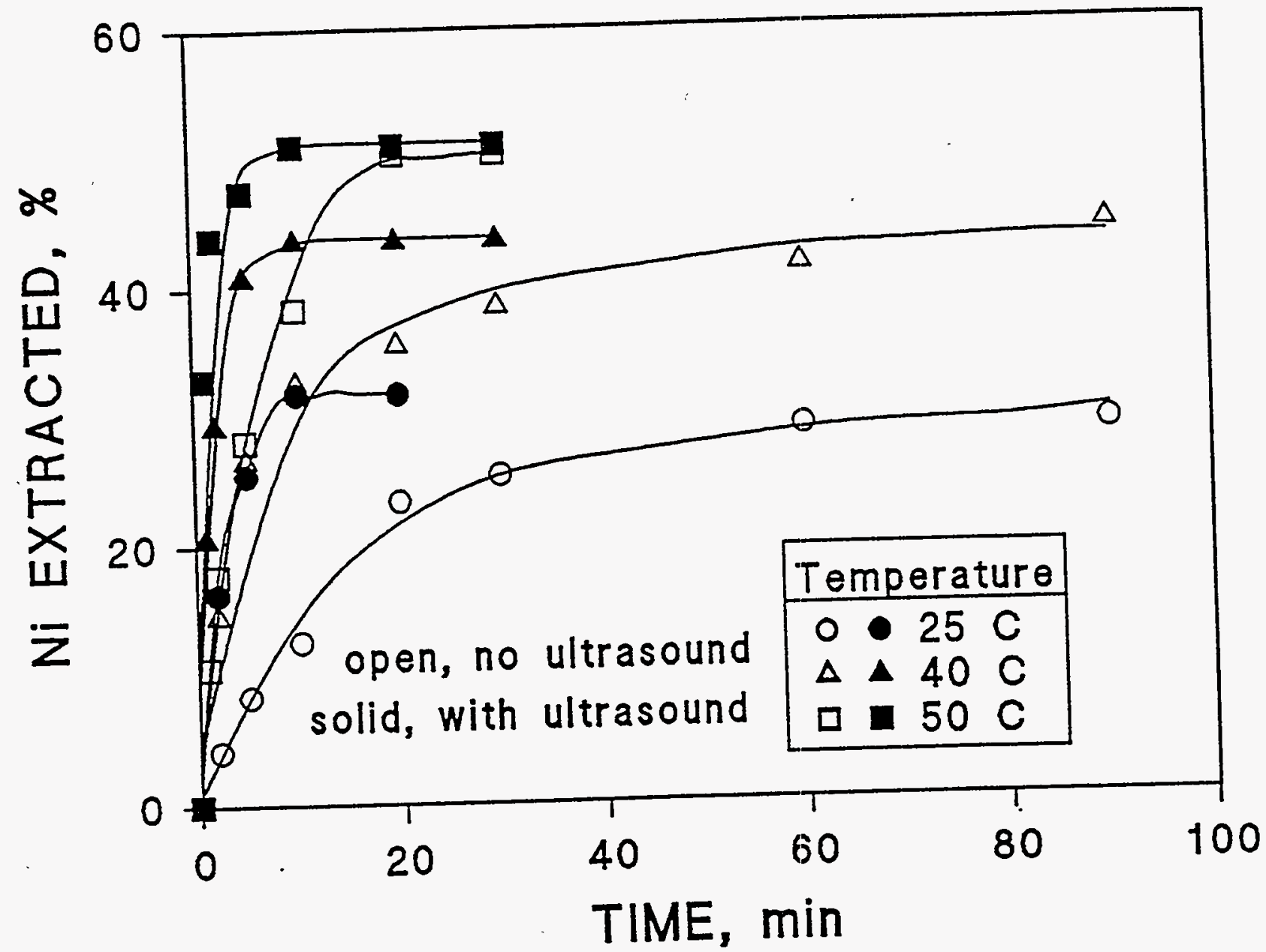

Figure 4. The effect of temperature on nickel extraction with Lix $65 \mathrm{~N}$ - lauric acid Kermac 470B.

Organic phase: $10 \%(\mathrm{v} / \mathrm{v}) \mathrm{Lix} 65 \mathrm{~N}, 0.1 \mathrm{M}$ lauric acid, Kermac 470B.

Aqueous phase: $30 \mathrm{mg} / \mathrm{L} \mathrm{Ni}^{2+}, 1 M \mathrm{KNO}_{3}, \mathrm{pH}_{\text {init }}=5.05$.

System: $\mathrm{O} / \mathrm{A}=1: 1$, stirring speed $2000 \mathrm{rpm}$.

Ultrasound: $20 \mathrm{kHz}, 47 \mathrm{~W}$. 


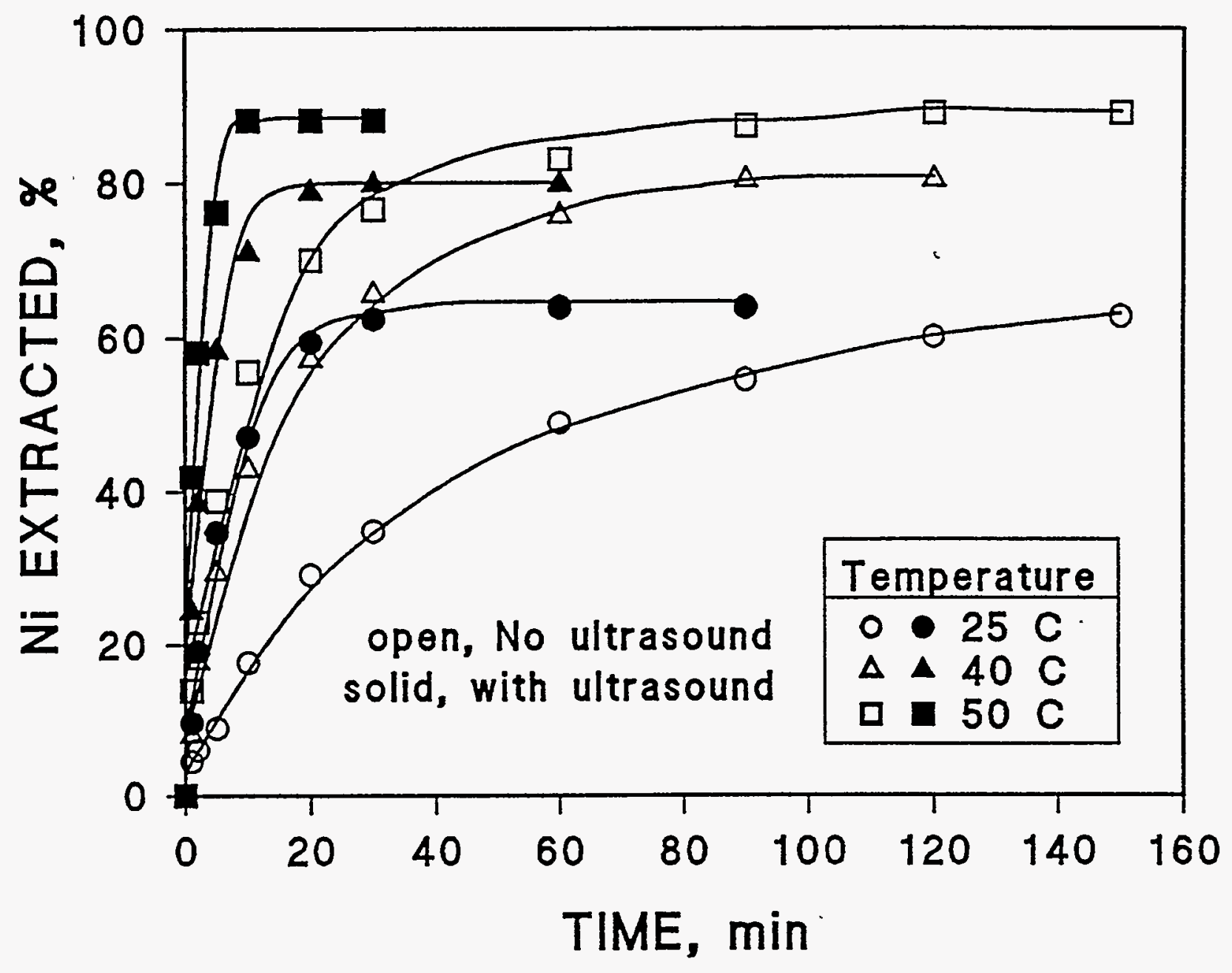

Figure 5. The effect of temperature on nickel extraction with Lix 70 - lauric acid Kermac 470B.

Organic phase: $10 \%$ (v/v) Lix 70, $0.1 M$ lauric acid, Kermac 470B.

Aqueous phase: $30 \mathrm{mg} / \mathrm{L} \mathrm{Ni}{ }^{2+}, 1 M \mathrm{KNO}_{3}, \mathrm{pH}_{\text {init }}=5.05$.

System: $\mathrm{O} / \mathrm{A}=1: 1$, stirring speed $2000 \mathrm{rpm}$.

Ultrasound: $20 \mathrm{kHz}, 47 \mathrm{~W}$. 
Table 3. Activation energy for nickel extraction in the absence and in the presence of ultrasound.

Activation Energy, $\mathrm{kJ} / \mathrm{mole}$

\begin{tabular}{ccccc}
\hline \multicolumn{2}{c}{$\begin{array}{c}\text { Lix } 65 \mathrm{~N} \text { - Lauric Acid - } \\
\text { Kermac 470B }\end{array}$} & & \multicolumn{2}{c}{$\begin{array}{c}\text { Lix 70 - Lauric Acid - } \\
\text { Kermac 470B }\end{array}$} \\
\cline { 1 - 2 } $\begin{array}{c}\text { Without } \\
\text { Ultrasound }\end{array}$ & $\begin{array}{c}\text { With } \\
\text { Ultrasound }\end{array}$ & & $\begin{array}{c}\text { Without } \\
\text { Ultrasound }\end{array}$ & $\begin{array}{c}\text { With } \\
\text { Ultrasound }\end{array}$ \\
\hline 42.4 & 35.8 & & 52.1 & 50.1 \\
\hline
\end{tabular}

Gallium belongs to a category of ions characterized by the slowest water substitution rate, whereas the rate of water substitution for nickel is several thousand times faster. Dehydration of gallate ions in the strong basic solution dominates the extraction rate of gallium (as will be shown in Part II of this report). Ultrasound can have a significant effect on dehydration and diffusion of gallate ions, which can override the thermal energy contribution to these effects. In the case of nickel extraction, the extraction rate may be controlled by the reaction of an intermediate complex between nickel and the carboxylic acid with a molecule of the hydroxyoxime. ${ }^{40}$

\section{Effect of Diluents}

Kermac 470B was used as a standard diluent in this study. Because this diluent contains about $\sim 17.3 \%$ aromatic compounds, it was decided to examine the effect of aromatic compounds on the solvent extraction of nickel. Toluene was used as a representative of the aromatic compounds. When toluene was used as a diluent during solvent extraction of nickel with Lix 70, much slower extraction rates were obtained. This can be explained by the stronger solvency of the aromatic compound. The stronger solvency of toluene may result in fewer extractant molecules ( $\mathrm{Lix} 70$ ) being present at the interface between the organic and inorganic phases.

\section{Effect of Initial Nickel Concentration}

The effect of the initial nickel concentration on its solvent extraction was examined by performing one experiment with $300 \mathrm{mg} / \mathrm{L}$ initial nickel concentration. The results of this experiment are presented in Figure 6. The experiment was performed in the presence and in the absence of ultrasound, and the results at the higher initial concentration were compared to the results obtained under the standard conditions of $30 \mathrm{mg} / \mathrm{L} \mathrm{Ni}^{2+}$. The rate of nickel extraction with Lix 70 from a solution containing $300 \mathrm{mg} / \mathrm{L}$ of nickel was -2.5 times slower, indicating that the increase in nickel concentration had a negative effect on the solvent extraction rate. Application of ultrasound in the system with $300 \mathrm{mg} / \mathrm{L} \mathrm{Ni}{ }^{2+}$ increased the rate of extraction by a factor of $\sim 3$. Ultrasound did not enhance the capacity of the extractant in the system studied. 


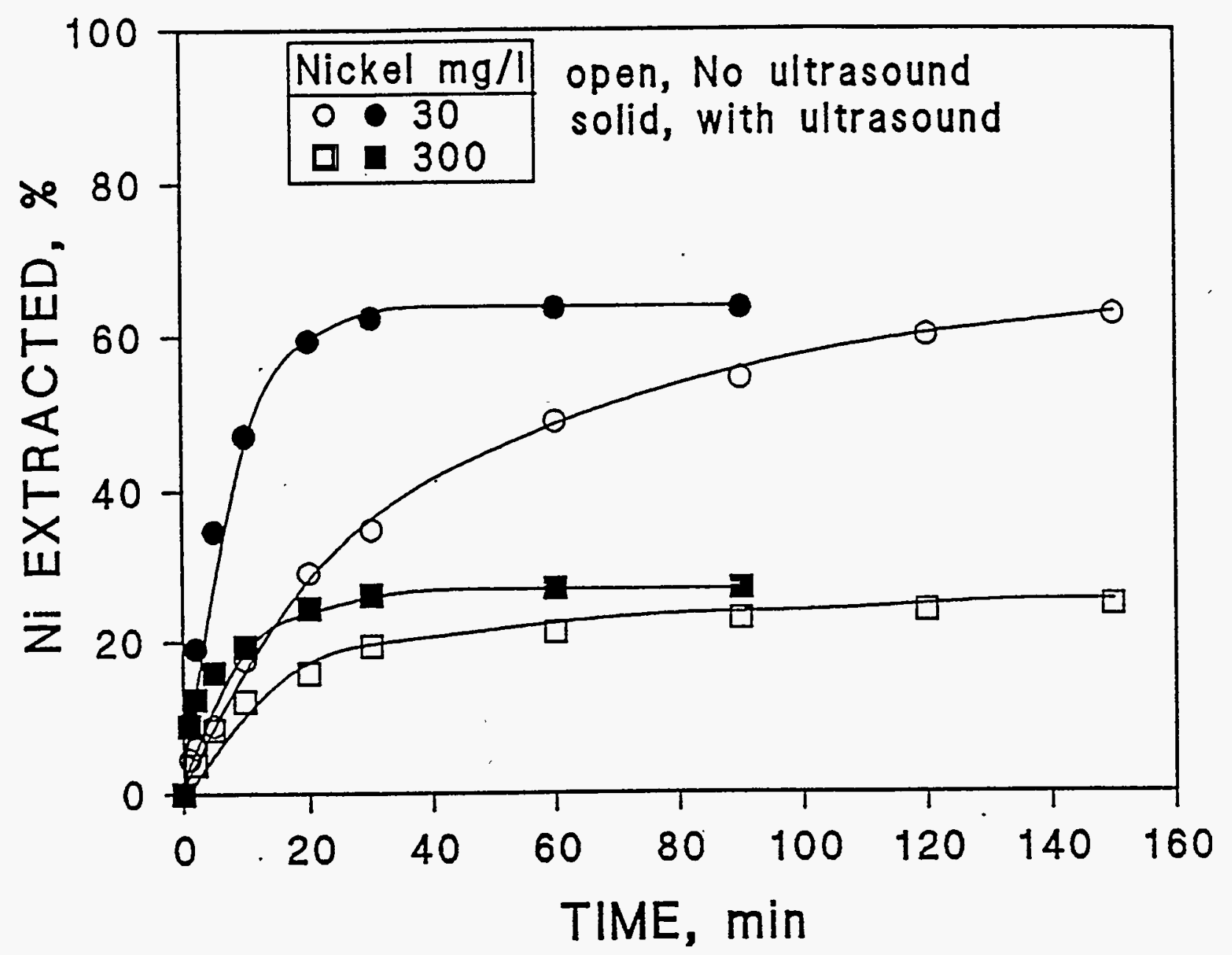

Figure 6. The effect of initial nickel concentration on nickel extraction with $\mathrm{Lix} 70$ - lauric acid Kermac 470B.

Organic phase: $10 \%$ (v/v) Lix 70, $0.1 M$ lauric acid, Kermac 470B.

Aqueous phase: $\mathrm{Ni}^{2+}, 1 M \mathrm{KNO}_{3}, \mathrm{pH}_{\text {init }}=5.05$.

System: $\mathrm{O} / \mathrm{A}=1: 1, T=25^{\circ} \mathrm{C}$, stirring speed $2000 \mathrm{rpm}$.

Ultrasound: $20 \mathrm{kHz}, 47 \mathrm{~W}$. 


\section{Effect of Ultrasound on Equilibrium Extraction Loading}

The equilibrium extraction loading isotherms for the solvent extraction of nickel with Lix $65 \mathrm{~N}$ and $\mathrm{Lix} 70$, with and without the use of ultrasound, are presented in Figures 7 and 8. From examination of the figures it can be concluded that ultrasound does not have any effect on the equilibrium extraction loading.

\section{Extraction of Cobalt and Separation of Cobalt and Nickel}

The effect of ultrasound on the solvent extraction of cobalt was studied with the Lix 70 lauric acid - Kermac 470B system and the results are presented in Figure 9. As in the case with nickel, the extraction of cobalt with $\mathrm{Lix} 70$ was slow, requiring $-3 \mathrm{~h}$ to achieve equilibrium. In the presence of ultrasound, the cobalt extraction rate increased substantially, but a more important benefit was the increased extraction yield. The uptake of cobalt increased from $\sim 30 \%$ without ultrasound to $\sim 75 \%$ in the presence of ultrasound. However, it was found that cobalt stripping from the loaded organic phase was difficult, especially when the loading was performed at higher initial $\mathrm{pH}$. Cobalt stripping from the loaded organic phase was possible only in the presence of a reductant, such as sodium sulfite.

In normal extraction systems (without ultrasound), it had been found that the solvent extraction of cobalt with a substituted hydroxyoxime alone can lead to oxidation of cobalt (by oxygen from air) to a +3 valence state, which, when extracted, becomes difficult to strip. ${ }^{45}$ The role of lauric acid in the system studied here was to slow the cobalt oxidation at the interface as a result of the interfacial activity of the carboxylic acid. However, when ultrasound was applied, the interfacial surface area increased significantly, causing conditions for enhanced cobalt oxidation to the +3 valence state.

The results of a test of solvent extraction from solutions containing a mixture of nickel and cobalt in the absence of ultrasound are presented in Figure 10. These results show that the presence of nickel suppressed the extraction of cobalt. While cobalt extraction equilibrium was established in $10 \mathrm{~min}$, the extraction of nickel continued throughout the experiment. The net effect of this phenomenon was that the separation of nickel from cobalt increased.

In the presence of ultrasound the situation was reversed, as shown in Figure 11. Cobalt extraction continued throughout the experiment, while nickel extraction reached equilibrium in 30 min. Thus the presence of ultrasound had a negative effect on separation of nickel and cobalt. This result can also be used as additional evidence that cobalt oxidation was occurring in the presence of ultrasound, which permitted the continued extraction of cobalt to take place.

\section{Effect of Surfactants}

A surfactant plays an important role in the kinetics of solvent extraction of metals. The addition of the surfactant DNNSA even at a low concentrations increased the extraction rate of nickel in the hydroxyoxime - lauric acid - Kermac 470B systems. The rate increase can be assigned to the fact that DNNSA promotes an increase in the interfacial surface area between the organic and inorganic liquid phases during mixing. Also, this active sulfonic acid molecule is preferentially oriented at the interface, and can itself extract nickel. ${ }^{46}$ 


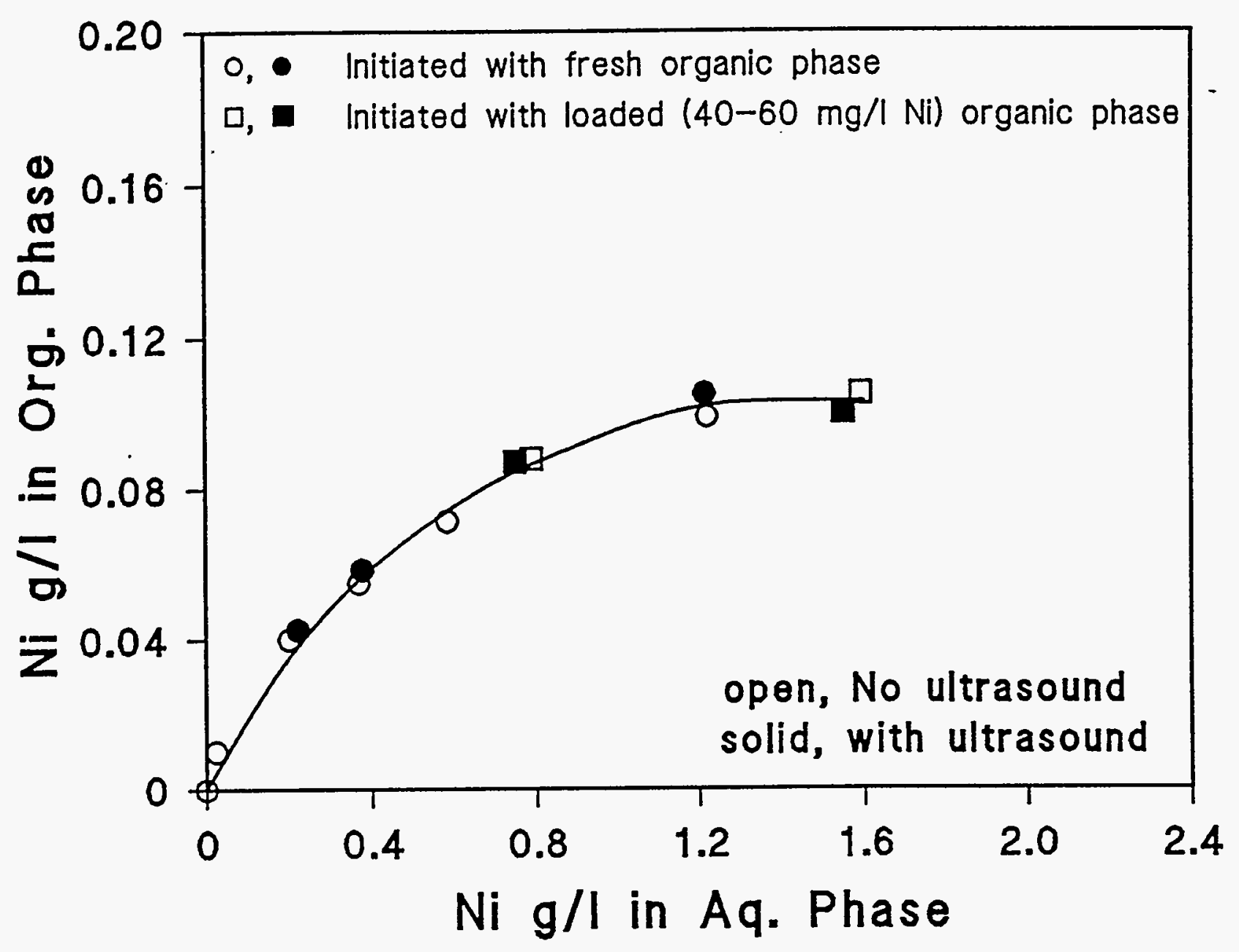

Figure 7. Extraction isotherm of nickel with Lix $65 \mathrm{~N}$ - lauric acid - Kermac 470B.

Organic phase: $10 \%(v / v)$ Lix $65 \mathrm{~N}, 0.1 \mathrm{M}$ lauric acid, Kermac $470 \mathrm{~B}$.

Aqueous phase: $\mathrm{Ni}^{2+}, 1 M \mathrm{KNO}_{3}, \mathrm{pH}_{\text {eq }}=3.2$ to 3.3.

System: $T=25^{\circ} \mathrm{C}$, stirring speed $2000 \mathrm{rpm}$.

Ultrasound: $20 \mathrm{kHz}, 32.5 \mathrm{~W}$. 


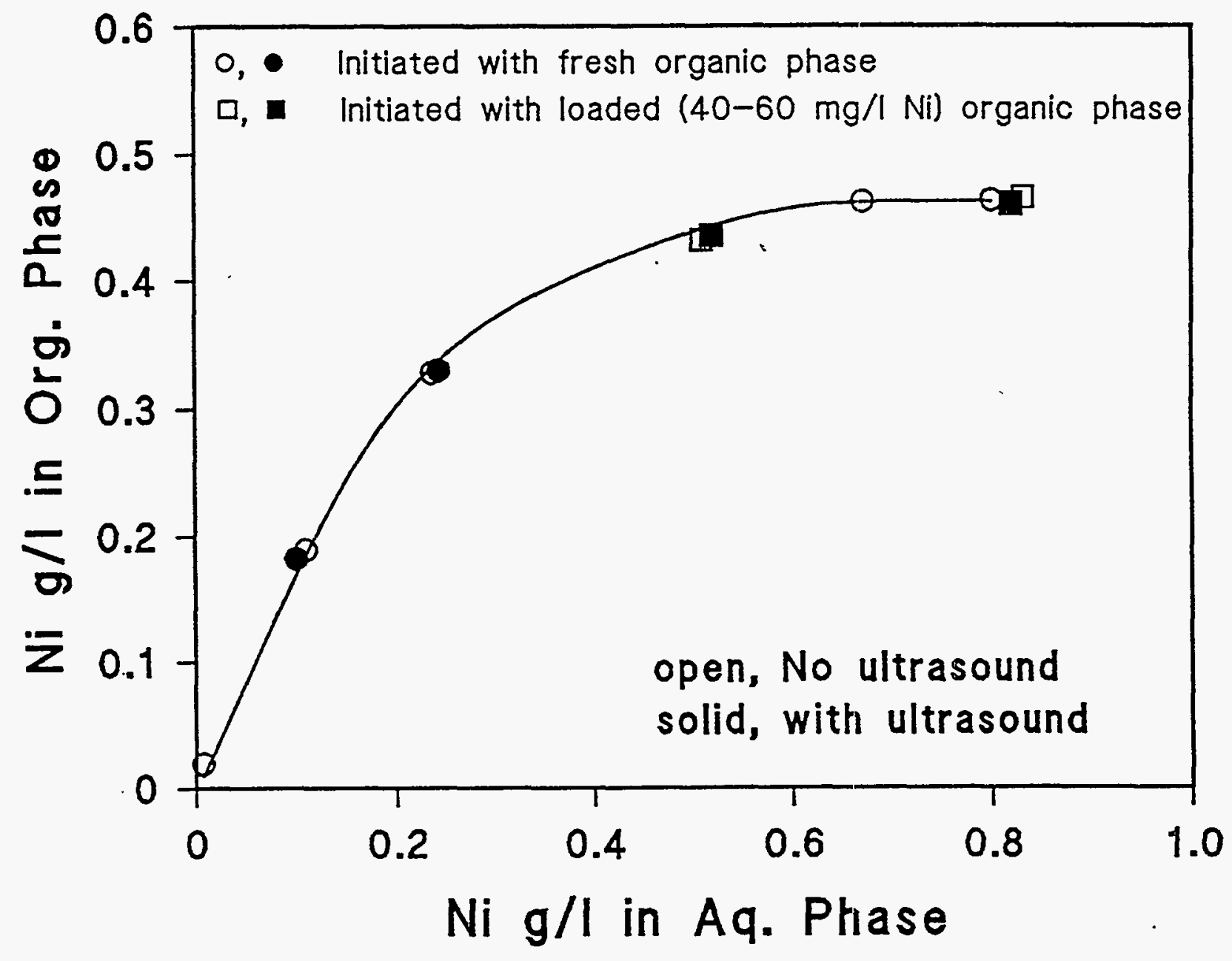

Figure 8. Extraction isotherm of nickel with Lix 70 - lauric acid - Kermac 470B. Organic phase: $10 \%$ (v/v) Lix 70, $0.1 M$ lauric acid, Kermac 470B. Aqueous phase: $\mathrm{Ni}^{2+}, 1 M \mathrm{KNO}_{3}, \mathrm{pH}_{\mathrm{eq}}=2.9$ to 3.1 . System: $T=25^{\circ} \mathrm{C}$, stirring speed $2000 \mathrm{rpm}$. Ultrasound: $20 \mathrm{kHz}, 32.5 \mathrm{~W}$. 


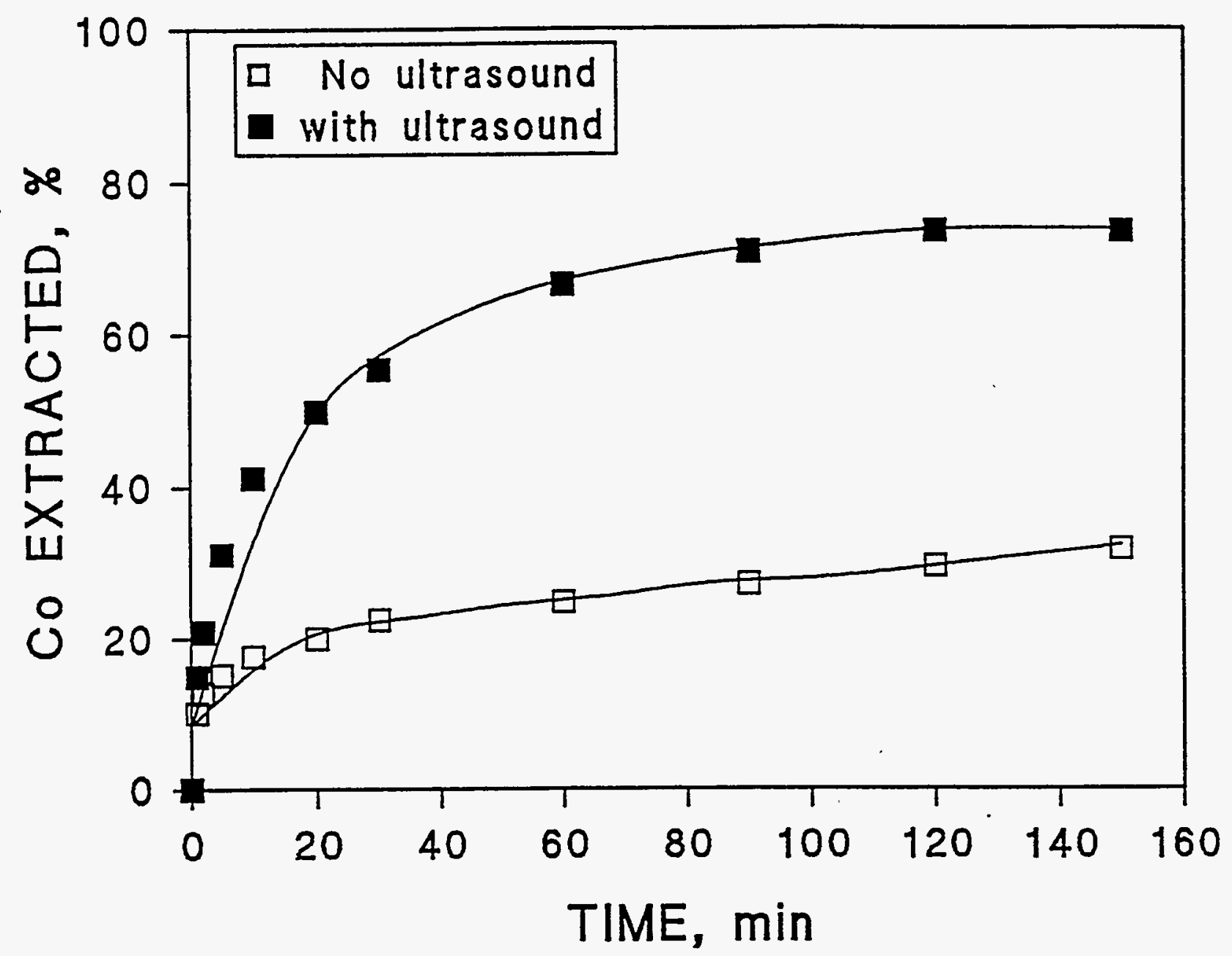

Figure 9. The effect of ultrasound on the solvent extraction of Cobalt with Lix 70 - lauric acid Kermac 470B at $\mathrm{pH} 5$.

Organic phase: $10 \%(\mathrm{v} / \mathrm{v}) \mathrm{Lix} 70,0.1 \mathrm{M}$ lauric acid, Kermac 470B.

Aqueous phase: $30 \mathrm{mg} / \mathrm{L} \mathrm{Co}^{2+}, 1 M \mathrm{KNO}_{3}, \mathrm{pH}_{\text {init }}=5.05$.

System: $\mathrm{O} / \mathrm{A}=1: 1, T=25^{\circ} \mathrm{C}$, stirring speed $2000 \mathrm{rpm}$.

Ultrasound: $20 \mathrm{kHz}, 47 \mathrm{~W}$. 


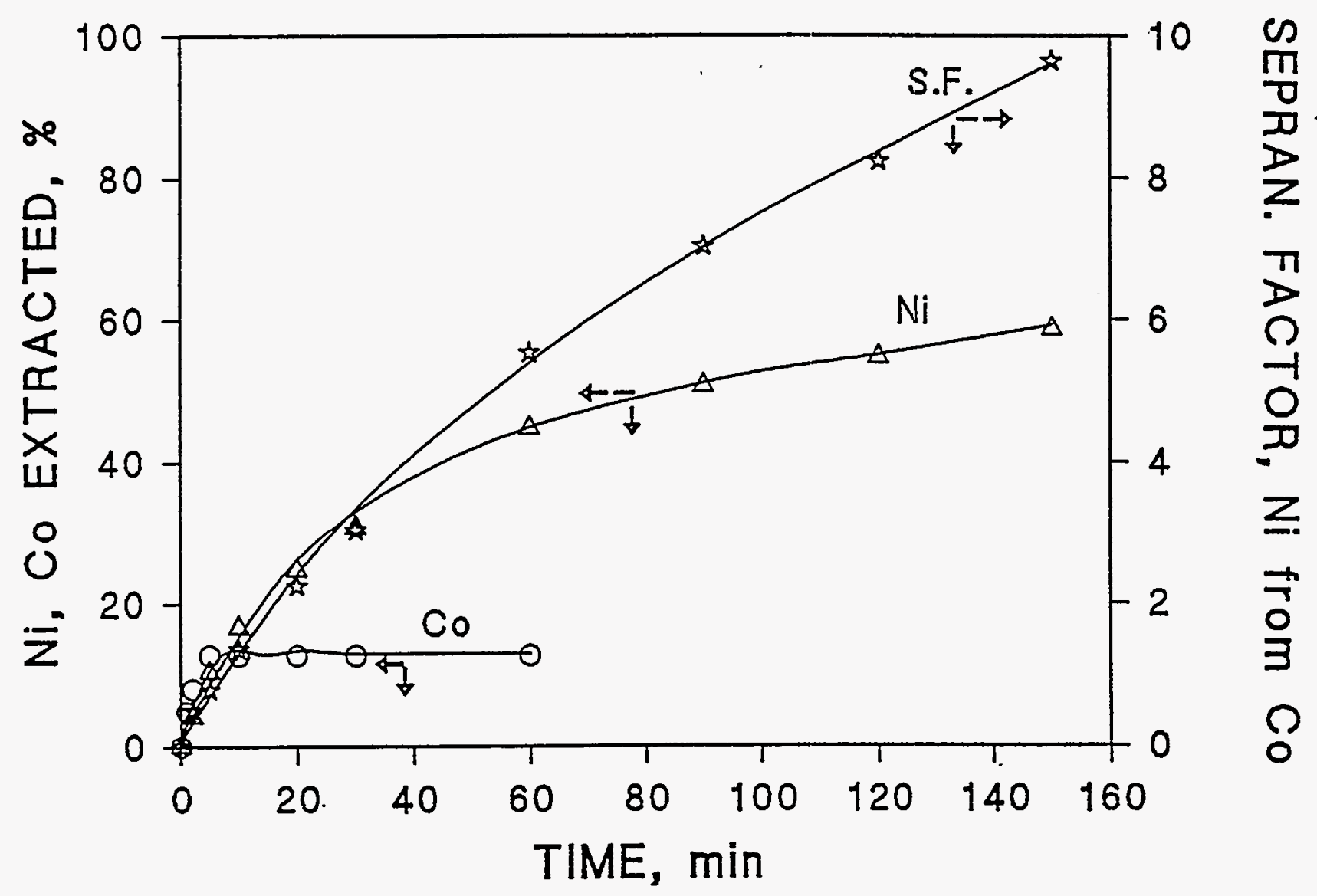

Figure 10. The extraction of nickel and Co with Lix 70 - lauric acid - Kermac 470B in the absence of ultrasound.

Organic phase: $10 \%(\mathrm{v} / \mathrm{v})$ Lix $70,0.1 M$ lauric acid, Kermac $470 \mathrm{~B}$.

Aqueous phase: $30 \mathrm{mg} / \mathrm{L} \mathrm{Co}{ }^{2+}$ and $30 \mathrm{mg} / \mathrm{L} \mathrm{Ni}^{2+}, 1 M \mathrm{KNO}_{3}, \mathrm{pH}_{\text {init }}=5.05$.

System: $\mathrm{O} / \mathrm{A}=1: 1, T=25^{\circ} \mathrm{C}$, stirring speed $2000 \mathrm{rpm}$. 


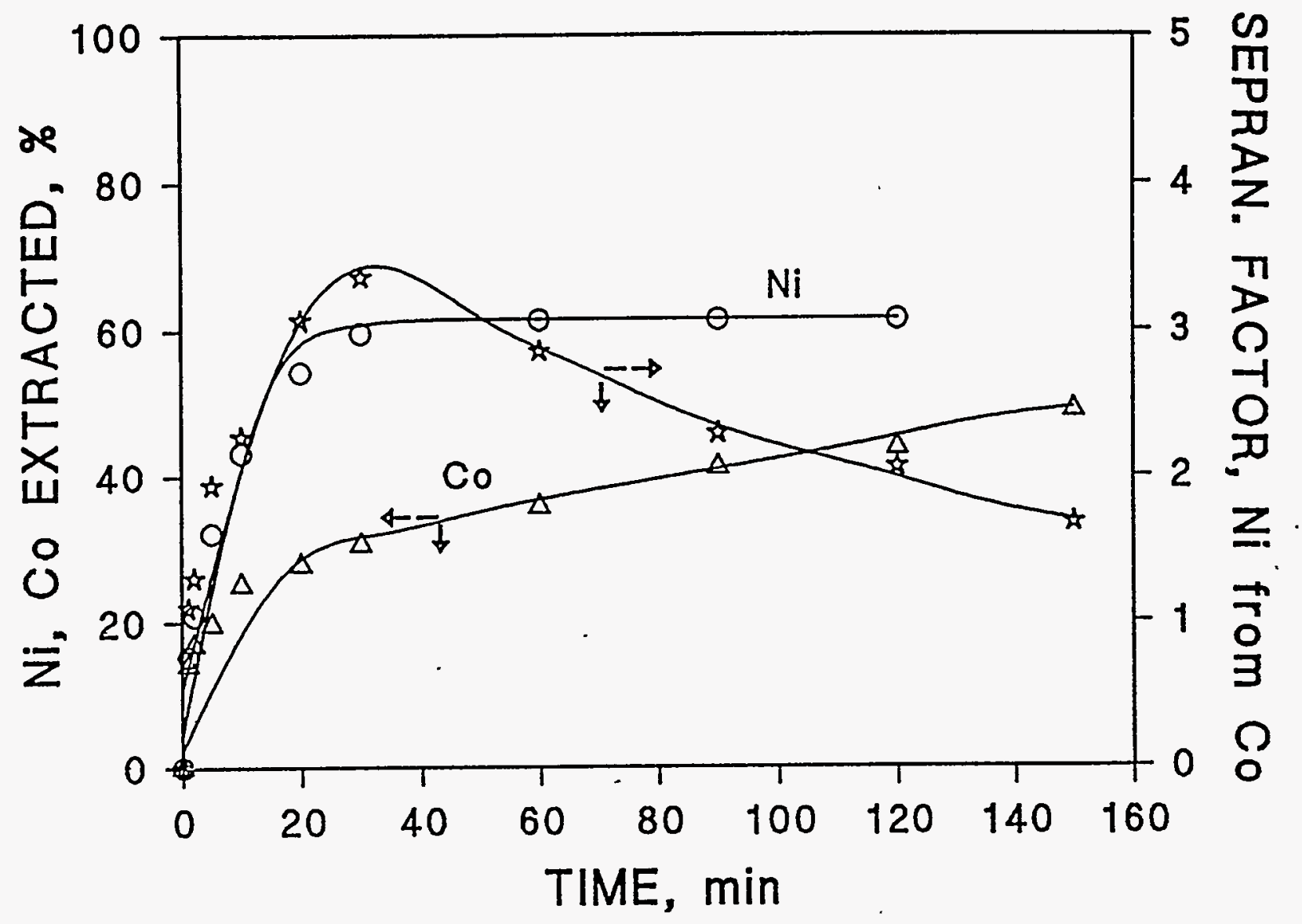

Figure 11. The extraction of nickel and Co with Lix 70 - lauric acid - Kermac 470B in the presence of ultrasound.

Organic phase: $10 \%(\mathrm{v} / \mathrm{v}) \mathrm{Lix} 70,0.1 \mathrm{M}$ lauric acid, Kermac 470B.

Aqueous phase: $30 \mathrm{mg} / \mathrm{L} \mathrm{Co}^{2+}$ and $30 \mathrm{mg} / \mathrm{L} \mathrm{Ni}^{2+}, 1 M \mathrm{KNO}_{3}, \mathrm{pH}_{\text {init }}=5.05$.

System: $\mathrm{O} / \mathrm{A}=1: 1, T=25^{\circ} \mathrm{C}$, stirring speed $2000 \mathrm{rpm}$.

Ultrasound: $20 \mathrm{kHz}, 47 \mathrm{~W}$. 
The times required to achieve equilibrium when the surfactant DNNSA or ultrasound are used are provided in Table 4. Both DNNSA and ultrasound increase the rate of extraction (decrease the time to equilibrium) by promoting the development of increased interfacial surface area. From examination of Table 4, it can be seen that the use of ultrasound had a larger effect than the addition of DNNSA.

For oil and water systems to form an emulsion, an appropriate surfactant and co-surfactant is required. Bauer and Komornicki ${ }^{47}$ used sodium laurylsulfate and n-pentanol instead of DNNSA to produce an appropriate microemulsion in the Lix 70 - Versatic 911 - kerosene system. Very fast nickel extraction kinetics were obtained. The effects of this surfactant were not compared with the effects of ultrasound in this study.

\section{Stability of the Extractant}

It is important to determine if the powerful cavitation effects produced by ultrasound can degrade the extractant molecules. Two tests were performed to examine the stability of organic molecules in the presence of ultrasound.

In the first test, a dried and undiluted sample of Lix $65 \mathrm{~N}$ was continuously subjected to ultrasound using a power input of $47 \mathrm{~W}$ for $6 \mathrm{~h}$. The solution temperature was $60^{\circ} \mathrm{C}$. Samples of Lix $65 \mathrm{~N}$ were taken before and after the test and were inspected with IR and NMR spectroscopy. The IR and NMR spectra were found to be the same, indicating that Lix $65 \mathrm{~N}$ did not change.

The second test involved multiple cycles of loading and stripping of the organic phase. If ultrasound had a negative effect on the extractant molecule, then the uptake of nickel would decrease with increasing number of cycles. Five multiple cycle tests were performed with the Lix $65 \mathrm{~N}$ - lauric acid - Kermac 470B system. The results of this test, presented in Table 5, indicate that the Lix $65 \mathrm{~N}$ molecule did not change after multiple cycles.

Table 4. Comparison of the effect of DNNSA as a surfactant and ultrasound on the time to reach equilibrium for nickel extraction.

Time to Equilibrium

(min)

\begin{tabular}{cccc}
$\begin{array}{c}\text { Ultrasound } \\
(47 \mathrm{~W})\end{array}$ & $\begin{array}{c}\text { Surfactant } \\
(\mathrm{mol} / \mathrm{L})\end{array}$ & Lix 65N System & Lix 70 System \\
\hline No & No & 60 & 150 \\
Yes & No & 10 & 60 \\
No & $0.005 M$ DNNSA & 30 & 140 \\
Yes & $0.005 M$ DNNSA & -- & 60 \\
\hline
\end{tabular}

Organic phase: $10 \%(\mathrm{v} / \mathrm{v})$ hydroxyoxime, $0.1 \mathrm{M}$ lauric acid, Kermac $470 \mathrm{~B}$.

Aqueous phase: $30 \mathrm{mg} / \mathrm{L} \mathrm{Ni}^{2+}, 1 M \mathrm{KNO}_{3}, \mathrm{pH}_{\text {init }}=5.05$.

System: $\mathrm{O} / \mathrm{A}=1: 1, T=25^{\circ} \mathrm{C}$, stirring speed $2000 \mathrm{rpm}$. 
Table 5. Multiple cycle test for extraction of nickel with Lix $65 \mathrm{~N}$ - lauric acid - Kermac 470B.

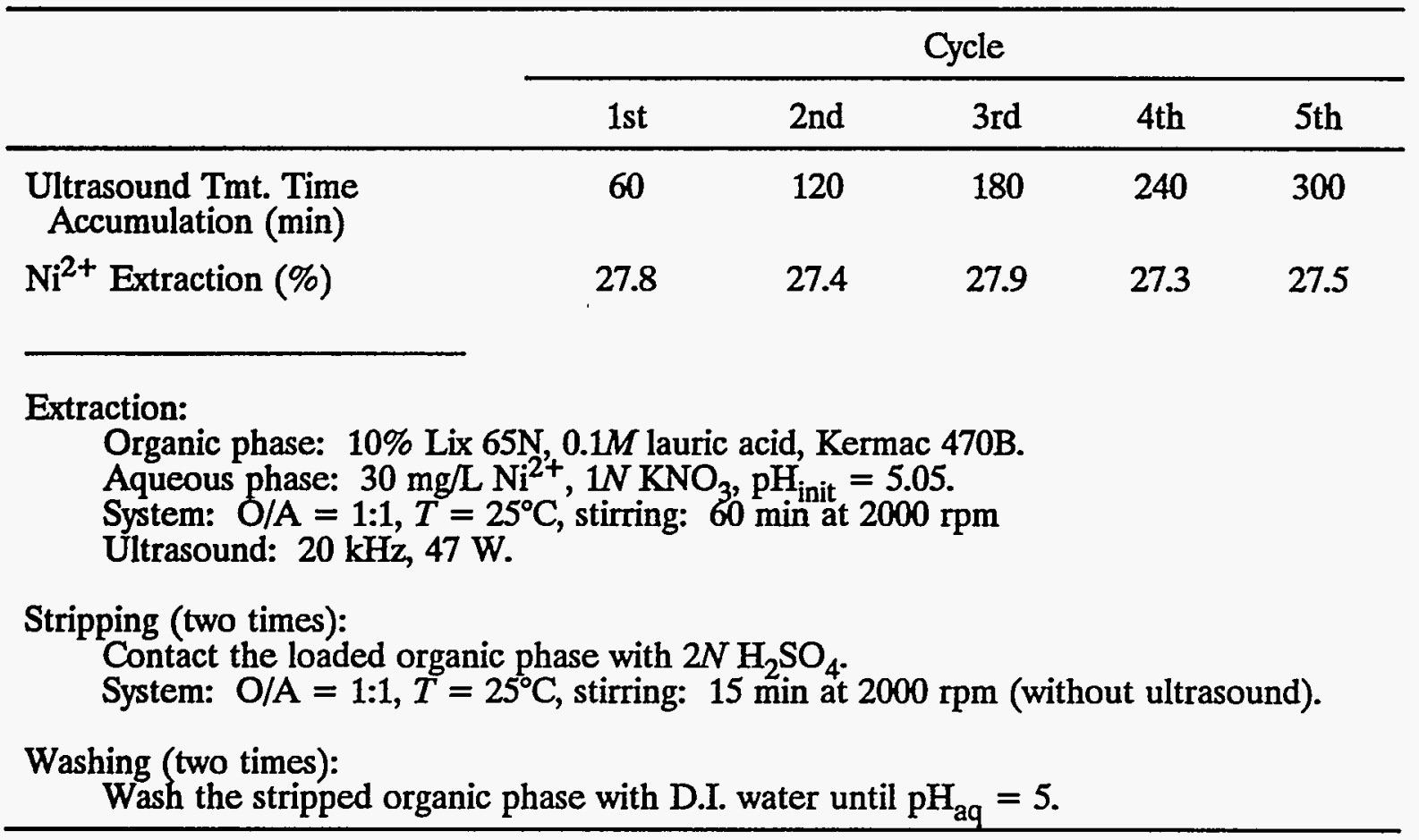

\section{Effect of Ultrasonic Frequency}

To study the effect of higher frequency ultrasound on solvent extraction, a separate reactor . was designed. A $2 \mathrm{MHz}$ ultrasonic transducer and associated electronic components were obtained by dismantling an ultrasonic humidifier. The transducer was mounted on the bottom of the reactor. The $200 \mathrm{~mL}$ reactor was made of an acrylic plastic. An experiment was performed by adding $100 \mathrm{~mL}$ of organic and $100 \mathrm{~mL}$ of aqueous phase into the reactor, after which the ultrasonic power was turned on.

It was found that the high frequency ultrasound did not have enough power to cause mixing of organic and aqueous phases. For that reason, the extraction of nickel with both extractants, Lix $65 \mathrm{~N}$ and Lix 70, was very poor. The absence of cavitation was responsible for the poor performance of the high frequency ultrasound. It is known that cavitation effects can be produced only at low frequencies, the most intense ones in the range of $20-40 \mathrm{kHz}$.

\section{Energy Consumption}

In all of the previous experiments, ultrasound was applied continuously. In such batch applications, it was found that the total energy consumed was prohibitively high, and could not be offset by the substantially shorter extraction time. However, an important discovery was made while performing an experiment on the solvent extraction of nickel with Lix $65 \mathrm{~N}$ in the presence of ultrasound. It was found that the ultrasound did not have to be continuously present in order to extract the nickel. When the ultrasound was applied for a short time and then discontinued, solvent extraction still occurred because the microdroplets that formed during the presence of 
ultrasound did not disappear immediately after ultrasound was discontinued. When a significant number of droplets coalesced, the ultrasound was resumed to form a new set of droplets in order to continue extraction.

Therefore, it was decided to examine the effect of an intermittent application of ultrasound on the solvent extraction of nickel. The intermittent application was characterized by the time when the ultrasound was "on" and the time when the ultrasound was "off." The total (on + off) time was fixed at $5 \mathrm{sec}$; thus, the frequency of the (on + off) cycles was fixed at $12 / \mathrm{min}$. The ultrasound generator was turned on and off with an electronic controller. The energy consumption during the extraction was monitored by a $W \cdot h$ meter. In addition to the on and offtimes, the intensity (power) of the ultrasound was also varied. The results of this experiment are presented in Table 6. From examination of these results, it can be noted that the lowest total energy required to achieve equilibrium occurred when the on-time was $0.25 \mathrm{sec}$ and the off-time was $4.75 \mathrm{sec}$, with an ultrasonic input power of $61 \mathrm{~W}$. Under these conditions, the total ultrasonic energy consumed in reaching equilibrium was $2.66 \mathrm{~W} \cdot \mathrm{h}$ for the $200 \mathrm{~mL}$ batch system. It is important to note that the on-time can be substantially decreased by increasing the ultrasonic power applied. Even though higher power was used, the overall energy consumption is still lower because of the shorter on-time per cycle.

When a similar study was performed with the Lix 70 , it was found that the intermittent application of ultrasound was not as effective as with Lix $65 \mathrm{~N}$. The reason for this result is due to the stability of the droplets. With the Lix 70 system, the droplet stability was much lower than with the Lix $65 \mathrm{~N}$ system, resulting in a faster coalescence and reduced interfacial surface area. To achieve the same level of extraction, longer on-times were required with $\mathrm{Lix} 70$.

Table 6. Ultrasonic energy consumption during nickel extraction with $\mathrm{Lix} 65 \mathrm{~N}$; ultrasound was used in intermittent mode.

\begin{tabular}{cccccc}
\hline \multicolumn{2}{c}{ Ultrasound Energy } & & \multicolumn{2}{c}{ Ultrasound Action } & \\
\cline { 1 - 2 } $\begin{array}{c}\text { Power } \\
\text { Input }\end{array}$ & $\begin{array}{c}\text { Energy } \\
\text { Consumption } \\
(\mathrm{W})\end{array}$ & & $\begin{array}{c}\text { Time On }) \\
(\mathrm{sec})\end{array}$ & $\begin{array}{c}\text { Time Off } \\
(\mathrm{sec})\end{array}$ & $\begin{array}{c}\text { Total Treatment Time to } \\
\text { Achieve Equilibrium Loading } \\
(\mathrm{min})\end{array}$ \\
\hline 21 & 9.56 & & 0.25 & 4.75 & 90 \\
21 & 9.41 & & 0.50 & 4.50 & 60 \\
21 & 7.76 & & 1.00 & 4.00 & 30 \\
21 & 6.71 & & 2.00 & 3.00 & 15 \\
41 & 3.81 & & 0.25 & 4.75 & 30 \\
61 & 2.66 & & 0.25 & 4.75 & 15 \\
\hline
\end{tabular}

Organic phase: $10 \%(\mathrm{v} / \mathrm{v}) \mathrm{Lix} 65 \mathrm{~N}, 0.1 \mathrm{M}$ lauric acid, Kermac $470 \mathrm{~B}$.

Aqueous phase: $30 \mathrm{mg} / \mathrm{L} \mathrm{Ni}^{2+}, 1 M \mathrm{KNO}_{3}, \mathrm{pH}_{\text {init }}=5.05$.

System: $\mathrm{O} / \mathrm{A}=1: 1$, stirring speed $600 \mathrm{rpm}$.

Ultrasonic pulse: 12 cycles/min. 
The encouraging results obtained during the intermittent application of ultrasound in the Lix $65 \mathrm{~N}$ system suggested that the energy reduction can also be achieved by continuous flow solvent extraction. For this study, a separate reactor was designed. It consisted of two holding containers (one for the organic phase and one for the aqueous phase), an ultrasonic chamber, and a receiving container. The role of the ultrasonic chamber was to receive separate organic and aqueous phases and to mix them using ultrasound. After the ultrasonic treatment, the mixed phases were pumped into a receiving container. The ultrasonic chamber was water-jacketed to control the extraction temperature. The residence time in the ultrasonic chamber was varied by changing the pumping rate of the organic and aqueous phases. The total energy consumption was monitored by a $\mathrm{W} \cdot \mathrm{h}$ meter. The results of this experiment are presented in Table 7 . The energy consumption is expressed in $\mathrm{kW} / \mathrm{h} / \mathrm{m}^{3}$ of solution. From examination of the table, it can be seen that the energy consumption can be reduced by decreasing the residence time in the ultrasonic chamber or by increasing the temperature of the solvent extraction process.

For the conditions studied here, the lowest energy consumption was achieved at the highest temperature, $26^{\circ} \mathrm{C}$, and at the highest flow rate, $160 \mathrm{~mL} / \mathrm{min}$. Higher flow rates were not examined because of the limitations of the experimental setup. Although the given experimental setup had limitations with respect to the maximum flow rate used and the accuracy of the calculated energy consumption, it still provided a clear concept of how to reduce energy consumption.

Unfortunately, the lowest energy consumption obtained was $11 \mathrm{~kW} \cdot \mathrm{h} / \mathrm{m}^{3}$. This is still too high to compete with the conventionally mixed solvent extraction process, where the upper energy consumption limit is $\sim 1 \mathrm{~kW} \cdot \mathrm{h} / \mathrm{m}^{3}$. However, the results from the continuous flow experiment and the experiment with the intermittent application of ultrasound clearly suggest that optimization studies to further reduce energy consumption need to be conducted. Perhaps a

Table 7. Ultrasonic energy consumption during solvent extraction of nickel by using a continuous flow of organic and aqueous phases.

\begin{tabular}{ccccc}
\hline $\begin{array}{c}\text { Flow Rate } \\
\text { Organic } \\
(\mathrm{mL} / \mathrm{min})\end{array}$ & $\begin{array}{c}\text { Flow Rate } \\
\text { Aqueous } \\
(\mathrm{mL} / \mathrm{min})\end{array}$ & $\begin{array}{c}\text { Temperature } \\
\left({ }^{\circ} \mathrm{C}\right)\end{array}$ & $\begin{array}{c}\text { Residence Time } \\
(\mathrm{min})\end{array}$ & $\begin{array}{c}\text { Specific Energy } \\
\text { Consumption } \\
\left(\mathrm{kW} \cdot \mathrm{h} / \mathrm{m}^{3}\right)\end{array}$ \\
\hline 10 & 10 & 15 & 10 & 176 \\
20 & 20 & 15 & 5 & 88 \\
40 & 40 & 15 & 2.5 & 88 \\
40 & 40 & 26 & 2.5 & 44 \\
80 & 80 & 26 & 1.25 & 22 \\
160 & 160 & 26 & 0.625 & 11 \\
\hline
\end{tabular}

Experimental conditions:

Organic phase: $10 \%(v / v)$ Lix $65 \mathrm{~N}, 0.1 \mathrm{M}$ lauric acid, Kermac $470 \mathrm{~B}$.

Aqueous phase: $30 \mathrm{mg} / \mathrm{L} \mathrm{Ni}^{2+}, 0.5 M \mathrm{KNO}_{3}, \mathrm{pH}_{\text {init }}=5.05$.

Ultrasound: $20 \mathrm{kHz}, 47 \mathrm{~W}$ power input. 
useful solvent extraction device would be one that utilizes a pipe as a mixer for mixing the organic and aqueous phases with properly spaced ultrasonic horns inserted through the pipe wall. According to this concept, the pipe length between the leaching reactor and the settler would serve as a mixer, thus avoiding the need for a mixer in the traditional mixer/settler design.

\section{Conclusions for Part I}

Based on the experiments described in Part I of this report, the following conclusions have been drawn:

- Ultrasound has a significantly positive effect on the solvent extraction of nickel with Lix $65 \mathrm{~N}$ and Lix 70 extractants. The extraction rate was found to increase by a factor of about four to eight.

- The presence of ultrasound results in an increased interfacial surface area, thus increasing the overall rate of extraction.

- Ultrasound does not affect the extraction equilibrium.

- Ultrasound does not need to be continuously present in order to modify the rate of the solvent extraction of nickel. It was found that an intermittent application of ultrasound could produce the same results as a continuous application. This has practical significance because it represents a method of reducing the overall energy consumption when using ultrasound.

- Because intermittent application of ultrasound produced results similar to continuous application, and because ultrasound did not have any effect on equilibrium of extraction, it can be concluded that ultrasound does not have any effect on the chemical reaction mechanisms. The effect of ultrasound is of a physical nature, increasing the interfacial surface area between the reacting phases.

- The extraction rate of divalent cobalt and its uptake by the organic phase is also increased in the presence of ultrasound. However, the sonochemical extraction of cobalt is characterized by a more difficult stripping of cobalt from the loaded organic phase. Because stripping of trivalent cobalt is always more difficult than extraction of divalent cobalt, it is believed that the ultrasonic treatment was responsible for the oxidation of cobalt during the sonochemical extraction.

- Even with the best conditions obtained in these studies, the energy consumption is still too high by a factor of $\sim 10$ when compared to the conventionally mixed solvent extraction process.

Further research is necessary to optimize the application of ultrasound in solvent extraction of metals. Special attention should be paid to the reactor design with respect to the following parameters; (a) sonochemical extraction under atmospheric versus pressurized conditions, (b) intermittent application of ultrasound, and (c) the application of ultrasound in a continuously flowing pipe mixer/reactor. 


\section{PART II: MECHANISMS ASSOCIATED WITH THE SOLVENT EXTRACTION OF GALLIUM USING KELEX 100 EXTRACTANT}

\section{Introduction}

The use of the extractant Kelex 100 for gallium recovery from sodium aluminate solutions used in the production of aluminum was initiated by Leveque and Helgorsky. ${ }^{48}$ They studied the effect of temperature and the effect of concentrations of a modifier and Kelex 100 on the recovery of gallium from the Pechiney Bayer circuit. They found that temperature had a positive effect on the solvent extraction of gallium in the range 20 to $75^{\circ} \mathrm{C}$. They chose $\mathrm{n}$-decanol as a modifier to prevent the formation of a third phase during stripping with hydrochloric acid.

Wang et al. ${ }^{49}$ studied the effect of temperature and the effects of sodium, aluminum, and Kelex 100 concentrations on the solvent extraction of gallium. They simulated the solvent extraction of gallium from sodium aluminate solutions that correspond to the sintering process leach solutions, which are far less concentrated in $\mathrm{Al}, \mathrm{Ga}$, and $\mathrm{Na}$ than the leach solutions produced in the Bayer process. They found that $\mathrm{Al}$ and $\mathrm{Na}$ had a negative effect on the solvent extraction of gallium.

Because the rate of the solvent extraction of gallium with Kelex 100 is very slow, efforts have been made to enhance the rate by the use of various surfactants. ${ }^{50,51 a, b}$. The enhanced rates of extraction observed with these surfactants were explained as being due to the reactions taking place at the interface between the aqueous phase and the microemulsions. ${ }^{52}$ In another approach, the original Kelex 100 molecule was modified to make the active sites more accessible to the gallate ions in the aqueous phase. 53 However, the new extractant, 5-substituted 8quinolinol, did not produce any improvement.

Sato and Oishi ${ }^{54}$ studied the solvent extraction of gallium from pure sodium hydroxide solutions. Kelex 100 dissolved in kerosene with no modifier was used. In the high sodium concentration range, the extraction rate of gallium was proportional to sodium and Kelex concentrations, and inversely proportional to hydroxide concentration. In the low concentration range, there was no effect of the hydroxide ion. To explain the effect of sodium, the formation of the $\mathrm{Na}^{+} \cdot \mathrm{Na}^{+}\left[\mathrm{Ga}(\mathrm{OH})_{3}\right]$ species was proposed as the rate controlling step. However, the solvent extraction of gallium from the sodium aluminate solutions was not studied, and, consequently, the importance of aluminum was not addressed. With respect to the mechanisms, it was presumed that the phenomena during gallium extraction from sodium hydroxide solutions were similar to those during the aluminum extraction from acid aqueous solutions with DEHPA. 55

The objective of the investigation presented in this part of the report was to examine the mechanisms involved during the solvent extraction of gallium with Kelex 100 from sodium aluminate solutions. Particular attention was focussed on the role of aluminum and sodium hydroxide in the aqueous phase. In the organic phase, ketone modifiers, having a high polar nature and rather strong donor character, were chosen to further elucidate the reaction mechanism. 


\section{Experimental Procedure}

The extractant, Kelex 100, was provided by Sherex Chemical Co. (Lot. No. 3349-118). The detailed chemical composition of Kelex 100 has been analyzed and published by Gareil et al. ${ }^{56}$ Diluents Kermac 470B (Triangle Refineries Co.) and Aromatic 150 (Exxon Co.) were used. Tributyl phosphate (TBP), decanol, isobutyl heptyl ketone (Union Carbide), and 2-undecanone were used as modifiers. Sodium hydroxide (A.R.) and aluminum hydroxide (A.R.) were obtained from the Baker Chemical Company. Sodium perchlorate monohydrate (A.R.) was obtained from EM Science. All these chemicals, except Kelex 100, were used without further purification.

Kelex 100 was purified to remove water soluble impurities. The chemical was contacted with $10 \% \mathrm{NaOH}$ by shaking, washed two times with $2 \mathrm{M} \mathrm{HCl}$, and then washed with deionized water until the $\mathrm{pH}$ was in the range 6 to 7 .

The experiments reported in this part of the report were performed with synthetic sodium aluminate solutions containing gallium. Gallium metal (gold label) was obtained from Aldrich Chemical Co. The stock solution of gallium was prepared by dissolving metallic gallium into nitric acid with some $\mathrm{HCl}$, heating to dryness to expel $\mathrm{N}_{2} \mathrm{O}$ and $\mathrm{HCl}$, and then diluting with sodium hydroxide solution.

A Perkin Elmer 2380 atomic absorption spectrophotometer was used to determine the gallium and aluminum concentrations in the aqueous phase. Sodium in the organic phase was first stripped with $1.5 \mathrm{M} \mathrm{HCl}$ and analyzed in with a Jenway PFP 7 flame photometer.

Raman spectra were obtained by using a Spex Model 14018 Ramalog monochromator with a home-built interface. A Spectra Physics 2025 Argon Ion Laser was used for excitation.

NMR spectra were obtained with an IBM NR-300 Spectrometer. The ${ }^{27} \mathrm{Al}$ NMR spectrum was produced at $78.21 \mathrm{MHz}$ by using an $\mathrm{AlCl}_{3}$ solution as the external reference. The ${ }^{71} \mathrm{Ga}$ spectrum was made at $91.53 \mathrm{MHz}$ by using a $\mathrm{Ga}\left(\mathrm{NO}_{3}\right)_{3}$ solution as the external reference.

The reactor used for the solvent extraction experiments was similar to that described in Part I of this report. Also, similar to the procedure used in Part I, the rate of solvent extraction of gallium was determined by analyzing the gallium concentration in the aqueous phase of the withdrawn and centrifuged samples by atomic absorption.

In the solvent extraction system, the organic phase consisted of $8 \%(\mathrm{v} / \mathrm{v})$ Kelex 100 extractant, $10 \%(\mathrm{v} / \mathrm{v})$ decanol (or other) modifier, and $82 \%(\mathrm{v} / \mathrm{v})$ Kermac $470 \mathrm{~B}$ diluent. The aqueous phase consisted of $225.8 \mathrm{~g} / \mathrm{L} \mathrm{NaOH}\left(175 \mathrm{~g} / \mathrm{L}\right.$ as $\left.\mathrm{Na}_{2} \mathrm{O}\right), 80 \mathrm{~g} / \mathrm{L} \mathrm{Al} \mathrm{O}_{3}$, and $0.20 \mathrm{~g} / \mathrm{L} \mathrm{Ga}$. The organic to aqueous (O/A) ratio was $1: 1$ (by volume), and the total volume in the reactor was $200 \mathrm{~mL}$. The standard temperature was $40^{\circ} \mathrm{C}$, and the stirring speed was $2000 \mathrm{rpm}$. 


\section{Results and Discussion}

\section{Effect of Stirring Speed}

Sodium aluminate solutions are very viscous and consequently require a large mixing energy input to achieve satisfactory extraction rates (within three hours). The effect of mixing was studied by varying the stirring speed in the range 1000 to $4000 \mathrm{rpm}$. Although the results are not given in this report, it was found that a stirring speed of $2000 \mathrm{rpm}$ was sufficient to eliminate bulk diffusion from controlling the rate of extraction of gallium. This stirring speed was used as a standard speed in subsequent experiments. It is interesting to note that Sato and Oishi ${ }^{54}$ performed their solvent extraction experiments in a mechanical shaker at $340 \mathrm{rpm}$, and found that this agitation was sufficient to eliminate bulk diffusion from controlling the rate of extraction.

\section{Effect of Temperature}

The effect of temperature on gallium extraction from a synthetic sodium aluminate solution was studied in the range 25 to $50^{\circ} \mathrm{C}$ and the results are presented in Figure 12. The rate of extraction increased with increased temperatures as indicated by the increased initial slopes for the curves for the higher temperatures. The rate of extraction at room temperature was slow, and equilibrium was not reached even after six hours of extraction. At $50^{\circ} \mathrm{C}$, the extraction rate was much faster, and it took only two hours to reach equilibrium. It is interesting to note that at equilibrium the amount of gallium extracted at $50^{\circ} \mathrm{C}$ was somewhat lower than at $40^{\circ} \mathrm{C}$. Leveque and Helgorsky ${ }^{48}$ had found that an increase in temperature, up to $75^{\circ} \mathrm{C}$, increased the extraction rate of gallium from the real solutions, but did not find that temperature had a significant effect on the amount of gallium extracted.

Similar to the procedure used in Part $I$, the apparent forward reaction rate constants were calculated according to Flett's method, ${ }^{40}$ from the slope of the line in a plot of $\left(C_{0}-C_{e}\right) / C_{0}$ $\ln \left[\left(C_{0}-C_{e}\right) /\left(C_{t}-C_{e}\right)\right]$ versus time. The reaction rate constants calculated from the data in Figure 12 are given in Table 8.

From an Arrhenius plot (Figure 13), the activation energy calculated from this data is $47.9 \mathrm{~kJ} / \mathrm{mole}$. This level of activation energy suggests a chemical reaction controlling mechanism. None of the previous studies on the solvent extraction of gallium reported activation energy values that could be used for comparison.

Table 8. Effect of temperature on the apparent forward reaction rate constant, $\mathrm{k}_{\mathrm{f}}$, during $\mathrm{Ga}$ extraction with Kelex 100 - Decanol - Kermac 470B.

\begin{tabular}{cc}
$\begin{array}{c}\text { Temperature } \\
\left({ }^{\circ} \mathrm{C}\right)\end{array}$ & $\begin{array}{c}\mathrm{k}_{\mathrm{f}} \times 10^{4} \\
\left(\mathrm{sec}^{-1}\right)\end{array}$ \\
\hline 25 & 1.04 \\
30 & 1.30 \\
40 & 2.86 \\
50 & 4.52 \\
\hline
\end{tabular}




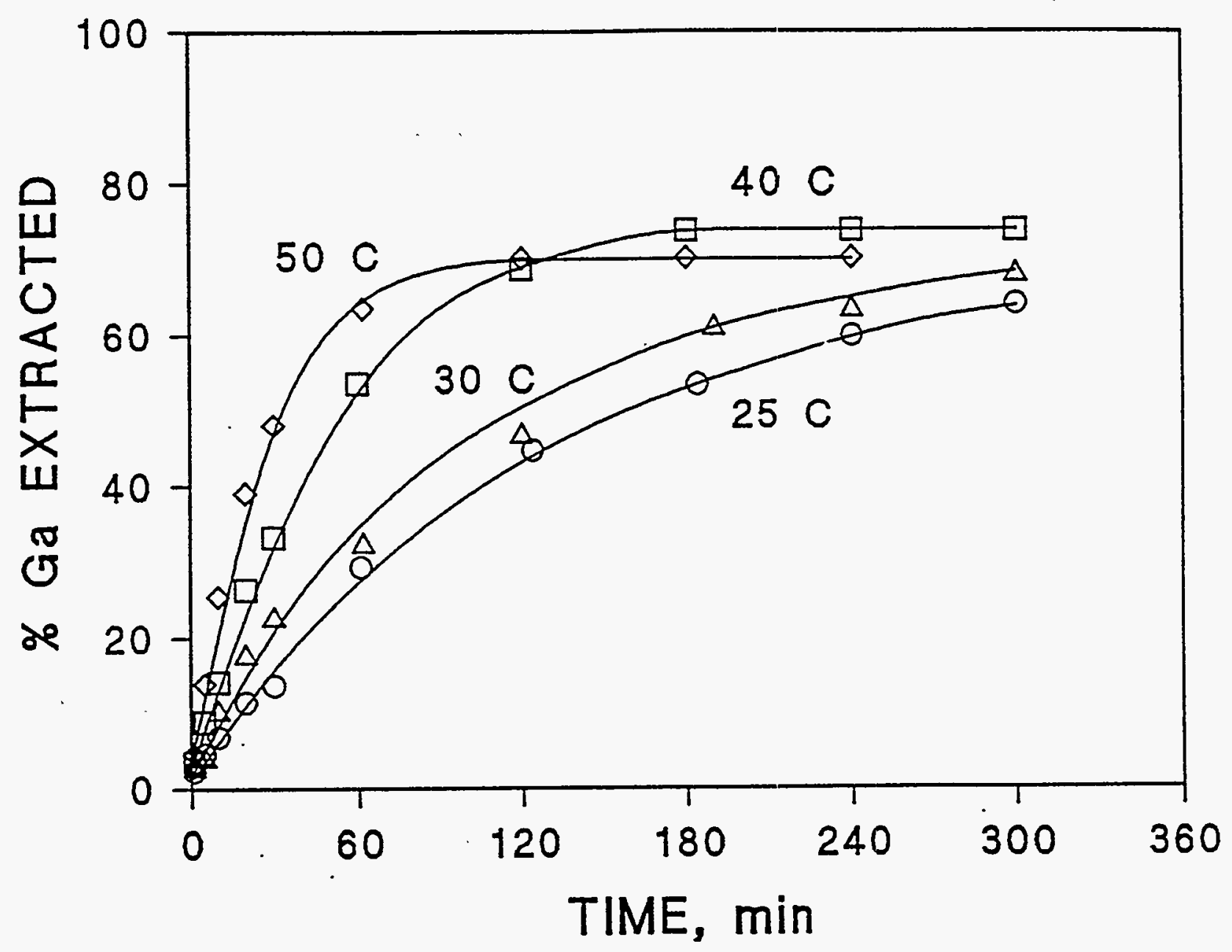

Figure 12. The effect of temperature on the solvent extraction of gallium with Kelex 100 decanol - Kermac 470B.

Organic phase, $(\mathrm{v} / \mathrm{v}): 8 \%$ Kelex 100, 10\% decanol, $82 \%$ Kermac $470 \mathrm{~B}$.

Aqueous phase, $(\mathrm{g} / \mathrm{L})$ : $80 \mathrm{Al}_{2} \mathrm{O}_{3}, 175 \mathrm{Na}_{2} \mathrm{O}, 0.2 \mathrm{Ga}$. 


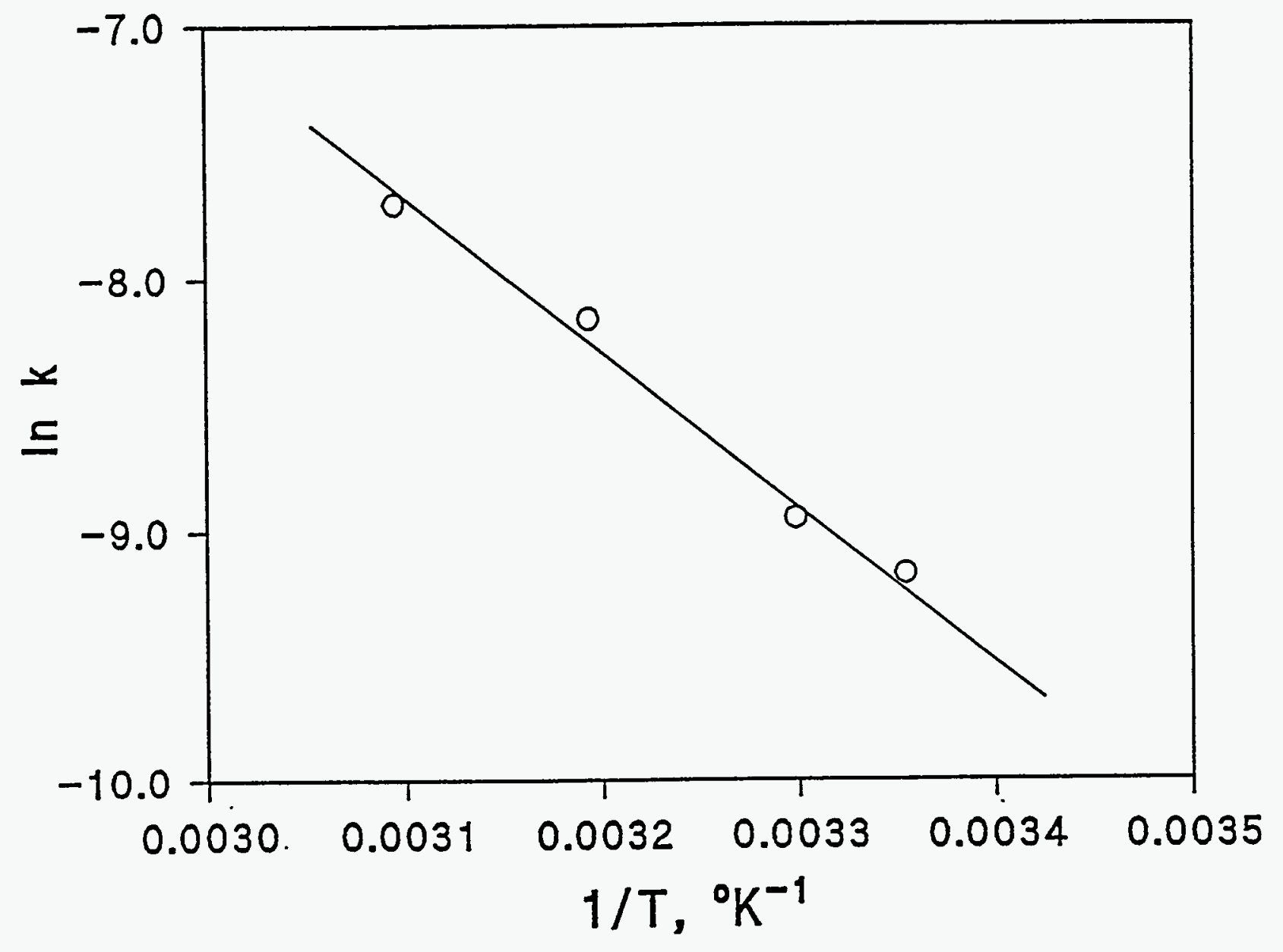

Figure 13. An Arrhenius plot for the solvent extraction of gallium with Kelex 100 - decanol Kermac 470B.

Organic phase, (v/v): $8 \%$ Kelex 100, 10\% decanol, 82\% Kermac 470B.

Aqueous phase, $(\mathrm{g} / \mathrm{L}): 80 \mathrm{Al}_{2} \mathrm{O}_{3}, 175 \mathrm{Na}_{2} \mathrm{O}, 0.2 \mathrm{Ga}$. 


\section{Effect of Modifiers}

Tributyl phosphate (TBP), decanol, isobutyl heptyl ketone, and 2-undecanone were used as modifiers. In general, it was found that ketones performed much better than alcohol and that, among the ketones, 2-undecanone performed best, as indicated in Figure 14. The effectiveness of the use of ketones (such as 2-undecanone) as modifiers for the solvent extraction of gallium has not been previously recognized.

The poor performance of the alcohol is explained as a result of the hydrogen bonding between the alcohol molecules and the $\mathrm{OH}$ groups of Kelex 100 molecules. This hydrogen bonding results in some of the active chelating sites of the Kelex molecules being occupied by the alcohol molecules, reducing the total number available for extraction.

The ketones, because of their high polar nature and rather strong donor character, will directly attract the gallate species, $\mathrm{Ga}(\mathrm{OH})_{4}$, toward the organic phase. The net effect of the attraction process is that water molecules remain behind (dehydration). This would be the first step in the overall process of solvent extraction. The second step would be the complexing of the sodium gallate species with the Kelex molecules.

\section{Effect of Aqueous Solution Composition}

The solvent extraction of gallium has been previously studied for the Kelex 100 - decanol Kermac $470 \mathrm{~B}$ system. ${ }^{57}$ The effect of sodium hydroxide concentration was investigated with the decanol system, (Figure 1 in Reference 57), and also here with the Kelex 100 - 2-undecanone Aromatic 150 system. The results obtained in this study are presented in Figure 15.

The results of both studies show that sodium hydroxide has a complex effect on solvent extraction of gallium. An increase of sodium hydroxide concentration in the solution results in an increase of the rate of solvent extraction of gallium, but only up to a certain concentration limit. Beyond this concentration limit, a further increase in concentration has a negative effect on the solvent extraction rate. The concentration limit is different for the different extraction systems. As indicated in Figure 16, the maximum extraction rate for the Kelex 100 - decanol - Kermac $470 \mathrm{~B}$ system was obtained at $151.6 \mathrm{~g} / \mathrm{L} \mathrm{Na}{ }_{2} \mathrm{O}(195.6 \mathrm{~g} / \mathrm{L} \mathrm{NaOH}, 2.4 \mathrm{~mol} / \mathrm{L}) .{ }^{57}$ As indicated in Figure 17, the maximum rate for the Kelex 100 - 2-undecanone - Aromatic 150 system corresponded to $210 \mathrm{~g} / \mathrm{L} \mathrm{Na} 2 \mathrm{O}(271 \mathrm{~g} / \mathrm{L} \mathrm{NaOH}, 3.4 \mathrm{~mol} / \mathrm{L})$. (Also shown in these figures are the rates associated with differing $\mathrm{K}_{2} \mathrm{O}$ concentrations, which will be discussed later.)

For both organic systems, it was also found that an increase in sodium hydroxide concentration has a negative effect on the equilibrium amount of gallium extracted. In the ketone system investigated here (Figure 15), the extraction line corresponding to $279 \mathrm{~g} / \mathrm{L} \mathrm{Na}_{2} \mathrm{O}$ is below the line corresponding to $245 \mathrm{~g} / \mathrm{L} \mathrm{Na} \mathrm{Na}_{2} \mathrm{O}$, which is below the line corresponding to $210 \mathrm{~g} / \mathrm{L}$ $\mathrm{Na}_{2} \mathrm{O}$. It might have been expected that the maximum gallium uptake should have been obtained for the lowest $\mathrm{Na}_{2} \mathrm{O}$ concentration, $102 \mathrm{~g} / \mathrm{L} \mathrm{Na}_{2} \mathrm{O}$. This was not the case, however, because the rate of gallium extraction was too slow at these lower sodium hydroxide concentrations to reach equilibrium during the time period monitored in the experiment. However, from examination of Figure 15, it is clear that the extraction was still proceeding at the lower sodium hydroxide concentrations. Similar arguments apply to the results obtained with the Kelex 100 - decanol Kermac 470 B system. ${ }^{57}$ 


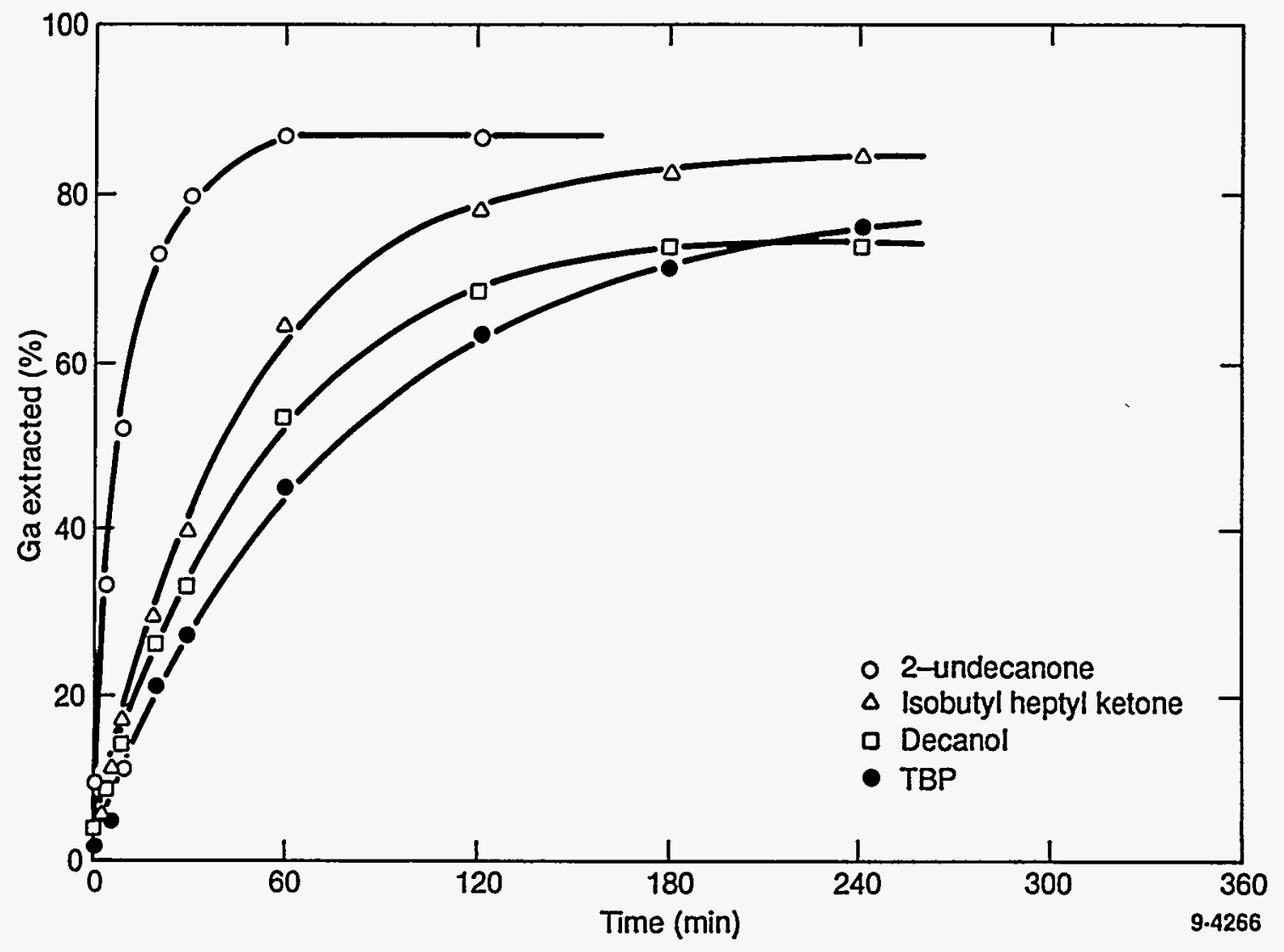

Figure 14. The effect of modifiers on the solvent extraction of gallium with Kelex 100 and Kermac 470B.

Organic phase, (v/v): $8 \%$ Kelex 100, 10\% modifier, and $82 \%$ Kermac $470 \mathrm{~B}$.

Aqueous phase, $(\mathrm{g} / \mathrm{L})$ : $175 \mathrm{Na}_{2} \mathrm{O}, 80 \mathrm{Al}_{2} \mathrm{O}_{3}$, and $0.2 \mathrm{Ga}$. 


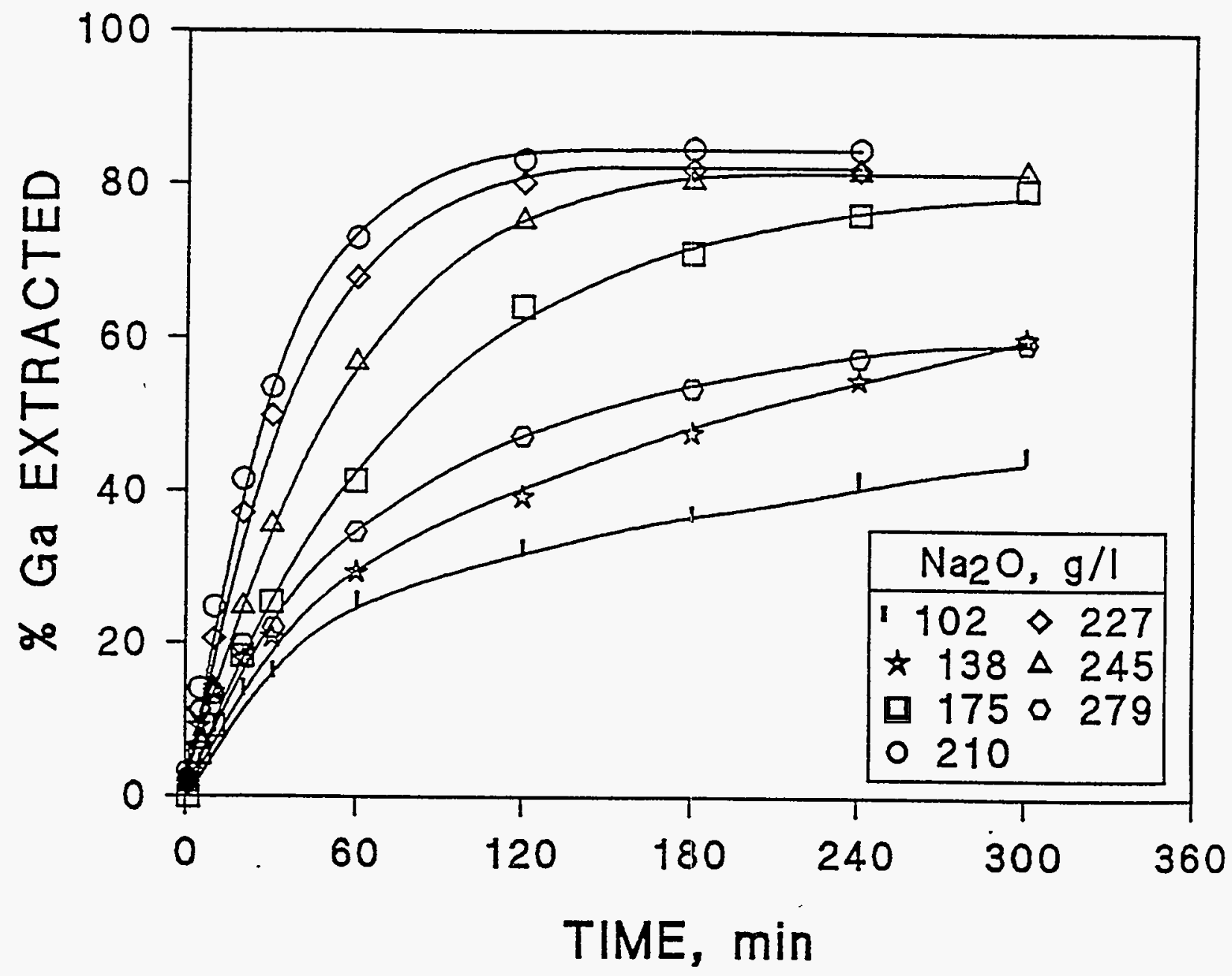

Figure 15. The extraction of gallium as a function of sodium hydroxide concentration. Organic phase, (v/v): $8 \%$ Kelex 100, 10\% 2-undecanone, 82\% Aromatic 150. Aqueous phase, $(\mathrm{g} / \mathrm{L}): 80 \mathrm{Al}_{2} \mathrm{O}_{3}$, indicated $\mathrm{Na}_{2} \mathrm{O}, 0.2 \mathrm{Ga}$. 


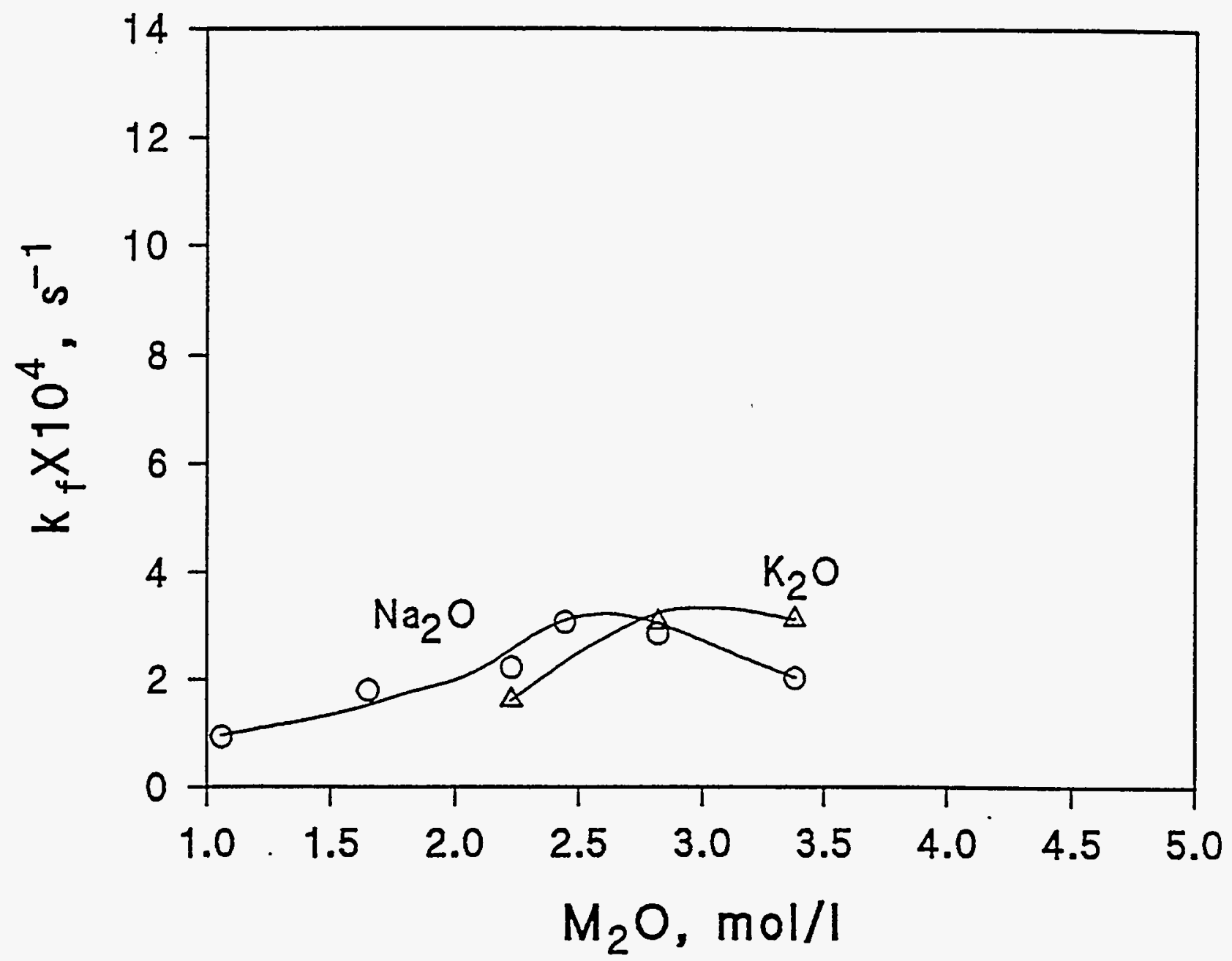

Figure 16. The effect of alkali concentration on the apparent forward reaction rate constant, $\mathrm{k}_{\mathrm{f}}$ for the extraction of gallium with Kelex 100 - decanol - Kermac 470B.

Organic phase, $(\mathrm{v} / \mathrm{v}): 8 \%$ Kelex 100, $10 \%$ decanol; 82\% Kermac 470B.

Aqueous phase, $(\mathrm{g} / \mathrm{L})$ : $80 \mathrm{Al}_{2} \mathrm{O}_{3}, 0.2 \mathrm{Ga}, \mathrm{Na}_{2} \mathrm{O}, \mathrm{K}_{2} 0$. 


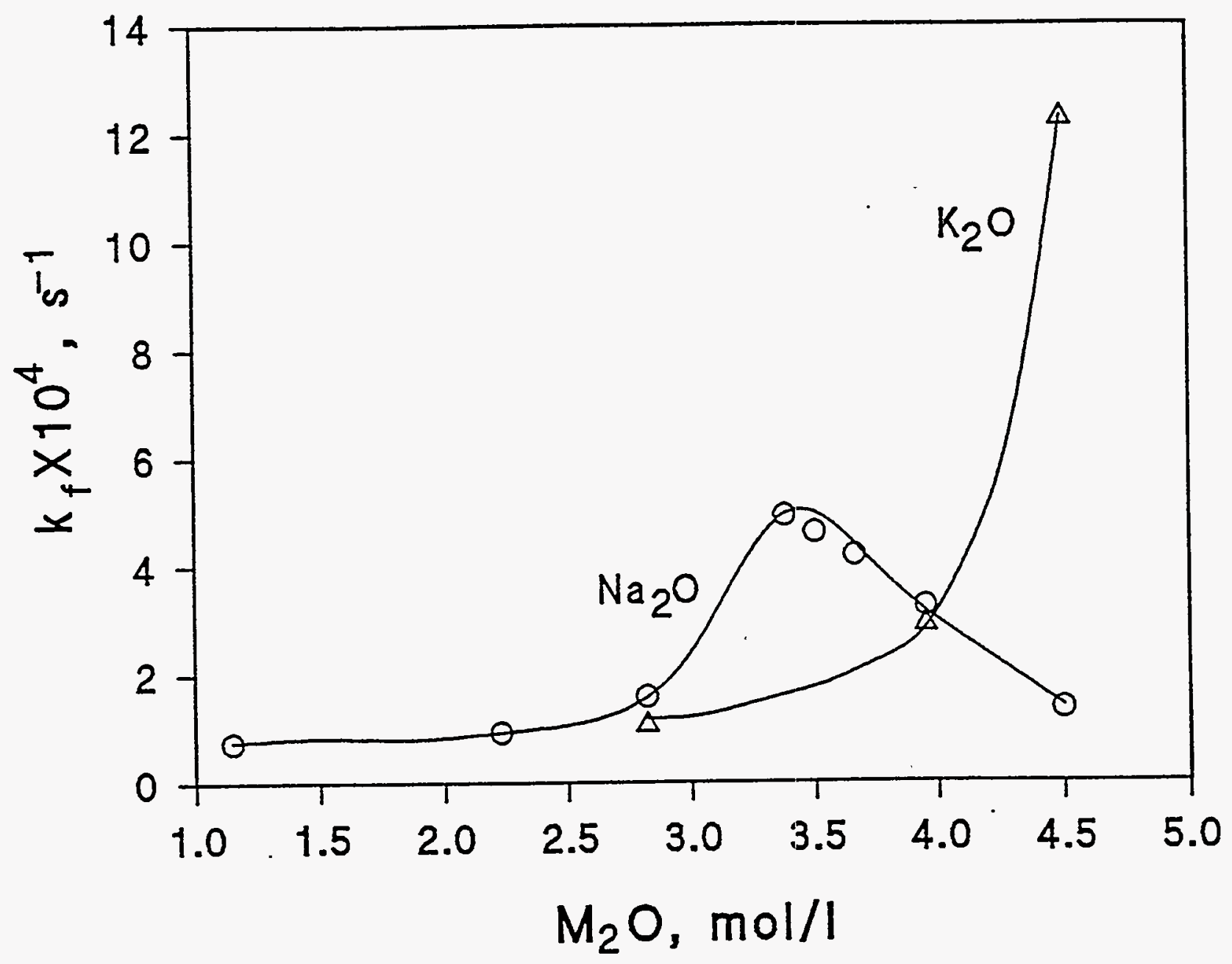

Figure 17. The effect of alkali concentration on the apparent forward reaction rate constant, $\mathrm{k}_{\mathrm{f}}$ for the extraction of gallium with Kelex 100 - 2-undecanone - Aromatic 150.

Organic phase, $(\mathrm{v} / \mathrm{v}): 8 \%$ Kelex 100, $10 \%$ 2-undecanone, $82 \%$ Aromatic 150.

Aqueous phase, $(\mathrm{g} / \mathrm{L})$ : $80 \mathrm{Al}_{2} \mathrm{O}_{3}, 0.2 \mathrm{Ga}, \mathrm{Na}_{2} \mathrm{O}, \mathrm{K}_{2} \mathrm{O}$. 
The negative effect of sodium hydroxide concentration on the extraction rate of gallium, beyond certain concentration thresholds, can be explained by the competitive extraction of sodium with Kelex 100. The negative effect was proven by analyzing the amount of sodium uptake into the organic phase as a function of the initial sodium hydroxide concentration in the aqueous phase. A logarithmic plot of equilibrium sodium concentration in the organic phase versus the initial sodium concentration in the aqueous phase is presented in Figure 18. It is clear from this figure that there is a sharply defined initial sodium hydroxide concentration at which sodium extraction becomes significant. This concentration exactly matches the concentrations found for the peak extraction rates shown in Figures 16 and 17, for the alcohol and ketone systems, respectively.

Gallium extraction as a function of aluminum hydroxide concentration was studied in the range of 0 to $132.5 \mathrm{~g} / \mathrm{L} \mathrm{Al}_{2} \mathrm{O}_{3}\left(202.8 \mathrm{~g} / \mathrm{L} \mathrm{Al}(\mathrm{OH})_{3}\right)$. Again, the Kelex 100 - decanol - Kermac 470B system has been previously studied. ${ }^{57}$ The results obtained for the Kelex 100 2-undecanone - Aromatic 150 system are presented in Figure 19. In both systems it was found that the aluminum had a negative effect on the rate and uptake of gallium at all concentration levels.

In the absence of aluminum, the extraction rate of gallium with the Kelex 100 2-undecanone - Aromatic 150 system was very fast, requiring only 30 minutes to reach equilibrium at about $98 \% \mathrm{Ga}$ extracted. In this case, with no aluminum, the performance of this system was much better than the performance of the Kelex 100 - decanol - Kermac 470B. ${ }^{57}$ When aluminum was added to the aqueous phase, the performance of both organic systems deteriorated, but the decrease in performance was greater for the Kelex 100 - 2-undecanone - Aromatic 150 system than for the Kelex 100 - decanol - Kermac 470B system. From plots of reaction rate versus aluminum hydroxide concentrations (not shown here), the reaction order was determined with respect to $\mathrm{Al}(\mathrm{OH})_{3}$ for the Kelex 100 - decanol - Kermac 470B system and the Kelex 100 2 -undecanone - Aromatic 150 system. The reaction orders were found to be -0.4 and -1.0 for these two systems, respectively.

\section{Extraction Mechanism}

The extraction of gallium with Kelex 100 from sodium aluminate solutions consists of several steps. Gallium in the sodium aluminate solution is present as the monomeric gallate ion, $\mathrm{Ga}(\mathrm{OH})_{4}^{-}$, and aluminum is present as the monomeric aluminate ion, $\mathrm{Al}(\mathrm{OH})_{4}^{-}$. The $\mathrm{T}_{\mathrm{d}}$ symmetry of the Raman spectra has indicated that four hydroxide ions surround the metal cations in the form of a tetrahedron. ${ }^{58,59}$ This has also been confirmed in this study. No dimerization of the gallate or aluminate ions was found in the solutions studied here, as shown by the sharp ${ }^{71} \mathrm{Ga}$ and ${ }^{27} \mathrm{Al} \mathrm{NMR}$ peaks in Figure 20. According to Moolenaar, Evans, and McKeever, ${ }^{58}$ the dimerization of the aluminate ions takes place only at high concentrations of alumina, above $80 \mathrm{~g} / \mathrm{L} \mathrm{Al} \mathrm{O}_{3}$.

The first reaction step involves dehydration followed by the release of the first $\mathrm{OH}^{-}$group from the gallate, within the closely spaced, hydrated, sodium and gallate ions at the interface as follows: 


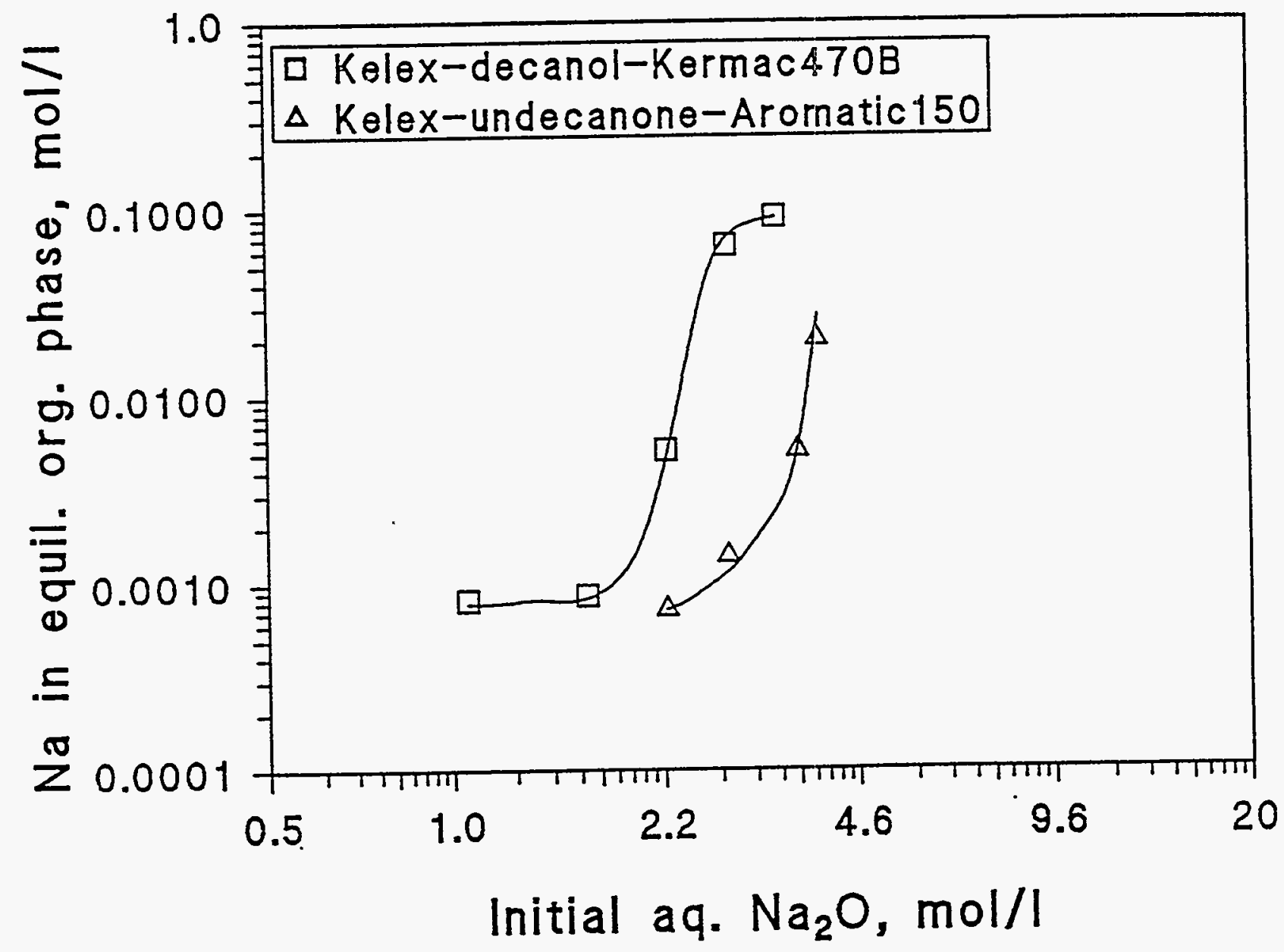

Figure 18. The extraction of sodium into the organic phase as a function of the initial aqueous sodium hydroxide concentration during the solvent extraction of gallium. 


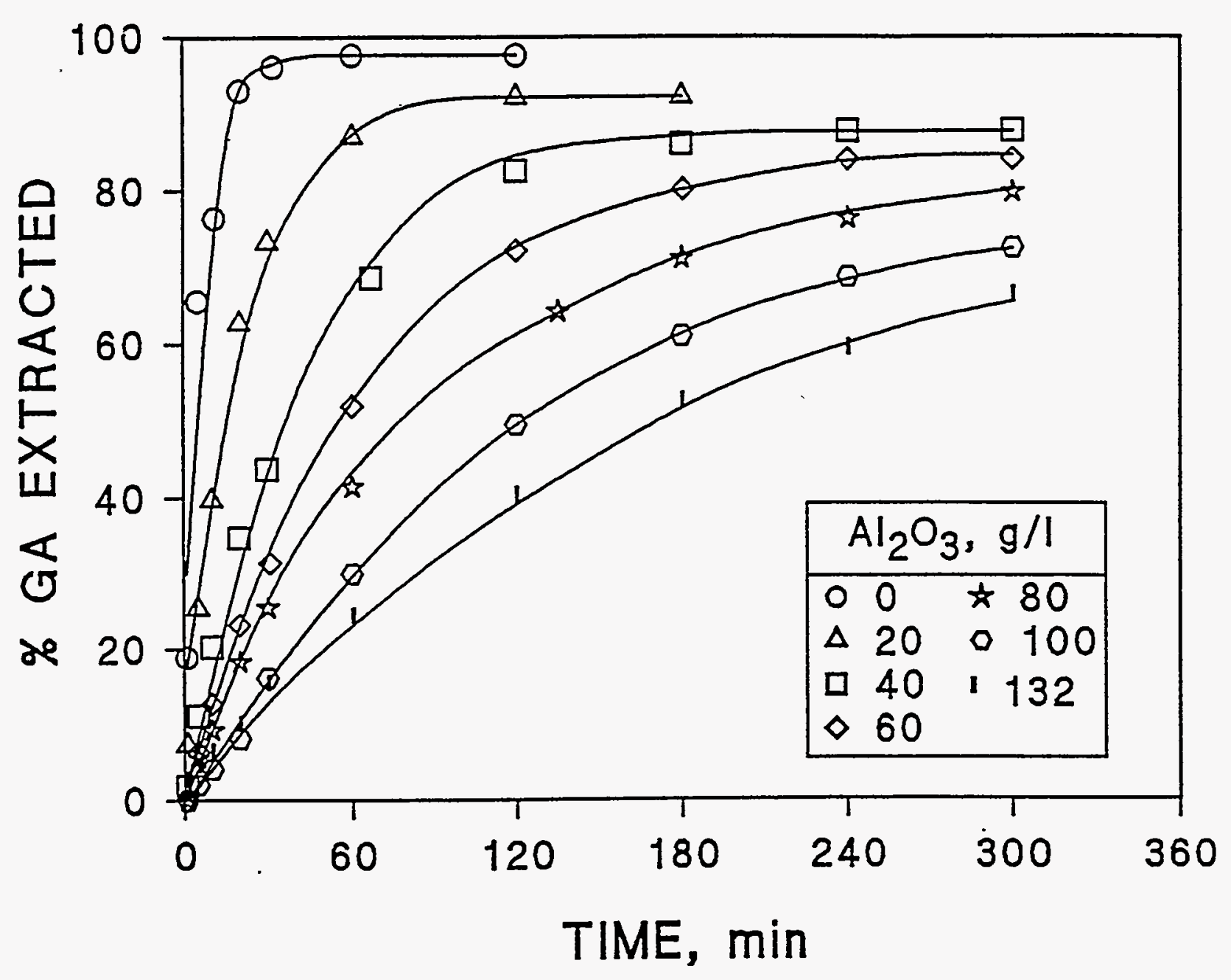

Figure 19. Solvent extraction of gallium as a function of alumina concentration.

Organic phase, (v/v): $8 \%$ Kelex 100, 10\% 2-undecanone, 82\% Aromatic 150.

Aqueous phase, $(\mathrm{g} / \mathrm{L})$ : Indicated $\mathrm{Al}_{2} \mathrm{O}_{3}, 175 \mathrm{Na}_{2} \mathrm{O}, 0.2 \mathrm{Ga}$. 


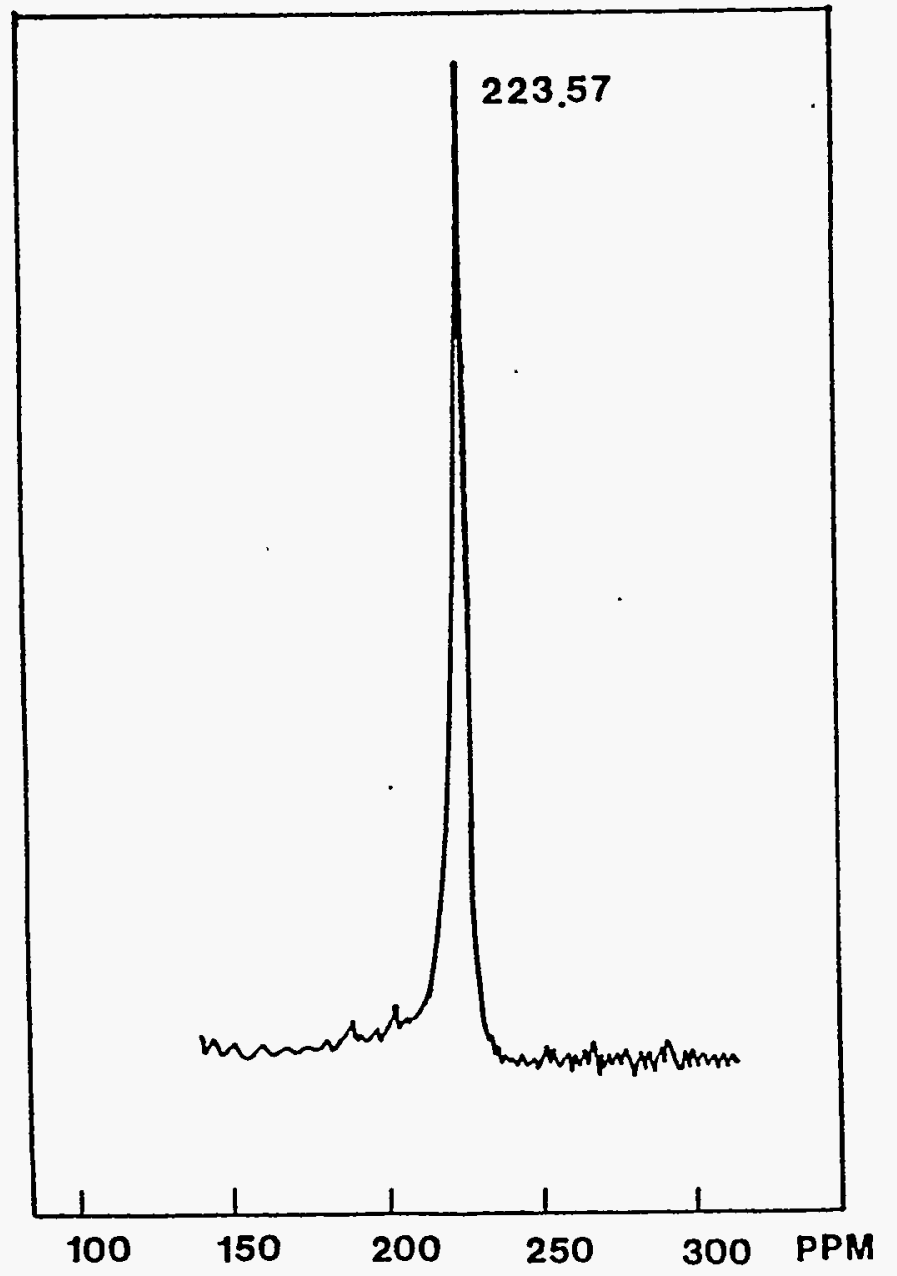

(a)

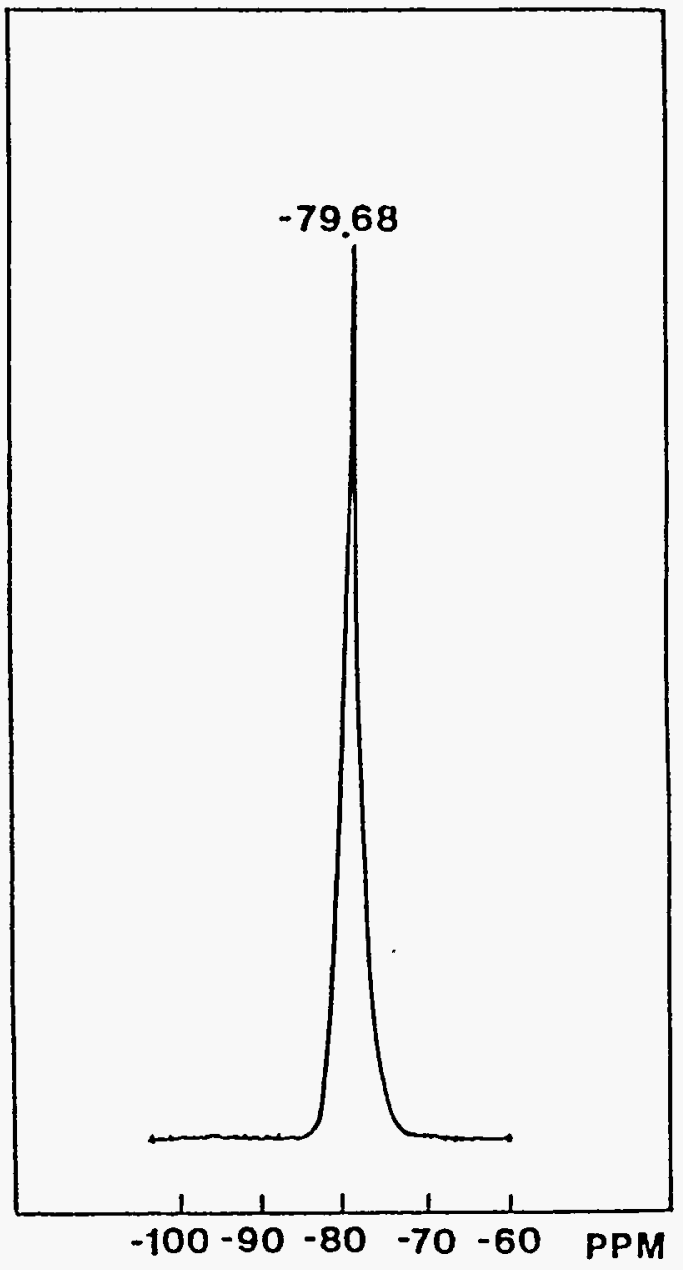

(b)

Figure 20. NMR spectra of ${ }^{71} \mathrm{Ga}$ and ${ }^{27} \mathrm{Al}$ for the aqueous solutions:

(a) $11.0 \mathrm{~g} / \mathrm{L} \mathrm{Ga}, 175 \mathrm{~g} / \mathrm{L} \mathrm{Na} 2 \mathrm{O}$, no aluminum

(b) $80 \mathrm{~g} / \mathrm{L} \mathrm{Al}_{2} \mathrm{O}_{3}, 175 \mathrm{~g} / \mathrm{L} \mathrm{Na} \mathrm{Na}_{2}$, no gallium. 


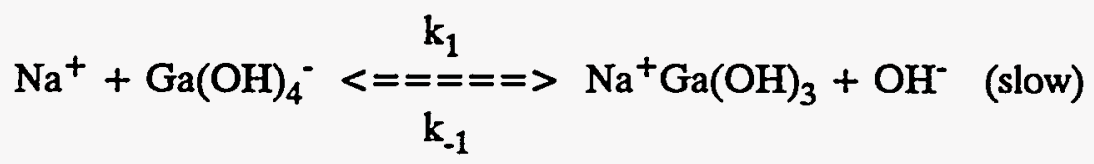

where $\mathrm{k}_{1}$ is the forward reaction rate constant corresponding to the dehydration and the release of the first $\mathrm{OH}^{-}$group from the gallate, and $\mathrm{k}_{-1}$ is the back reaction rate constant.

The water shells surrounding the gallate and the aluminate ions are extremely stable, ${ }^{60}$ and the rate of removal of water molecules from $\mathrm{Ga}^{3+}$ is very slow, much slower than the rate of hydrolysis. ${ }^{61}$ The number of water molecules in the shells surrounding the gallate and the aluminate ions, estimated by Dovbysh, Volokhov, Eremin, and Mironov, ${ }^{60}$ greatly depends on the solution composition. Thus, when increasing the concentration of sodium, the positive charge of the sodium ions will cause some of the water molecules from the water shell surrounding the gallate ions to move into the water shell surrounding the sodium ions. This dehydration phenomenon will make room for the hydroxide ions to leave the gallate ions by Reaction (1). The increase of sodium concentration, therefore, should increase the gallium extraction rate.

Further evidence of the importance of dehydration on the extraction rate of gallium is related to the solution composition. By using a larger cation, as a substitute for $\mathrm{Na}^{+}$in the $\mathrm{Na}^{+}$ $+\mathrm{Ga}(\mathrm{OH})_{4}{ }^{-}$in Reaction (1), it was expected that the dehydration of the gallate ion water shell would be less pronounced, because of the smaller charge density of the larger cation. The extraction rates of gallium were indeed smaller in the presence of potassium as shown in Figure 16. By increasing the concentration of potassium, the rate increased, as in the case with sodium, until a certain potassium concentration was reached, and then the extraction rate began to decrease. The size of the peaks for potassium was about the same as for sodium. For the same concentration of potassium and sodium, the rate of extraction would be smaller in the presence of potassium for those concentrations to the left of the intersection point. In the case of the Kelex 100 - 2-undecanone - Aromatic 150 system (Figure 17), the intersection point for the rates was not achieved until much larger concentrations, $4.0 \mathrm{~mol} / \mathrm{L} \mathrm{M}_{2} \mathrm{O}$, above which the rate in the potassium aluminate solutions was larger than in the sodium aluminate solutions. A concentration of $\mathrm{K}_{2} \mathrm{O}$ higher than $4.5 \mathrm{~mol} / \mathrm{L}(424 \mathrm{~g} / \mathrm{L})$ was not studied because of solubility problems.

According to the $\mathrm{S}_{\mathrm{N}} 1$ mechanism, the $\mathrm{OH}^{-}$leaving group would be subsequently replaced by the entering Kelex 100 molecules at the interface:

$$
\mathrm{Na}^{+} \mathrm{Ga}(\mathrm{OH})_{3 \text { aq }}+\mathrm{HQ}_{\text {org }} \stackrel{\mathrm{k}_{2}}{--\cdot>} \mathrm{Na}^{+}+\mathrm{GaQ}(\mathrm{OH})_{2 \text { org }}+\mathrm{H}_{2} \mathrm{O} \text { (slow) }
$$

where $k_{2}$ is the first chelation rate constant of HQ (Kelex 100), with gallium.

The final step, which is a fast step, is the completion of chelation of gallium with Kelex 100:

$$
\mathrm{GaQ}(\mathrm{OH})_{2 \text { org }}+2 \mathrm{HQ}_{\text {org }} \stackrel{\mathrm{k}_{\mathrm{e}}}{---->\mathrm{GaQ}_{3} \text { org }}+2 \mathrm{H}_{2} \mathrm{O} \text { (fast) }
$$


The rate of Reaction (1) can be expressed in terms of the rate of production of $\mathrm{Na}^{+} \mathrm{Ga}(\mathrm{OH})_{3}$ :

$$
\begin{aligned}
& \mathrm{d}\left[\mathrm{Na}^{+} \mathrm{Ga}(\mathrm{OH})_{3}\right] / \mathrm{dt} \\
& =\mathrm{k}_{1}\left[\mathrm{Na}^{+}\right]\left[\mathrm{Ga}(\mathrm{OH})_{4}^{-}\right]-\mathrm{k}_{-1}\left[\mathrm{Na}^{+} \mathrm{Ga}(\mathrm{OH})_{3}\right]\left[\mathrm{OH}^{-}\right]-\mathrm{k}_{2}\left[\mathrm{Na}^{+} \mathrm{Ga}(\mathrm{OH})_{3}\right][\mathrm{HQ}] \\
& =\mathrm{k}_{1}\left[\mathrm{Na}^{+}\right]\left[\mathrm{Ga}(\mathrm{OH})_{4}^{-}\right]-\left(\mathrm{k}_{-1}\left[\mathrm{OH}^{-}\right]+\mathrm{k}_{2}[\mathrm{HQ}]\right)\left[\mathrm{Na}^{+} \mathrm{Ga}(\mathrm{OH})_{3}\right] \\
& \mathrm{d}\left[\mathrm{GaQ}_{3}\right] / \mathrm{dt}=\mathrm{K}^{\prime} \mathrm{d}\left[\mathrm{GaQ}(\mathrm{OH})_{2}\right] / \mathrm{dt}=\mathrm{K}^{\prime} \mathrm{k}_{2}\left[\mathrm{Na}^{+} \mathrm{Ga}(\mathrm{OH})_{3}\right][\mathrm{HQ}]
\end{aligned}
$$

where $\mathrm{K}^{\prime}=\mathrm{k}_{\mathrm{e}}[\mathrm{HQ}]^{2}, \mathrm{k}_{\mathrm{e}}$ is the equilibrium constant of Reaction (3). At steady state:

$$
\mathrm{d}\left[\mathrm{GaQ}_{3}\right] / \mathrm{dt}=\mathrm{K}^{\prime} \mathrm{k}_{1} \mathrm{k}_{2}\left[\mathrm{Na}^{+}\right]\left[\mathrm{Ga}(\mathrm{OH})_{4}{ }^{-}\right][\mathrm{HQ}] /\left(\mathrm{k}_{-1}\left[\mathrm{OH}^{-}\right]+\mathrm{k}_{2}[\mathrm{HQ}]\right)
$$

and if $\mathrm{k}_{-1}\left[\mathrm{OH}^{-}\right]>>\mathrm{k}_{2}[\mathrm{HQ}]$, then Equation (6) becomes:

$$
\mathrm{d}\left[\mathrm{GaQ}_{3}\right] / \mathrm{dt}=\mathrm{K}^{\prime} \mathrm{k}_{1} \mathrm{k}_{2}\left[\mathrm{Na}^{+}\right]\left[\mathrm{Ga}(\mathrm{OH})_{4}^{-}\right][\mathrm{HQ}] / \mathrm{k}_{-1}\left[\mathrm{OH}^{-}\right]
$$

Equation (7) represents the rate of solvent extraction of gallium with Kelex 100. According to this expression, the rate of gallium extraction should be first order with respect to gallium, sodium, and Kelex 100, and inversely proportional to the $\mathrm{OH}^{-}$concentration. The first order reaction with respect to gallium was already proved experimentally by fitting Flett's model, ${ }^{40}$ which was derived for a first order reaction. With respect to sodium, the reaction order was determined by using sodium perchlorate to vary the sodium concentration, and it was found that a first order reaction was obtained. With respect to $\mathrm{OH}^{-}$, the reaction order was minus one. With respect to Kelex 100, a fractional reaction order, 0.5 , was obtained. (The data to determine these reaction orders have not been presented in this report.) The reaction order obtained for the Kelex 100 is not in accordance with the predicted value of 1, as indicated by Equation (7), because the extraction rate of gallium is governed by the slow steps Reactions (1) and (2) that only reflect the concentration of Kelex 100 at the interface, which apparently was different from the concentration in the bulk.

Equation (7) does not explicitly predict the negative effect of aluminum. Instead, the effect of aluminum is contained in the constant $k_{1}$. The influence of aluminum can be explained by its negative effect on dehydration. The support for this conclusion can also be found by analyzing the theoretical rates of water substitution from the inner coordinating sphere for metals. ${ }^{61}$ Accordingly, the specific rate constant for water substitution in the inner coordination sphere for aluminum is about 10 times smaller than for gallium. Therefore, the addition of aluminum hydroxide to the gallate-containing solutions will cause the stabilization of the water shell in the gallate structure.

An additional diagnostic tool that can be used to support the role of dehydration is the comparison of experimental dehydration activation energies. By using the $\mathrm{H}$ spin-lattice relaxation time and the model by Hindman, ${ }^{62}$ the activation energy for translation and rotation of water molecules around the gallate ions was calculated. It was found that the activation energy to remove an individual water molecule was $18.9 \mathrm{~kJ} / \mathrm{mole}$, while the activation energy to remove the water molecules during hydrogen bond breakage (small clusters) required $43.9 \mathrm{~kJ} / \mathrm{mole}{ }^{63}$ The 
experimentally determined activation energy ( $47.9 \mathrm{~kJ} / \mathrm{mole})$ is very close to the calculated values and supports the proposed dehydration mechanism.

At very high aluminum concentrations, the dimerized aluminates may also contribute to the slow extraction rate of gallium. That the negative effect of aluminum was not caused by the competitive extraction was proven experimentally. In four tests, the organic phase was contacted with the sodium aluminate solutions (standard conditions) for $0,1,4$, and 24 hours before gallium was added to the solution. Identical solvent extraction rate curves were obtained, which proved that the uptake of aluminum by Kelex 100 was minimal and without any effect. The analysis of the organic phase showed that only about $1 \%$ of aluminum was extracted by the organic phase during the $31 \mathrm{~h}$ ( $24 \mathrm{~h}$ pretreatment $+7 \mathrm{~h}$ extraction) contact time. Sato, ${ }^{59}$ in an experiment on the extraction of aluminum (in the absence of gallium) with Kelex 100, also showed that the rate of aluminum extraction was extremely slow. However, the amount of aluminum extracted by Sato was much higher, $\sim 45 \%$, because the concentration of $\mathrm{NaOH}$ was much lower $(1 M)$, than in this case $(5.6 M)$.

A similar reaction mechanism applies to the Kelex 100 - 2-undecanone - Aromatic 150 system with a slight difference in the dehydration reaction path. The coordination of the donor ketone with gallium may also be responsible for the substitution of water molecules in the shell of the gallate ions, causing significantly increased rates of gallium extraction.

\section{Conclusions for Part II}

On the basis of the experiments described in Part II of this report, it is clear that the solvent extraction of gallium from sodium aluminate solutions is a very complex phenomenon. The rate of extraction is a function of the composition of aqueous solution (sodium, aluminum, and hydroxide) and the nature of the organic phase. On the basis of these experiments the following conclusions have been drawn:

- The rate of gallium extraction can be represented by the equation

$$
\mathrm{d}\left[\mathrm{GaQ}_{3}\right] / \mathrm{dt}=\mathrm{K}^{\prime} \mathrm{k}_{1} \mathrm{k}_{2}\left[\mathrm{Na}^{+}\right]\left[\mathrm{Ga}(\mathrm{OH})_{4}{ }^{-}\right][\mathrm{HQ}] / \mathrm{k}_{-1}\left[\mathrm{OH}^{-}\right]
$$

where $\mathrm{k}_{1}$ also includes the effects of sodium hydroxide and aluminum hydroxide. The rate controlling step is the dehydration of the gallate ion, $\mathrm{Ga}(\mathrm{OH})_{4}{ }^{-}$, and the first complex formation between gallium and Kelex 100.

- Sodium influences the solvent extraction of gallium through its effect on dehydration and by the competitive coextraction with Kelex 100 . Aluminum influences the solvent extraction of gallium only through its effects on dehydration ( $k_{1}$ constant).

- Sodium hydroxide has a positive effect on the extraction rate of gallium up to $195.6 \mathrm{~g} / \mathrm{L}$ $\mathrm{NaOH}$ (for the Kelex 100 - decanol - Kermac 470B system), and up to $271 \mathrm{~g} / \mathrm{L} \mathrm{NaOH}$ (for the Kelex 100 - undecanone - Aromatic 150 system), at which concentrations the coextraction of sodium becomes significant. Beyond these values, increasing sodium hydroxide concentration has a negative effect. 
- Hydroxide ion has a negative effect on the solvent extraction of gallium from sodium aluminate solutions.

- The activation energy for the solvent extraction of gallium with Kelex 100 - decanol Kermac $470 \mathrm{~B}$ was found to be $47.9 \mathrm{~kJ} / \mathrm{mole}$, and is consistent with the proposed dehydration mechanism.

- Because of their high polar nature and rather strong donor character, ketone modifiers (particularly 2-undecanone), enhance the dehydration of gallate ions and, consequently, the rates of gallium extraction. 


\section{PART III: THE EFFECT OF ULTRASOUND ON THE SOLVENT EXTRACTION OF GALLIUM}

\section{Introduction}

Gallium can be recovered by solvent extraction from leach liquors used in the production of aluminum. The leach liquors from the Bayer process for the manufacture of aluminum contain a significant amount of gallium, typically 0.1 to $0.15 \mathrm{~g} / \mathrm{L}$. The major components of the leach solution are aluminum oxide $(80$ to $120 \mathrm{~g} / \mathrm{L}$ ) and sodium hydroxide $(140$ to $210 \mathrm{~g} / \mathrm{L})$. Although Kelex 100 can extract gallium from the sodium aluminate solutions, the kinetics are very slow, and gallium is not currently recovered in the U.S.

Various attempts have been made to increase the solvent extraction rate of gallium. The major conceptual approach has been to increase the interfacial surface area by adding surfactants; versatic acid, ${ }^{50}$ organic sulfonate, ${ }^{51 a}$ or acidic organic phosphoric compounds ${ }^{51 b}$ have been tried. Extraction rates increased remarkably when decanol was used as a cosurfactant. ${ }^{45,64}$ This effect was attributed to the production of microemulsions, which greatly increased the interfacial surface area between the aqueous and organic phases of the system.

In Part II of this report, the mechanisms involved in gallium solvent extraction with Kelex 100 were discussed. The extraction rate of gallium is very slow and was found to be controlled by the dehydration of gallate ions (the removal of water molecules from the water shell surrounding the sodium and gallate ions, permitting a hydroxide ion to leave the gallate ion). The cavitation effects produced by ultrasound would be expected to increase the rate of gallium solvent extraction by either increasing the rate of dehydration or by increasing the interfacial surface area between the two immiscible phases. If the use of ultrasound could significantly increase the rate of reaction, the solvent recovery of gallium from these leach liquors could be more economical. The results of the investigation of the effect of ultrasound on the solvent extraction of gallium using Kelex 100 is presented in this part of the report.

\section{Experimental Procedure}

The extractant, Kelex 100, was provided by Sherex Chemical Co. (Lot. No. 3349-118).

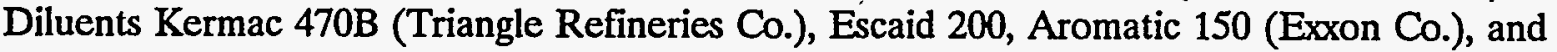
cyclohexane (EM Science) were used. Decanol and 2-undecanone (Aldrich Chem. Co.) were used as modifiers. All these chemicals, except Kelex 100, were used without further purification.

Kelex 100 was purified to remove water soluble impurities. The chemical was contacted with $10 \% \mathrm{NaOH}$ by shaking, washed two times with $2 \mathrm{M} \mathrm{HCl}$, and then washed with deionized water until the $\mathrm{pH}$ was in the range 6 to 7 .

The first set of experiments reported in this part of the report were performed with synthetic sodium aluminate solutions containing gallium. Gallium metal (gold label) was obtained from Aldrich Chemical Co. The stock solution of gallium was prepared by dissolving metallic gallium into nitric acid with some $\mathrm{HCl}$, heating to dryness to expel $\mathrm{N}_{2} \mathrm{O}$ and $\mathrm{HCl}$, and then diluting with sodium hydroxide solution. Sodium hydroxide (A.R.) and aluminum hydroxide 
(A.R.) were obtained from Baker Chemical Company. For the second set of experiments, an industrial process sodium aluminate solution was obtained from Kaiser Aluminum Co. (Louisiana).

The reactor used for the solvent extraction experiments was similar to that described in Part I of this report, except that the ultrasound was produced with a Heat Systems Model W-220F ultrasonic generator. The frequency of ultrasound was $20 \mathrm{kHz}$, and the ultrasonic power input was $47 \mathrm{~W}$.

Also, similar to the procedure used in Part I, the rate of solvent extraction of gallium was determined by analyzing the gallium concentration in the aqueous phase of the withdrawn and centrifuged samples by atomic absorption.

In the solvent extraction system, the organic phase consisted of $8 \%(\mathrm{v} / \mathrm{v})$ Kelex 100 extractant, $10 \%(\mathrm{v} / \mathrm{v})$ modifier, and $82 \%(\mathrm{v} / \mathrm{v})$ diluent. The aqueous phase consisted of $225.8 \mathrm{~g} / \mathrm{L}$ $\mathrm{NaOH}\left(175 \mathrm{~g} / \mathrm{L}\right.$ as $\left.\mathrm{Na}_{2} \mathrm{O}\right), 80 \mathrm{~g} / \mathrm{L} \mathrm{Al}_{2} \mathrm{O}_{3}$, and $0.20 \mathrm{~g} / \mathrm{L} \mathrm{Ga}$. The O/A ratio was 1:1, and the total volume in the reactor was $200 \mathrm{~mL}$. The standard temperature was $40^{\circ} \mathrm{C}$, and stirring speed was $2000 \mathrm{rpm}$.

\section{Sonochemical Extraction of Gallium from Synthetic Sodium Aluminate Solutions}

\section{Effect of Stirring Speed}

The solvent extraction of gallium with Kelex 100 is very slow and energy intensive. As shown in Figure 21, increasing the stirring speed increases the rate of solvent extraction, but no rate plateau was obtained in the range 1000 to $4000 \mathrm{rpm}$ without ultrasound. At $2000 \mathrm{rpm}$, it took more than $3 \mathrm{~h}$ to reach equilibrium. When ultrasound was introduced, mechanical stirring was not an important parameter. The presence of ultrasound results in a much faster gallium extraction rate, and equilibrium was reached after only 30 min. During the ultrasonic treatment, the whole reactor was filled with tiny droplets, and the solution system looked like an emulsion. When the ultrasonic treatment was stopped, the phases separated quickly, within $5 \mathrm{~min}$. The ultrasound application did not produce stable emulsions in this system.

\section{Effect of Temperature}

The effects of temperature were studied in the range 30 to $50^{\circ} \mathrm{C}$ as indicated in Figure 22 . In the absence of ultrasound, the temperature had a positive effect on the extraction rates. It is interesting to note that when ultrasound was used, there was no effect of temperature on the solvent extraction rates of gallium. Similar to the procedure used in Parts I and II, the apparent forward reaction rate constants, $k_{f}$, were calculated for each extraction experiment according to Flett's method, ${ }^{40}$ These constants, calculated from the data shown in Figure 22, are presented in Table 9. 


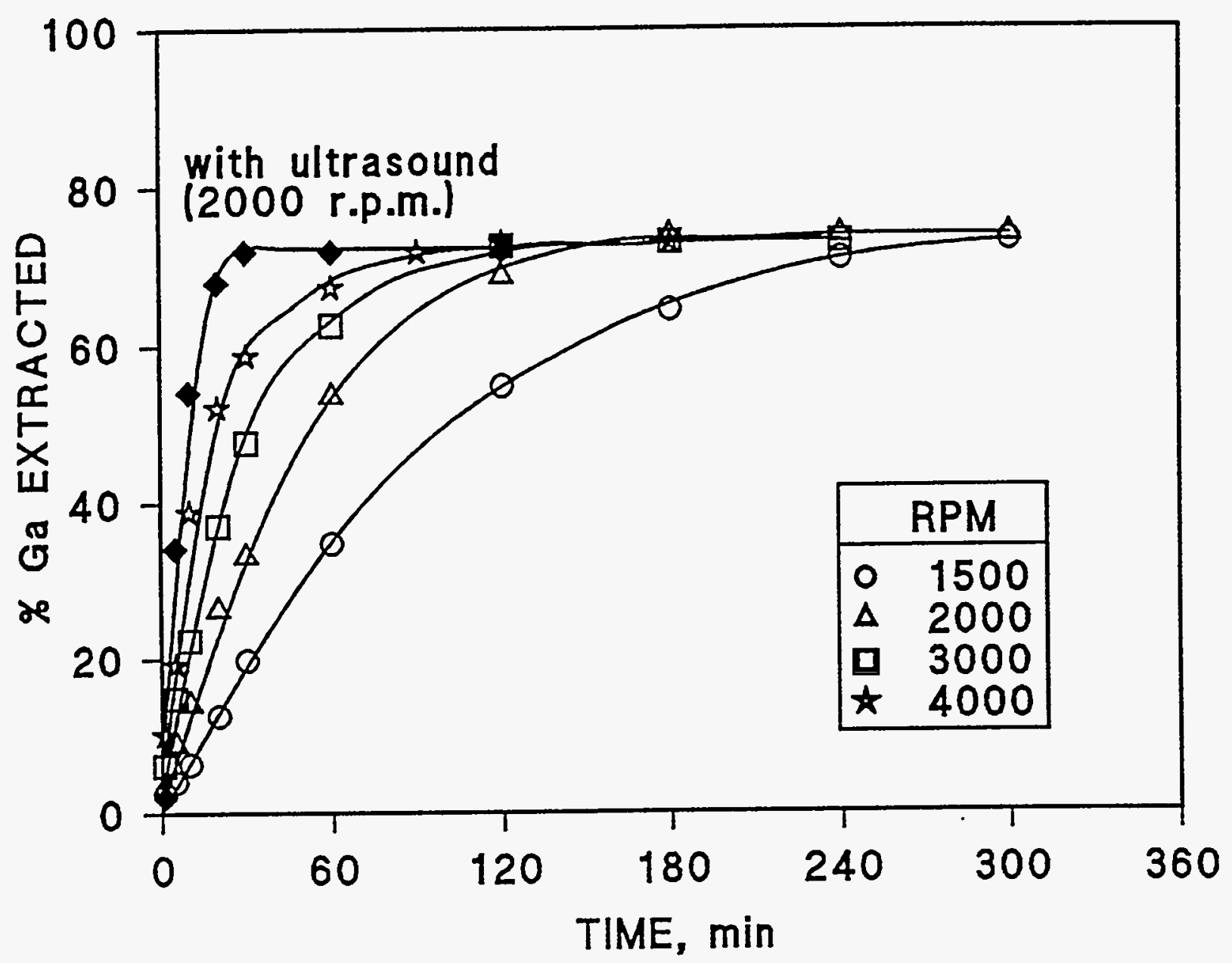

Figure 21. The effect of stirring speed on the solvent extraction of gallium with Kelex 100 decanol - Kermac 470B.

Organic phase, (v/v): $8 \%$ Kelex 100, 10\% decanol, $82 \%$ Kermac 470B.

Aqueous phase, $(\mathrm{g} / \mathrm{L})$ : $0.2 \mathrm{Ga}, 175 \mathrm{Na}_{2} \mathrm{O}, 80 \mathrm{Al}_{2} \mathrm{O}_{3}$.

System: $\mathrm{O} / \mathrm{A}=1: 1, T=40^{\circ} \mathrm{C}$.

Ultrasound: $20 \mathrm{kHz}, 47 \mathrm{~W}$. 


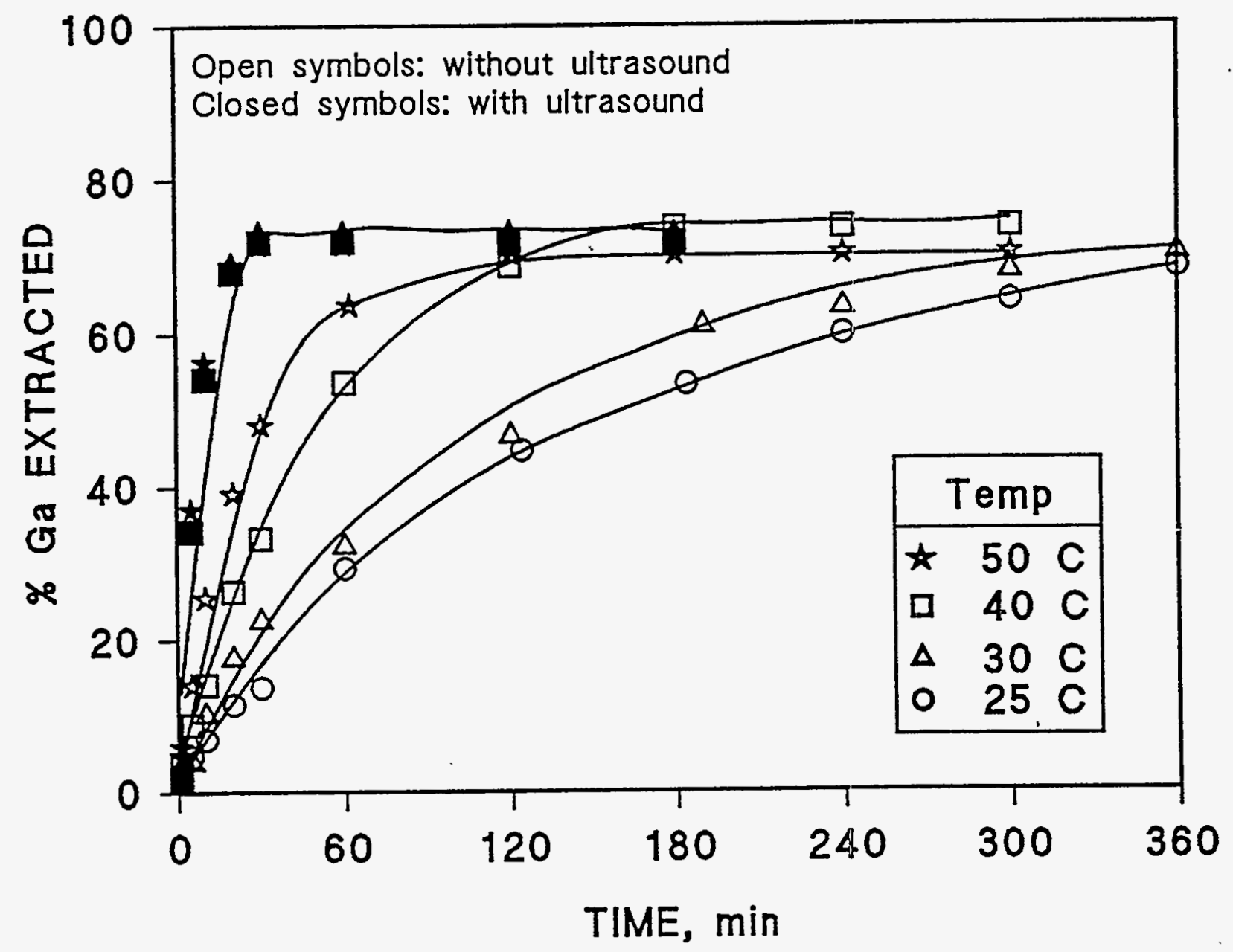

Figure 22. The effect of temperature on the solvent extraction of gallium with Kelex 100 decanol - Kermac 470B.

Organic phase, (v/v): $8 \%$ Kelex 100, 10\% decanol, $82 \%$ Kermac 470B.

Aqueous phase, $(\mathrm{g} / \mathrm{L}): 0.2 \mathrm{Ga}, 175 \mathrm{Na}_{2} \mathrm{O}, 80 \mathrm{Al}_{2} \mathrm{O}_{3}$.

System: O/A $=1: 1$, stirring speed $2000 \mathrm{rpm}$.

Ultrasound: $20 \mathrm{kHz}, 47 \mathrm{~W}$. 
Table 9. Apparent forward reaction rate constant, $\mathrm{k}_{\mathrm{f}}$ for the solvent extraction of gallium in the presence and in the absence of ultrasound (data from Figure 22).

\begin{tabular}{ccc}
\hline & \multicolumn{2}{c}{$\begin{array}{c}\mathrm{k}_{\mathrm{f}} \times 10^{4} \\
\left(\mathrm{sec}^{-1}\right)\end{array}$} \\
\cline { 2 - 3 } $\begin{array}{c}\text { Temperature } \\
\left({ }^{\circ} \mathrm{C}\right)\end{array}$ & Without Ultrasound & With Ultrasound \\
\hline 25 & 1.04 & - \\
30 & 1.30 & 18.5 \\
40 & 2.86 & 18.5 \\
50 & 4.52 & 18.2 \\
\hline
\end{tabular}

In general, temperature has a negative effect on the cavitation phenomena produced by ultrasound. It is unlikely that the expected reaction rate increase with an increase in temperature was exactly matched by a decrease in cavitation intensity to produce the observed null effect of temperature in the presence of ultrasound. It is more likely that the ultrasonic contribution to the rate phenomena far exceeded the temperature contribution. From the results of Part II, it can be recalled that, for the solvent extraction of gallium, the removal of water molecules from the gallate ion water shell is a critical and rate controlling step. It appears that, in the presence of ultrasound, the shock waves produced by cavitation are of sufficient intensity to disrupt the water shell, thus reducing the importance of this reaction mechanism on the overall rate of extraction.

\section{Effect of Diluents and Modifiers}

The sonochemical extraction of gallium was studied with four different diluents, Kermac 470B, Escaid 200, Aromatic 150, and cyclohexane, and the results are presented in Figure 23. For comparison purposes, the extraction data obtained with the same diluents in the absence of ultrasound are presented in Figure 24. The calculated extraction rates for the data presented in these two figures are given in Table 10.

The results clearly demonstrate that the presence of ultrasound was beneficial for all four systems, but it was the most beneficial for the system with cyclohexane, and the least beneficial with Aromatic 150. The reasons for the differences among the various diluent systems are difficult to explain, except when cyclohexane was used. In this case, a greatly increased interfacial surface area was responsible for the enhanced extraction rates, because very strong, paste-like emulsions were produced.

A major question to consider is the reason for the smaller increase in rate with the use of ultrasound when Aromatic 150 was used as the diluent, as compared to the other two diluents, Kermac 470B and Escaid 200. Although the exact answer is not known, it is believed that the solvation phenomena in the Aromatic 150 system is much stronger than in the others. The strong solvation properties of Aromatic 150 would keep the Kelex 100 molecule within the droplet and away from the interface, thus causing a slower extraction rate. 


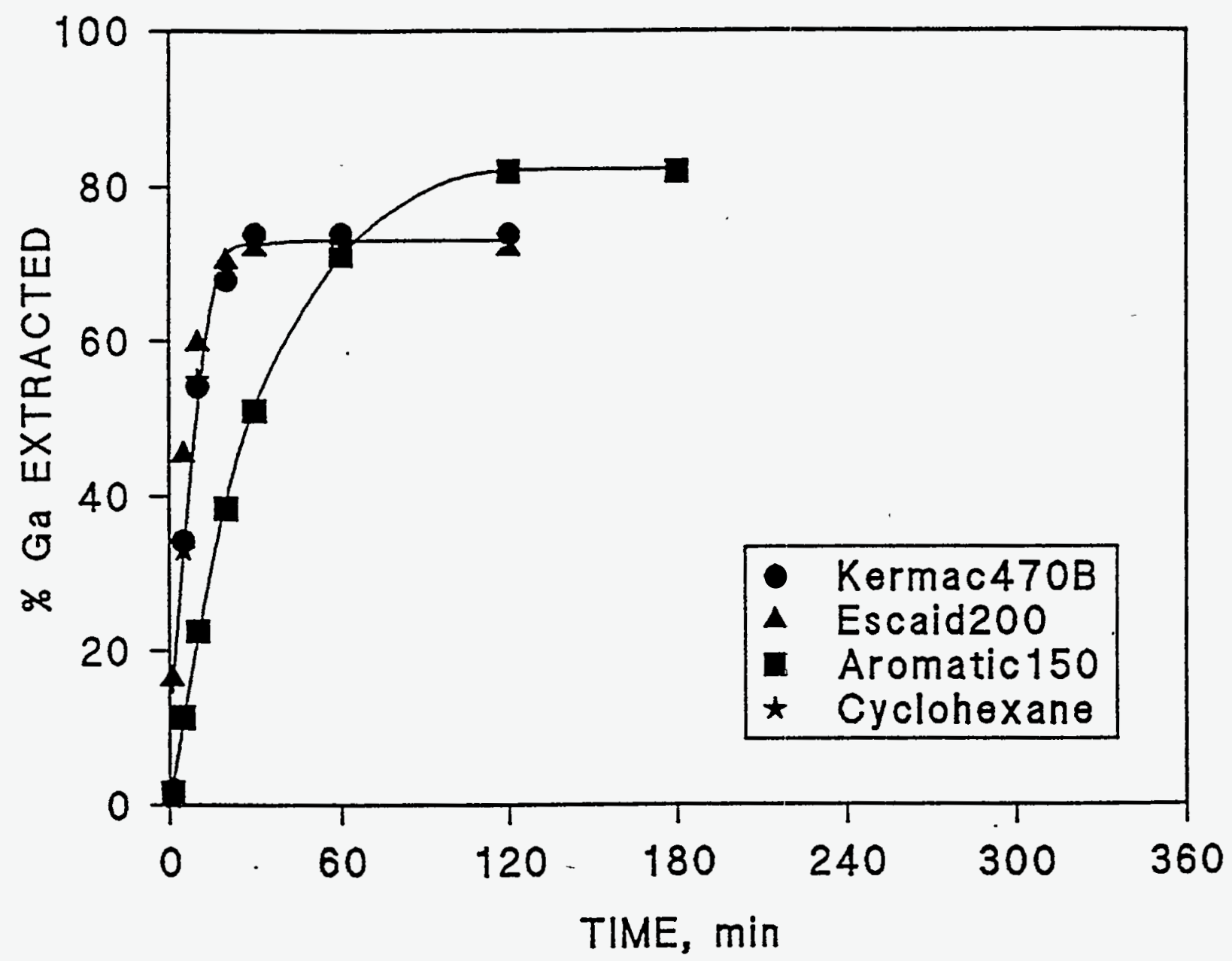

Figure 23. The extraction of gallium in the presence of ultrasound using four different diluents and decanol as a modifier.

Organic phase, (v/v): $8 \%$ Kelex 100, $10 \%$ decanol, $82 \%$ diluents.

Aqueous phase, $(\mathrm{g} / \mathrm{L}): 0.2 \mathrm{Ga}, 175 \mathrm{Na}_{2} \mathrm{O}, 80 \mathrm{Al}_{2} \mathrm{O}_{3}$.

System: $\mathrm{O} / \mathrm{A}=1: 1, T=40^{\circ} \mathrm{C}$, stirring speed $2000 \mathrm{rpm}$.

Ultrasound: $20 \mathrm{kHz}, 47 \mathrm{~W}$. 


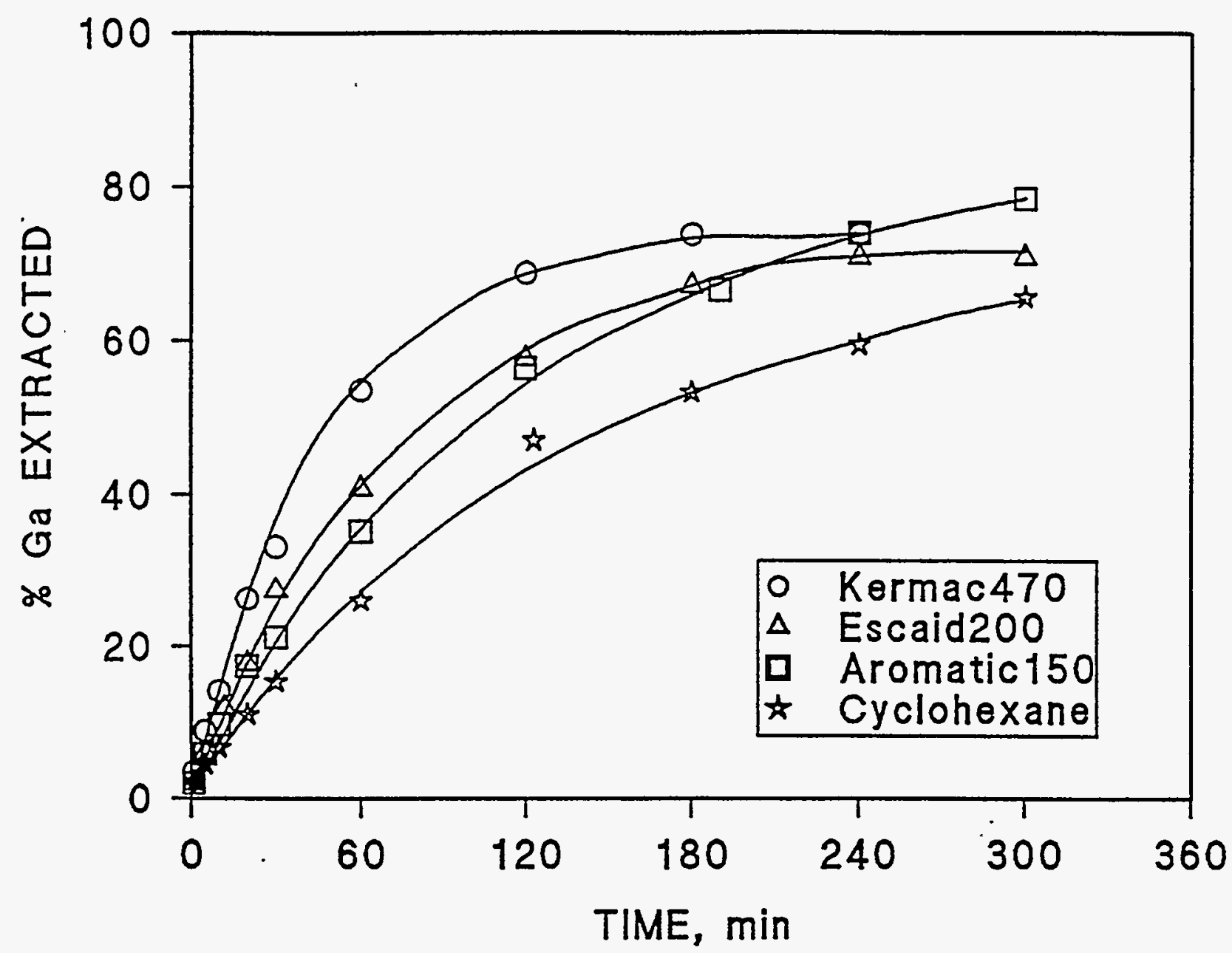

Figure 24. The extraction of gallium in the absence of ultrasound using four different diluents and decanol as a modifier..

Organic phase, $(\mathrm{v} / \mathrm{v}): 8 \%$ Kelex 100, $10 \%$ decanol, $82 \%$ diluents.

Aqueous phase, $(\mathrm{g} / \mathrm{L})$ : $0.2 \mathrm{Ga}, 175 \mathrm{Na}_{2} \mathrm{O}, 80 \mathrm{Al}_{2} \mathrm{O}_{3}$.

System: $\mathrm{O} / \mathrm{A}=1: 1, T=40^{\circ} \mathrm{C}$, stirring speed $2000 \mathrm{rpm}$. 
Table 10. The effect of diluents on the apparent forward reaction rate constant, $k_{f}$ for the solvent extraction of gallium in the presence and the absence of ultrasound using decanol as a modifier (data from Figures 23 and 24).

\begin{tabular}{lcccc}
\hline & & \multicolumn{2}{c}{$\mathrm{k}_{\mathrm{f}} \times 10^{4}$} \\
& & \multicolumn{2}{c}{$\left(\mathrm{sec}^{-1}\right)$} \\
\cline { 3 - 4 } \multicolumn{1}{c}{ Diluent } & Diluent Boiling & Without & With & \% Rate \\
Range $\left({ }^{\circ} \mathrm{C}\right)$ & Ultrasound & Ultrasound & Increase \\
\hline Kelex 100 - Decanol - Kermac 470B & $204-260$ & 2.86 & 18.47 & 645 \\
Kelex 100 - Decanol - Aromatic 150 & $176-210$ & 1.62 & 4.82 & 297 \\
Kelex 100 - Decanol - Escaid 200 & $185-221$ & 1.95 & 22.15 & 1136 \\
Kelex 100 - Decanol - Cyclohexane & 80.7 & 1.24 & 19.71 & 1589 \\
\hline
\end{tabular}

In the study of the effect of modifiers on the rate of solvent extraction of gallium without ultrasound (Part $\Pi$ of this report) it was found that ketones performed far better than alcohols. For that reason, both the effect of diluents and the effect of modifiers on the sonochemical extraction of gallium were examined. Decanol (as representative of alcohols) was used as a modifier for the data presented in Figures 23, 24, and Table 10. The same systems were then investigated using 2-undecanone (representing ketones) as a modifier, and the results are presented in Figures 25 and 26, and in Table 11.

It is important to note that ultrasound had no influence on the extraction rates with Kermac 470B and Escaid 200 diluents when 2-undecanone was used as a modifier. In these systems extraction occurs very rapidly in the absence of ultrasound, and with these fast systems, ultrasound cannot provide much improvement.

\section{Effect of Sodium Hydroxide and Aluminum Hydroxide Concentration}

The sonochemical extraction of gallium with respect to sodium hydroxide and aluminum hydroxide concentrations was examined by using the Kelex 100 - decanol - Kermac 470B system.

In the absence of ultrasound, sodium hydroxide has a very complex effect on the extraction of gallium, as shown in this work (Part II of this report) and the work of Sato and Oishi. ${ }^{65}$ An increase in sodium hydroxide concentration causes an increase in extraction rates, but only up to a certain concentration limit, beyond which a further increase is detrimental. With respect to the uptake of gallium, an increase in sodium hydroxide concentration decreases the amount of gallium transferred into the organic phase. The negative effect of sodium hydroxide can be explained by the competitive extraction of sodium and gallium.

As shown in Figure 27 and Table 12, the use of ultrasound causes a significant increase in the extraction rates as compared to the data obtained without the use of ultrasound (Figure 16 presented in Part II). In the presence of ultrasound, the extraction equilibriums were reached within $20 \mathrm{~min}$. From Figure 27 it can be noted that increasing the sodium hydroxide concentration has a significantly detrimental effect on gallium uptake. 


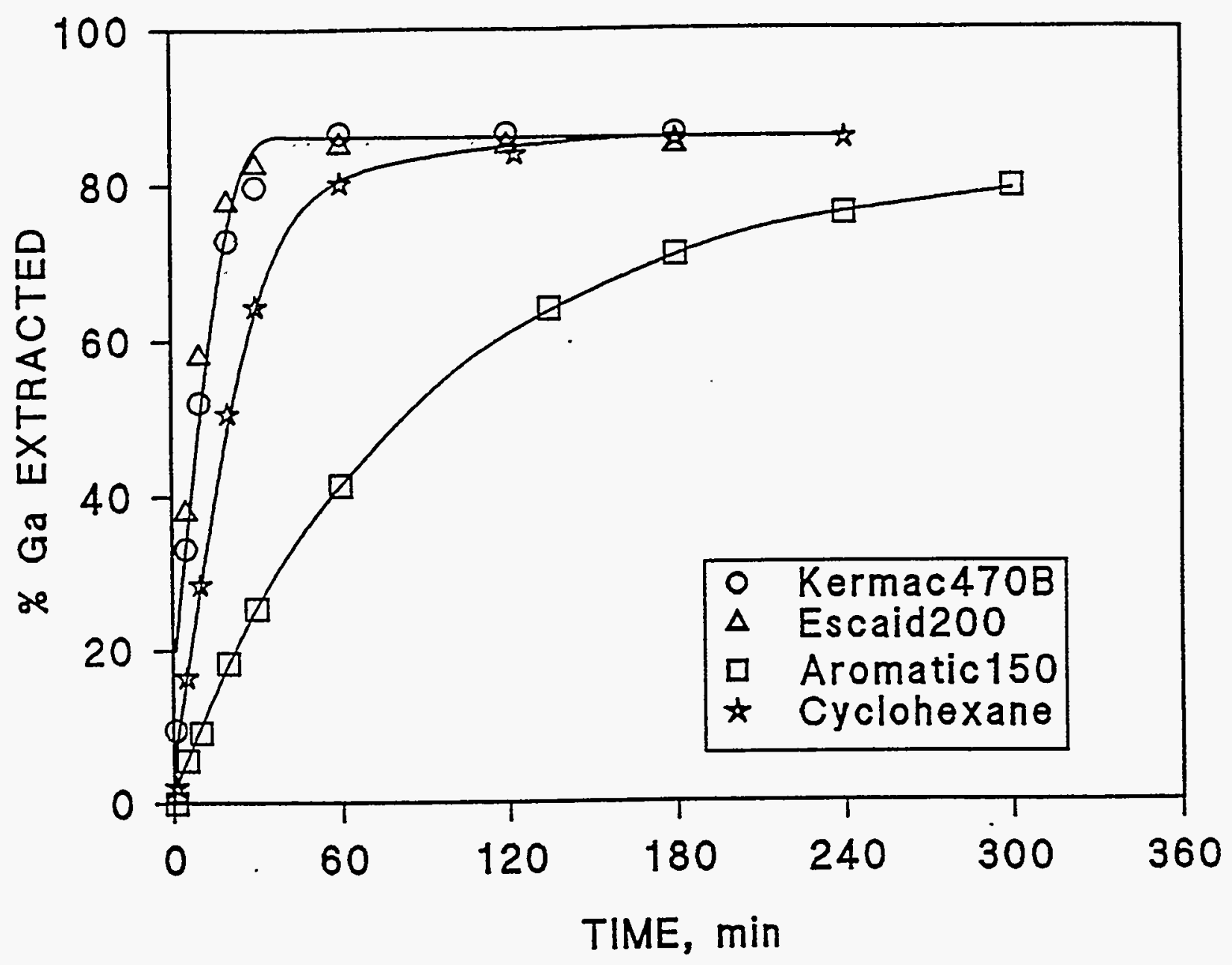

Figure 25. The extraction of gallium in the absence of ultrasound using four different diluents and 2-undecanone as a modifier.

Organic phase, $(\mathrm{v} / \mathrm{v}): 8 \%$ Kelex $100,10 \%$ 2-undecanone, $82 \%$ diluents.

Aqueous phase, $(\mathrm{g} / \mathrm{L}): 0.2 \mathrm{Ga}, 175 \mathrm{Na}_{2} \mathrm{O}, 80 \mathrm{Al}_{2} \mathrm{O}_{3}$.

System: $\mathrm{O} / \mathrm{A}=1: 1, T=40^{\circ} \mathrm{C}$, stirring speed $2000 \mathrm{rpm}$. 


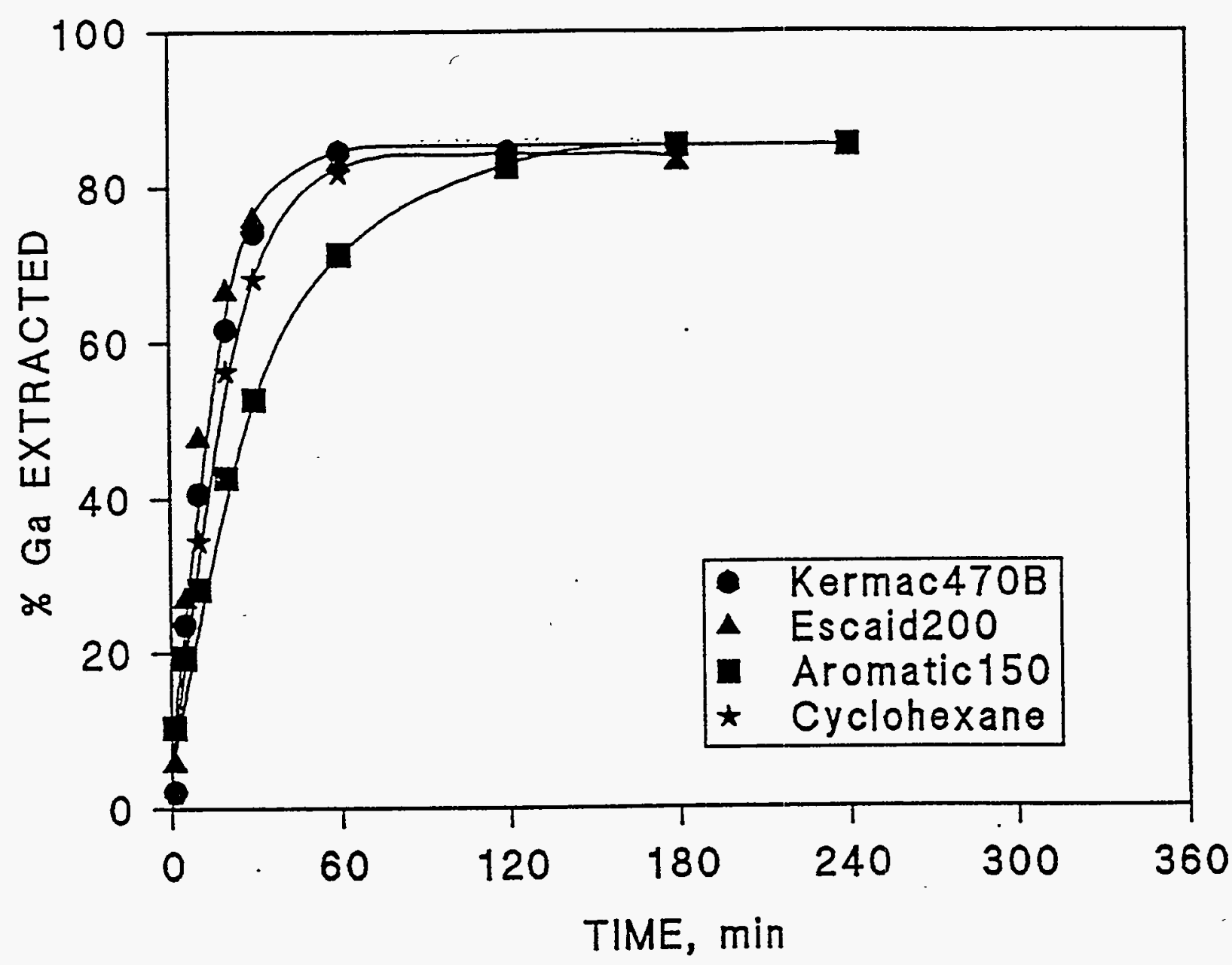

Figure 26. The extraction of gallium in the presence of ultrasound using four different diluents and 2-undecanone as a modifier.

Organic phase, (v/v): $8 \%$ Kelex 100, 10\% 2-undecanone, $82 \%$ diluents.

Aqueous phase, $(\mathrm{g} / \mathrm{L}): 0.2 \mathrm{Ga}, 175 \mathrm{Na}_{2} \mathrm{O}, 80 \mathrm{Al}_{2} \mathrm{O}_{3}$.

System: $\mathrm{O} / \mathrm{A}=1: 1, T=40^{\circ} \mathrm{C}$, stirring speed $2000 \mathrm{rpm}$.

Ultrasound: $20 \mathrm{kHz}, 47 \mathrm{~W}$. 
Table 11. The effect of diluents on the apparent forward reaction rate constant, $k_{\mathfrak{f}}$, for solvent extraction of gallium in the presence and in the absence of ultrasound using 2-undecanone as a modifier (data from Figures 25 and 26).

\begin{tabular}{|c|c|c|c|}
\hline \multirow[b]{2}{*}{ Diluent } & \multicolumn{2}{|c|}{$\begin{array}{c}\mathbf{k}_{\mathrm{f}} \times 10^{4} \\
\left(\mathrm{sec}^{-1}\right)\end{array}$} & \multirow[b]{2}{*}{$\begin{array}{l}\text { \% Rate } \\
\text { Increase }\end{array}$} \\
\hline & $\begin{array}{l}\text { Without } \\
\text { Ultrasound }\end{array}$ & $\begin{array}{c}\text { With } \\
\text { Ultrasound }\end{array}$ & \\
\hline Kelex 100 - 2-undecanone - Kermac 470B & 12.47 & 12.00 & none \\
\hline Kelex 100 - 2-undecanone - Aromatic 150 & 1.79 & 4.07 & 227 \\
\hline Kelex 100 - 2-undecanone - Escaid 200 & 17.32 & 16.97 & none \\
\hline
\end{tabular}

Table 12. The effect of sodium hydroxide concentration on the extraction rates of gallium in the absence and in the presence of ultrasound (data taken from Figure 16 and Figure 27).

\begin{tabular}{ccc}
\hline & \multicolumn{2}{c}{$\begin{array}{c}\mathrm{k}_{\mathrm{f}} \times 10^{4} \\
\left(\mathrm{sec}^{-1}\right)\end{array}$} \\
\cline { 2 - 3 } $\mathrm{Na}_{2} \mathrm{O}$ Concentration \\
$(\mathrm{g} / \mathrm{L})$ & Without Ultrasound & With Ultrasound \\
\hline 102 & 1.80 & 19.5 \\
138 & 2.23 & 37.0 \\
175 & 2.86 & 33.3 \\
210 & 2.03 & 21.3 \\
\hline
\end{tabular}

With regard to the effect of sodium hydroxide on the kinetics of extraction, the results obtained in the presence of ultrasound are similar to those obtained in the absence of ultrasound. As shown in Table 12, an initial increase of sodium hydroxide concentration results in an increase in extraction rate, but beyond a certain concentration limit the extraction rate decreases.

The results of tests with solutions having varying concentrations of aluminum hydroxide are presented in Figures 28 and 29, and in Table 13. In the absence of ultrasound (Figure 28) the presence of aluminum hydroxide is detrimental for the extraction rate and uptake of gallium. Because aluminum extraction with Kelex 100 is extremely slow, the competitive extraction of aluminum cannot be the cause of its negative effect on extraction of gallium.

In the presence of ultrasound, aluminum hydroxide also had a negative effect on the extraction of gallium (Figure 29). Increased concentrations of aluminum hydroxide results in a reduced rate and total uptake of gallium. The effect of aluminum hydroxide on the extraction rates of gallium in the absence and presence of ultrasound is quantitatively presented in Table 13. The negative effect of aluminum hydroxide can be explained as being the result of the negative influence of aluminum on the dehydration of gallate ions, as presented in Part II of this report. 


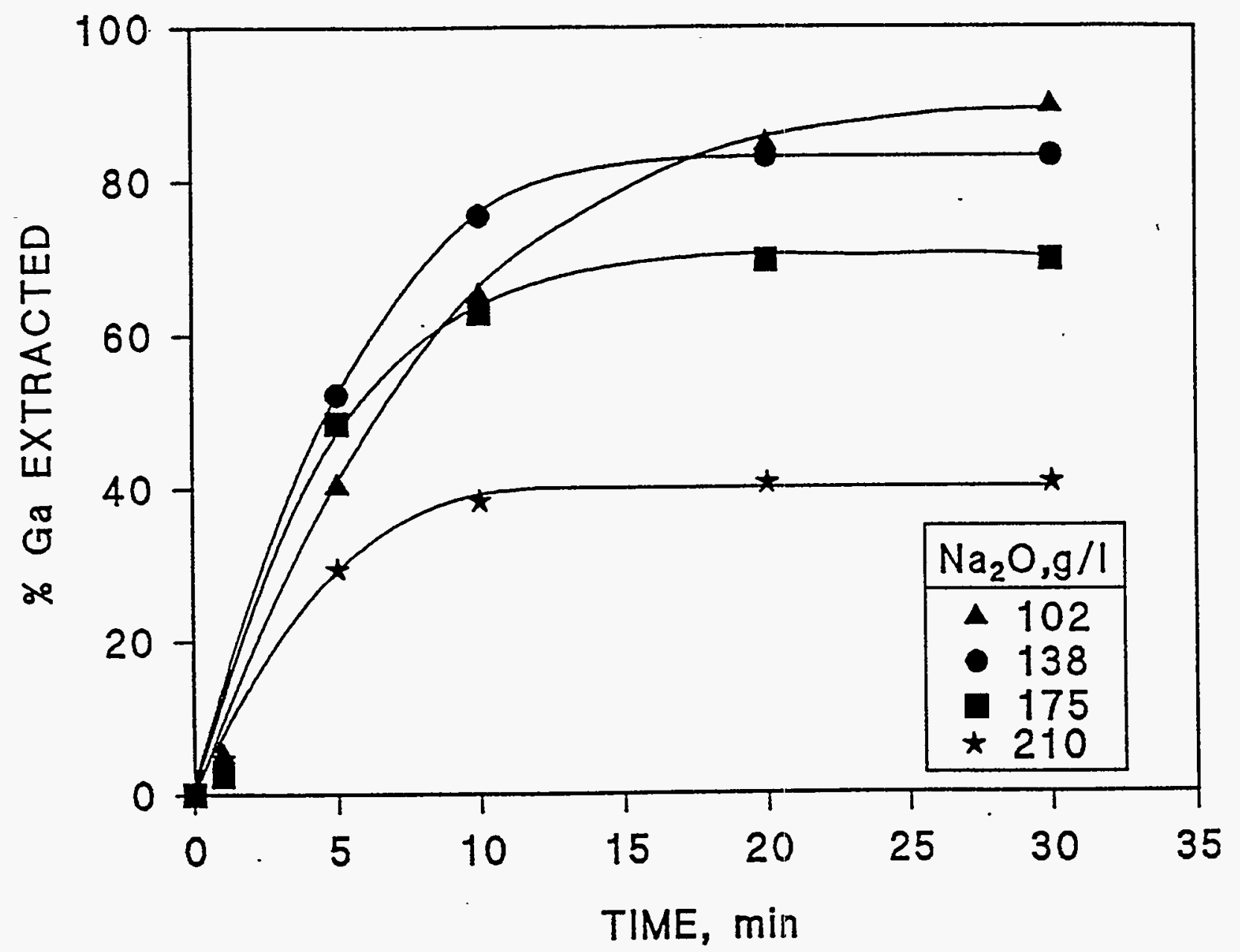

Figure 27. The effect of sodium hydroxide concentration on the extraction of gallium in the presence of ultrasound.

Organic phase, (v/v): $8 \%$ Kelex 100, 10\% decanol, 82\% Kermac 470B.

Aqueous phase, $(\mathrm{g} / \mathrm{L}): 0.2 \mathrm{Ga}$, indicated $\mathrm{Na}_{2} \mathrm{O}, 80 \mathrm{Al}_{2} \mathrm{O}_{3}$.

System: $\mathrm{O} / \mathrm{A}=1: 1$, stirring speed $2000 \mathrm{rpm}$.

Ultrasound: $20 \mathrm{kHz}, 47 \mathrm{~W}$. 


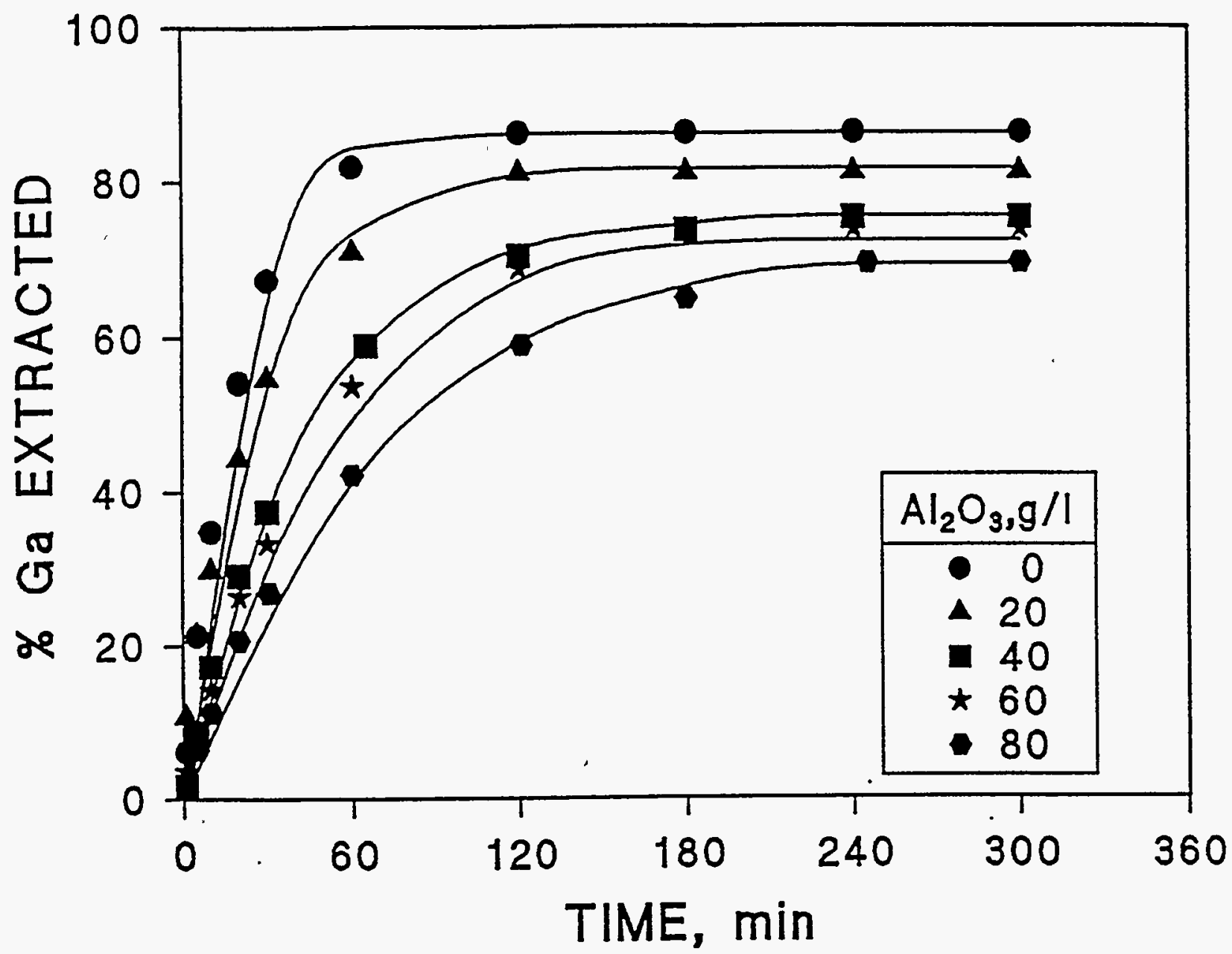

Figure 28. The effect of alumina concentration on the solvent extraction of gallium in the absence of ultrasound.

Organic phase, (v/v): $8 \%$ Kelex 100, 10\% decanol, $82 \%$ Kermac 470B.

Aqueous phase, $(\mathrm{g} / \mathrm{L}): 0.2 \mathrm{Ga}, 175 \mathrm{Na}_{2} \mathrm{O}$, indicated $\mathrm{Al}_{2} \mathrm{O}_{3}$.

System: $\mathrm{O} / \mathrm{A}=1: 1$, stirring speed $2000 \mathrm{rpm}$. 


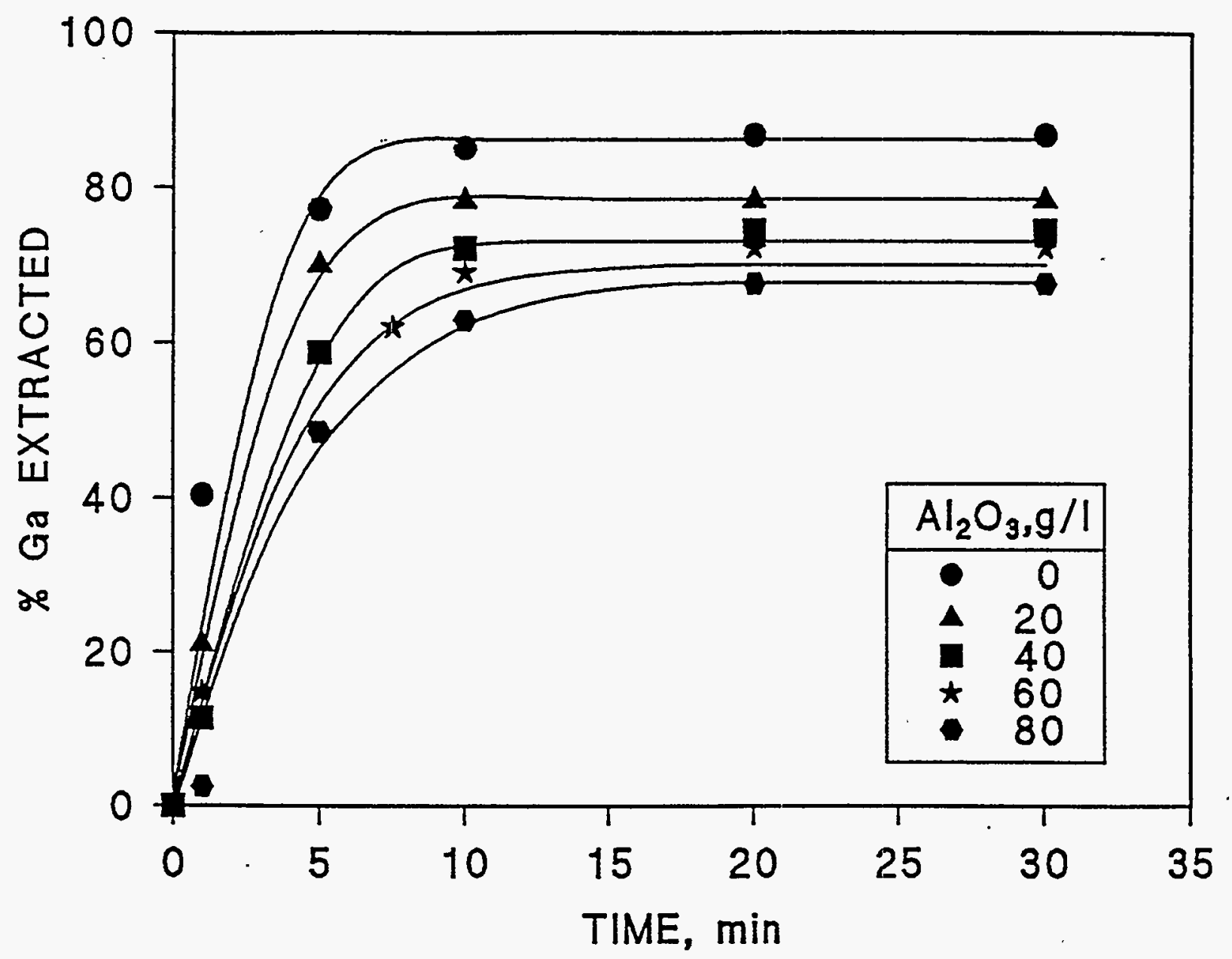

Figure 29. The effect of alumina concentration on the solvent extraction of gallium in the presence of ultrasound.

Organic phase, $(\mathrm{v} / \mathrm{v}): 8 \%$ Kelex 100, $10 \%$ decanol, $82 \%$ Kermac $470 \mathrm{~B}$.

Aqueous phase, $(\mathrm{g} / \mathrm{L}): 0.2 \mathrm{Ga}, 175 \mathrm{Na}_{2} \mathrm{O}$, indicated $\mathrm{Al}_{2} \mathrm{O}_{3}$.

System: $\mathrm{O} / \mathrm{A}=1: 1$, stirring speed $2000 \mathrm{rpm}$.

Ultrasound: $20 \mathrm{kHz}, 47 \mathrm{~W}$. 
Table 13. The effect of aluminum hydroxide concentration on the extraction rates of gallium in the absence and in the presence of ultrasound (data taken from Figures 28 and 29).

\begin{tabular}{ccc}
\hline & \multicolumn{3}{c}{$\begin{array}{c}\mathrm{k}_{\mathrm{f}} \times 10^{4} \\
\left(\mathrm{sec}^{-1}\right)\end{array}$} \\
\cline { 2 - 3 } $\mathrm{Al}_{2} \mathrm{O}_{3}$ Concentration \\
$(\mathrm{g} / \mathrm{L})$ & Without Ultrasound & With Ultrasound \\
\hline 0 & 7.29 & 54.9 \\
20 & 4.18 & 47.8 \\
40 & 2.83 & 42.0 \\
60 & 2.88 & 36.8 \\
80 & 2.86 & 33.3 \\
\hline
\end{tabular}

\section{Solvent Extraction Isotherm}

The equilibrium isotherm for gallium extraction with the Kelex 100 - decanol - Kermac 470B system is presented in Figure 30 . At $40^{\circ} \mathrm{C}$, the maximum loading of Kelex 100 with gallium is about $3.1 \mathrm{~g} / \mathrm{L}$ of organic phase. The effect of ultrasound on the loading isotherm is also presented in this figure. It can be seen that ultrasound did not change the equilibrium loading characteristics of Kelex 100.

\section{Sonochemical Extraction of Gallium from an Industrial Process Solution}

The effect of ultrasound on the solvent extraction of gallium from an industrial process solution was studied using Bayer leach liquor provided by Kaiser Aluminum Co. The composition of the liquor solution is given in Table 14.

The solvent extraction of gallium was studied as a function of diluents and modifiers. In the solvent extraction system, the organic phase consisted of $8 \%(\mathrm{v} / \mathrm{v})$ Kelex 100 extractant, $10 \%(\mathrm{v} / \mathrm{v})$ modifier, and $82 \%(\mathrm{v} / \mathrm{v})$ diluent. The O/A ratio was $1: 1$, and the total volume in the reactor was $200 \mathrm{~mL}$. The standard temperature was $40^{\circ} \mathrm{C}$, and the ultrasonic power input was $28 \mathrm{~W}$.

\section{Effect of Diluents}

The solvent extraction of gallium, in the absence and presence of ultrasound, was studied with three diluents, Kermac 470B, Escaid 200, and Aromatic 150. Decanol was used as the modifier in each case. The results are presented in Figures 31 and 32, and in Table 15. As can be seen from the results, the solvent extraction of gallium from real solutions is very slow, and it took longer than six hours to achieve equilibrium. The extraction rate is much slower than that observed for the synthetic sodium aluminate solutions. However, similar to the solvent extraction from the synthetic solutions, the rate of extraction was the slowest when Aromatic 150 was used as the diluent. The rates were dramatically enhanced when ultrasound was used, and the rates were the most enhanced with Escaid 200 as the diluent. 


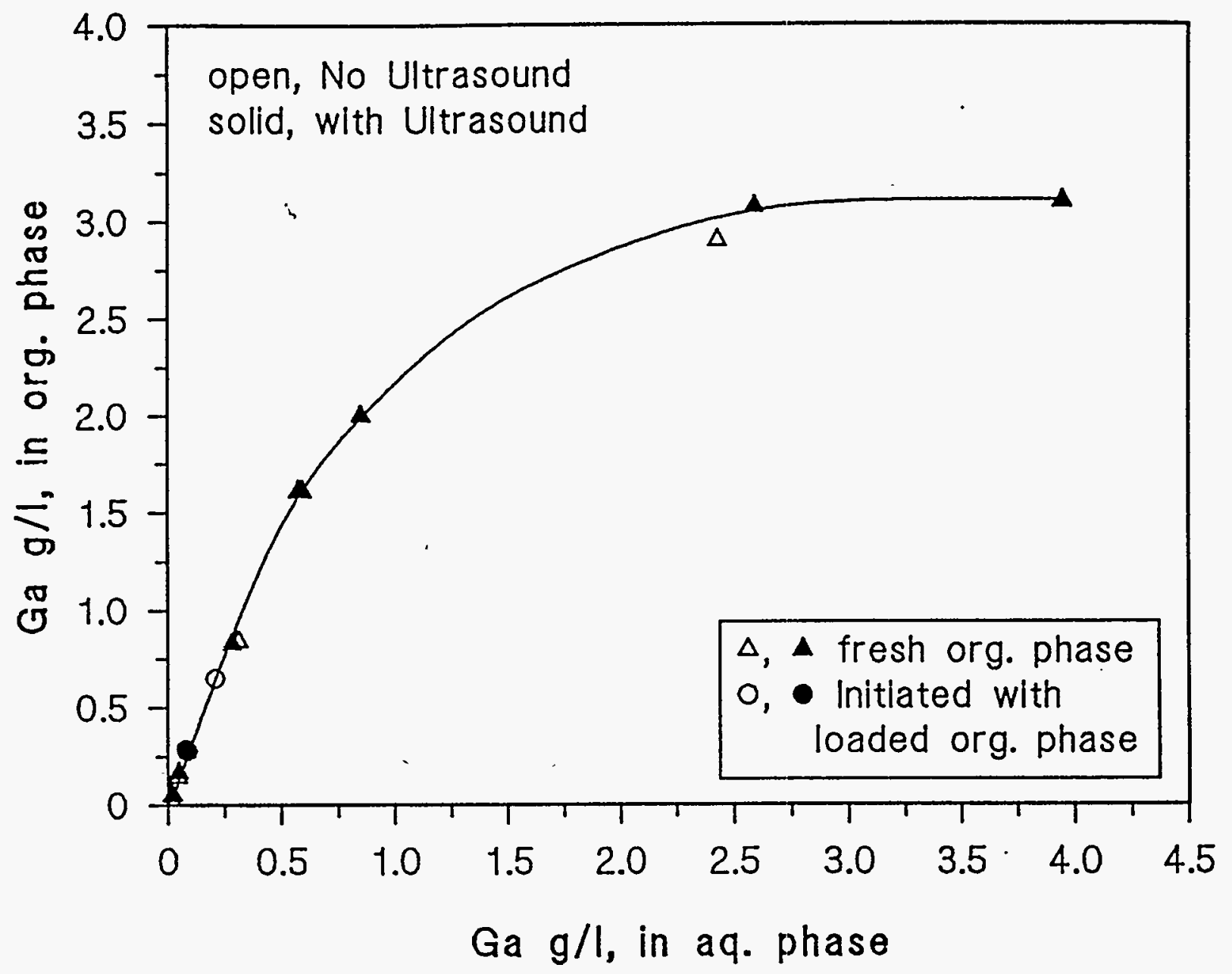

Figure 30. Extraction isotherm of gallium with Kelex 100 - decanol - Kermac 470B.

Organic phase, (v/v): $8 \%$ Kelex 100, 10\% decanol, 82\% Kermac 470B.

Aqueous phase, $(\mathrm{g} / \mathrm{L})$ : Indicated $\mathrm{Ga}, 175 \mathrm{Na}_{2} \mathrm{O}, 80 \mathrm{Al}_{2} \mathrm{O}_{3}$.

System: $T=40^{\circ} \mathrm{C}$, stirring speed $2000 \mathrm{rpm}$.

Ultrasound: $20 \mathrm{kHz}, 32.5 \mathrm{~W}$. 
Table 14. The composition of the industrial Bayer process leach liquor (evaporator discharge) from Kaiser Aluminum Company.

\begin{tabular}{lccc}
\hline \multicolumn{1}{c}{ Chemical } & $\begin{array}{c}\text { Concentration } \\
(\mathrm{g} / \mathrm{L})\end{array}$ & Chemical & $\begin{array}{c}\text { Concentration } \\
(\mathrm{g} / \mathrm{L})\end{array}$ \\
\hline $\mathrm{Na}_{2} \mathrm{CO}_{3}$ & 252.0 & $\mathrm{Al}_{2} \mathrm{O}_{3}$ & 111.3 \\
Organic Soda & 24.1 & $\mathrm{NaCl}$ & 17.6 \\
Total Soda & 305.1 & $\mathrm{Na}_{2} \mathrm{SO}_{4}$ & 61.1 \\
Organic Carbon & 12.18 & $\mathrm{Na}_{2} \mathrm{C}_{2} \mathrm{O}_{4}$ & 2.4 \\
$\mathrm{SiO}_{2}$ & 0.608 & $\mathrm{Ga}$ & 0.125 \\
\hline
\end{tabular}

Table 15. The effect of diluents on the apparent forward reaction rate constant, $k_{f}$ for the solvent extraction of gallium from an industrial process solution in the presence and in the absence of ultrasound (data from Figures 31 and 32).

\begin{tabular}{lccc}
\hline & \multicolumn{2}{c}{$\begin{array}{c}\mathrm{k}_{\mathrm{f}} \times 10^{4} \\
\left(\mathrm{sec}^{-1}\right)\end{array}$} \\
\cline { 2 - 4 } \multicolumn{1}{c}{ Diluent } & $\begin{array}{c}\text { Without } \\
\text { Ultrasound }\end{array}$ & $\begin{array}{c}\text { With } \\
\text { Ultrasound }\end{array}$ & \% Rate Increase \\
\hline Kelex 100 - Decanol - Kermac 470B & 1.00 & 5.50 & 550 \\
Kelex 100 - Decanol - Aromatic 150 & 0.64 & 2.60 & 406 \\
Kelex 100 - Decanol - Escaid 200 & 0.73 & 8.00 & 1095 \\
\hline
\end{tabular}

\section{Modifier Effect}

The performance of two modifiers, decanol and 2-undecanone, was evaluated using three diluents, Kermac 470B, Aromatic 150, and Escaid 200. The extractions of gallium were performed in the presence of ultrasound. The results of these experiments are given in Figures 33 through 35, and in Table 16.

With each diluent, 2-undecanone produced higher extraction rates than decanol. The fastest extraction rates were obtained when Escaid 200 was used as a diluent, and the slowest rates were obtained with Aromatic 150. However, systems with Aromatic 150 responded with higher uptake of gallium. For each diluent, 2-undecanone also produced higher uptake rates of gallium than decanol. 


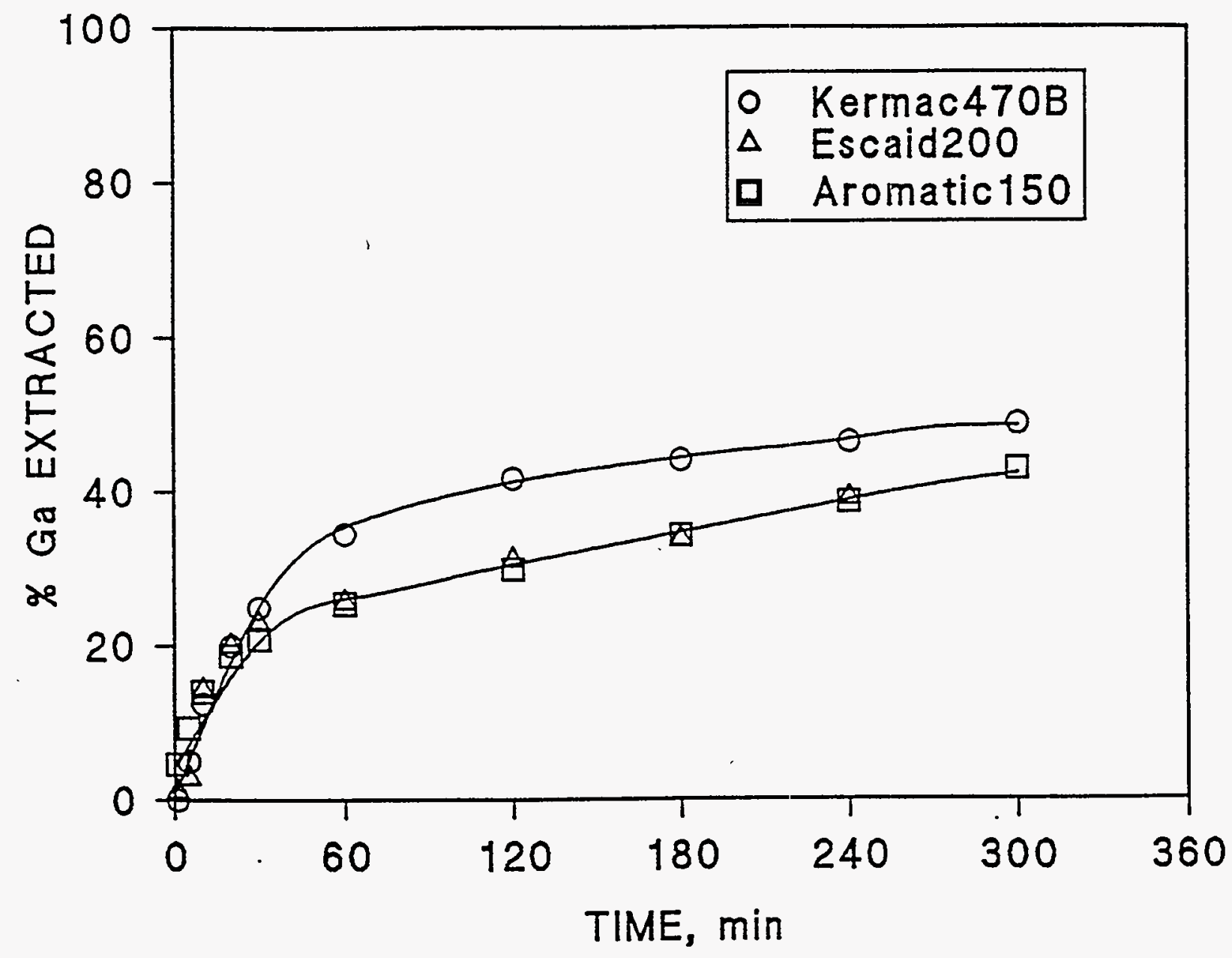

Figure 31. The effect of diluents on the extraction of gallium from an industrial process solution in the absence of ultrasound.

Organic phase, (v/v): $8 \%$ Kelex 100, 10\% decanol, $82 \%$ diluent.

Aqueous phase: See Table 14.

System: $\mathrm{O} / \mathrm{A}=1: 1$, stirring speed $2000 \mathrm{rpm}$. 


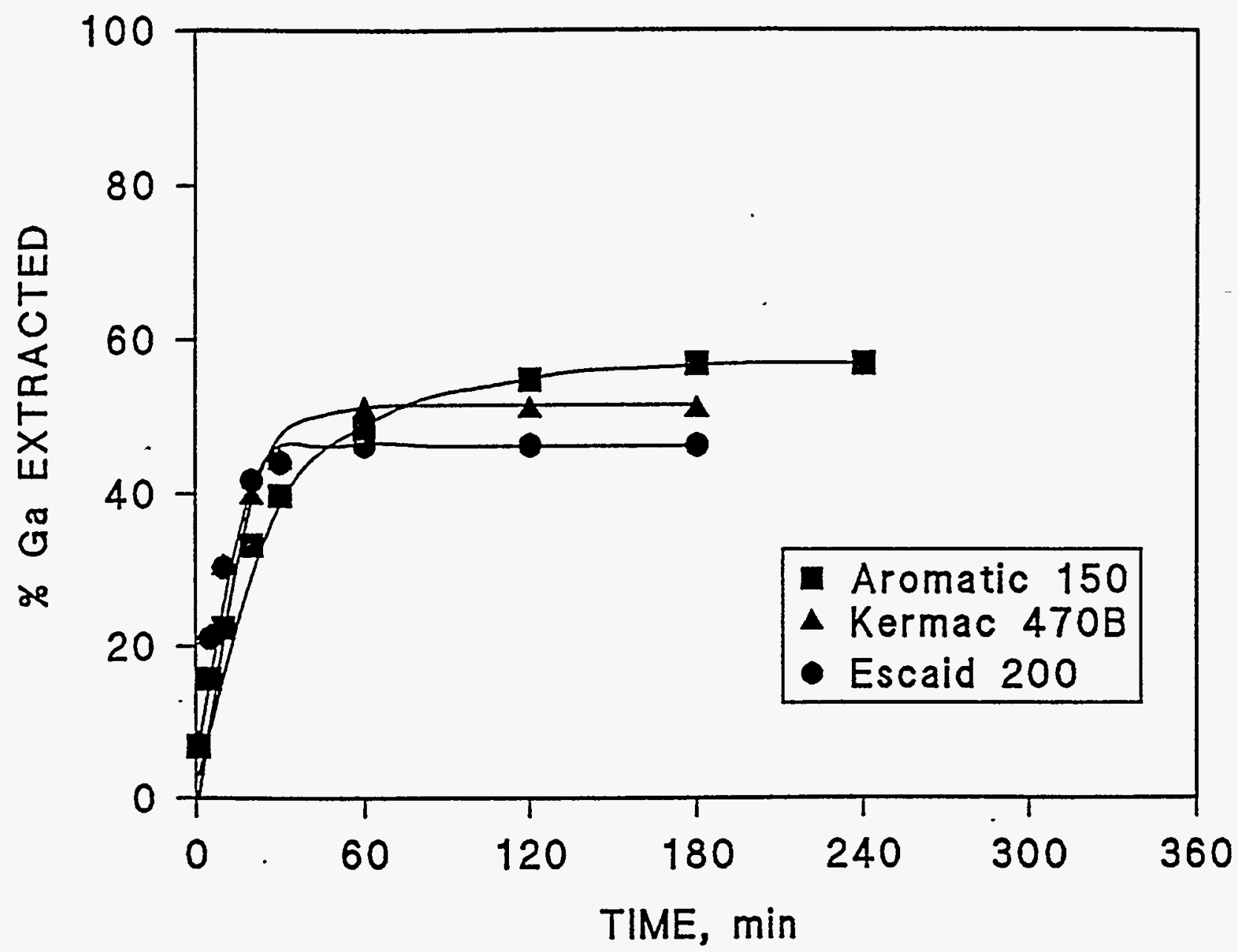

Figure 32. The effect of diluents on the extraction of gallium from an industrial process solution in the presence of ultrasound.

Organic phase, (v/v): $8 \%$ Kelex $100,10 \%$ decanol, $82 \%$ diluent.

Aqueous phase: See Table 14.

System: $\mathrm{O} / \mathrm{A}=1: 1$, stirring speed $2000 \mathrm{rpm}$.

Ultrasound: $20 \mathrm{kHz}, 28 \mathrm{~W}$. 


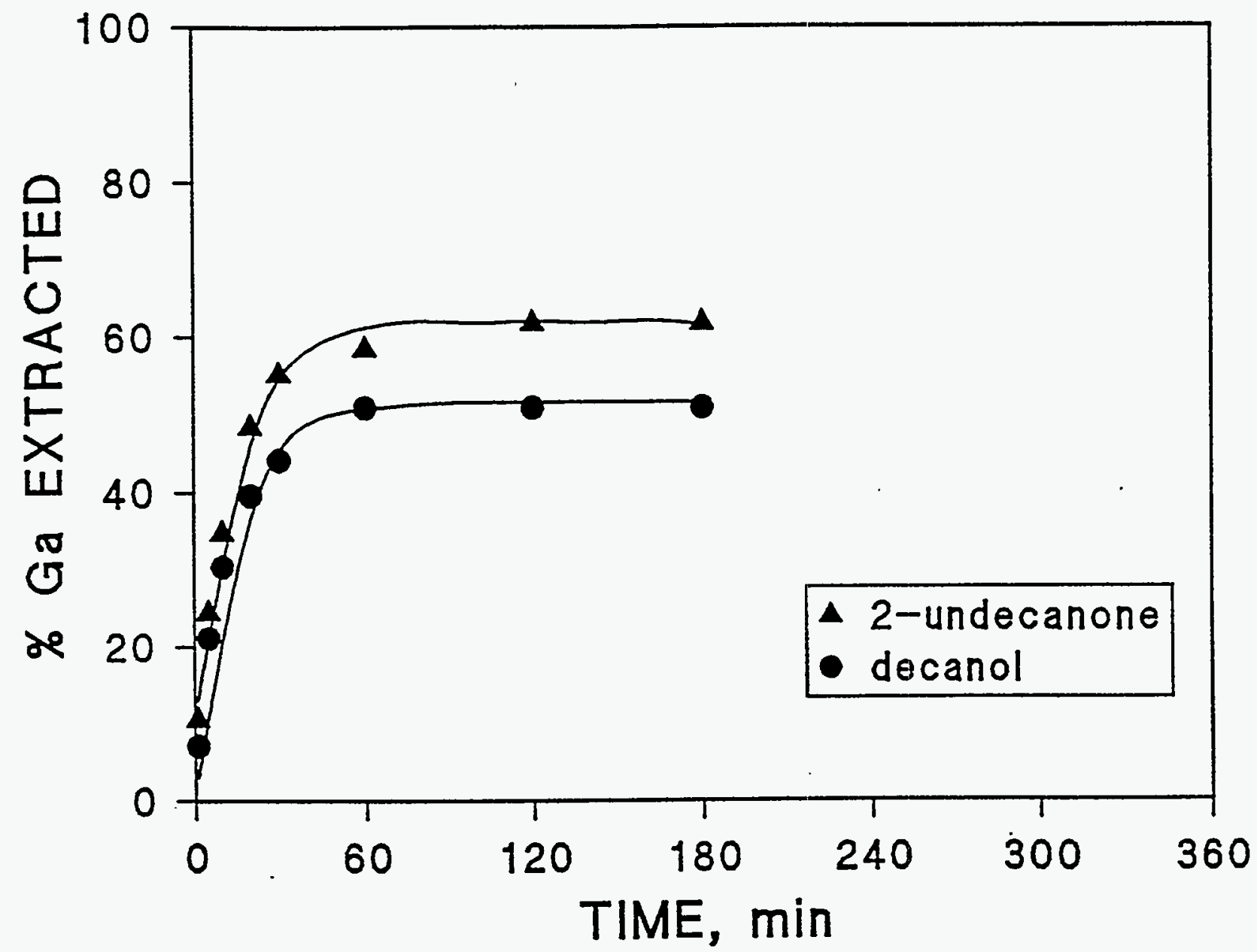

Figure 33. The effect of modifiers with Kermac 470B on the extraction of gallium from an industrial process solution in the presence of ultrasound.

Organic phase, (v/v): $8 \%$ Kelex $100,10 \%$ modifier, $82 \%$ Kermac $470 \mathrm{~B}$.

Aqueous phase: See Table 14.

System: $\mathrm{O} / \mathrm{A}=1: 1$, stirring speed $2000 \mathrm{rpm}$.

Ultrasound: $20 \mathrm{kHz}, 28 \mathrm{~W}$. 


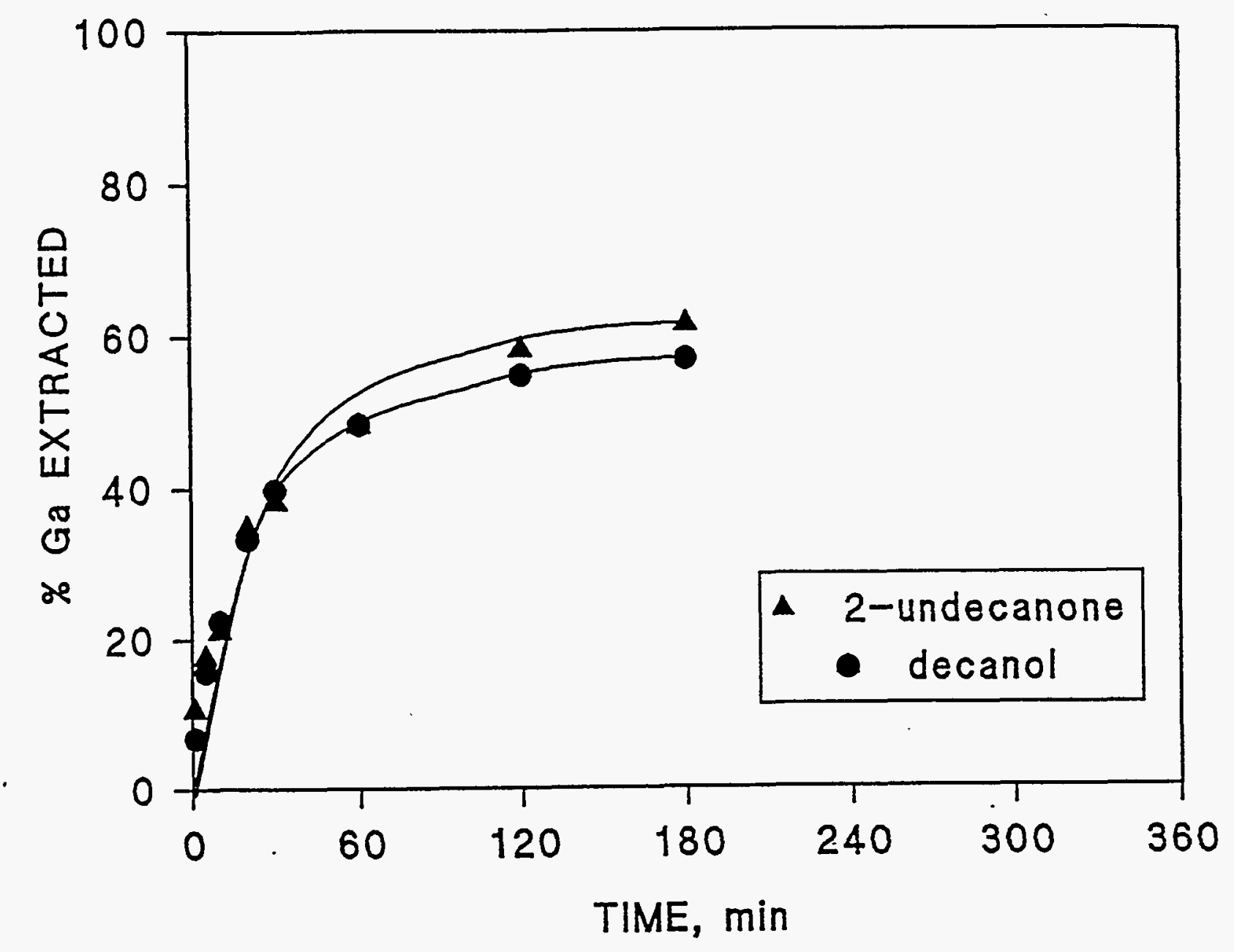

Figure 34. The effect of modifiers with Aromatic 150 on the extraction of gallium from an industrial process solution in the presence of ultrasound.

Organic phase, (v/v): $8 \%$ Kelex 100, 10\% modifier, 82\% Aromatic 150.

Aqueous phase: See Table 14.

System: $\mathrm{O} / \mathrm{A}=1: 1$, stirring speed $2000 \mathrm{rpm}$.

Ultrasound: $20 \mathrm{kHz}, 28 \mathrm{~W}$. 


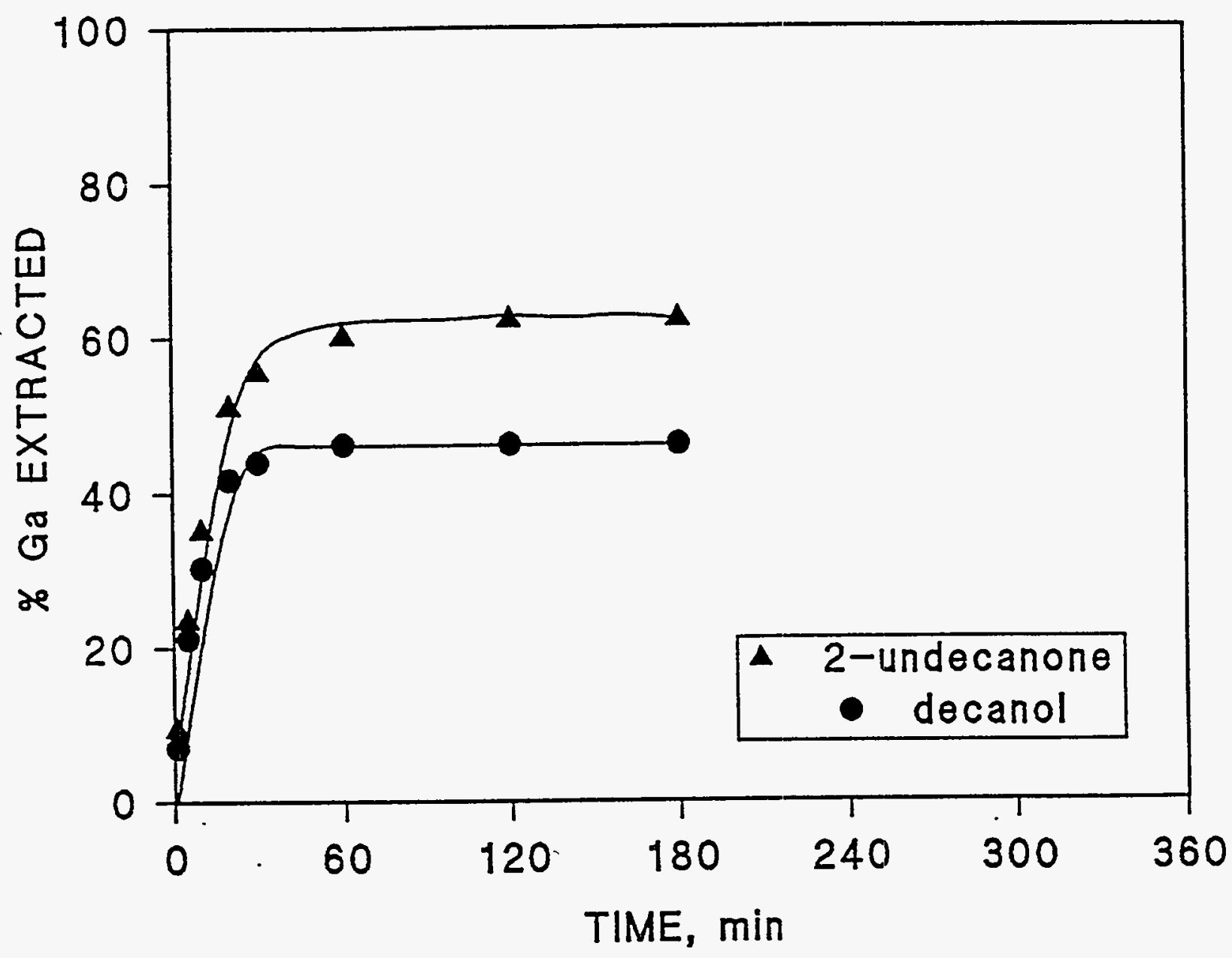

Figure 35. The effect of modifiers with Escaid 200 on the extraction of gallium from an industrial process solution in the presence of ultrasound.

Organic phase, (v/v): 8\% Kelex 100, 10\% modifier, 82\% Escaid 200.

Aqueous phase: See Table 14.

System: $\mathrm{O} / \mathrm{A}=1: 1$, stirring speed $2000 \mathrm{rpm}$.

Ultrasound: $20 \mathrm{kHz}, 28 \mathrm{~W}$. 
Table 16. The effect of modifiers on the rate of extraction of gallium from Bayer process leach liquor in the presence of ultrasound.

\begin{tabular}{lccc}
\hline & \multicolumn{3}{c}{$\begin{array}{c}\mathrm{k}_{\mathrm{f}} \times 10^{4} \\
\left(\mathrm{sec}^{-1}\right)\end{array}$} \\
\cline { 2 - 4 } Modifier & Kermac 470B & Aromatic 150 & Escaid 200 \\
\hline Decanol & 5.5 & 2.6 & 8.0 \\
2-undecanone & 7.3 & 2.5 & 8.6 \\
\hline
\end{tabular}

\section{Stability of Kelex 100}

The high price of Kelex 100 demands, in addition to its complete recycling, applications in which it will maintain its chemical stability. Because ultrasonic cavitation effects could possibly rupture the chemical bonds in Kelex 100, it was considered important to examine the stability of the extractant in the presence of ultrasound. A separate sample of Kelex 100 was subjected to a higher intensity ultrasonic treatment ( $47 \mathrm{~W}$ input), continuously for six hours. The IR and the NMR spectra before and after the ultrasonic treatment were identical, indicating a very high stability for this extractant.

\section{Energy Consumption}

Although the benefits of ultrasonic presence during solvent extraction of gallium are many, ultrasonic treatment requires a large amount of energy. In a batch operation, the continuous presence of ultrasound would consume a large amount of energy depending on the power input and duration of treatment. One approach to reduce the energy consumption would be to use the ultrasound intermittently rather than continuously.

Similar to the case studied with nickel extraction, it was found that the solvent extraction of gallium does not require a continuous application of ultrasound. An additional study was performed using an intermittent application of ultrasound on the solvent extraction of gallium from a synthetic sodium hydroxide solution. The results of this test are presented in Figure 36.

For each intermittent cycle, one can identify two times, one when the ultrasound was on, and one when the ultrasound was off. When ultrasound was introduced for a particular on-time period, a large number of tiny droplets were formed, promoting very fast extraction rates. When ultrasound was discontinued in the next off-time period, the droplets tended to coalesce, and the ultrasound was introduced again. The (on + off) time cycles were continued until the extraction of gallium reached $70 \%$. It is clear that the ultrasonic energy consumption is directly proportional to both the length of the on-time period per cycle, and the total duration of the extraction.

It was found in this study that the total energy needed for the ultrasonic treatment can be greatly reduced if a higher intensity ultrasound is used. Although the higher intensity ultrasound requires more power, the time needed to produce the sonochemical effects is much shorter than 


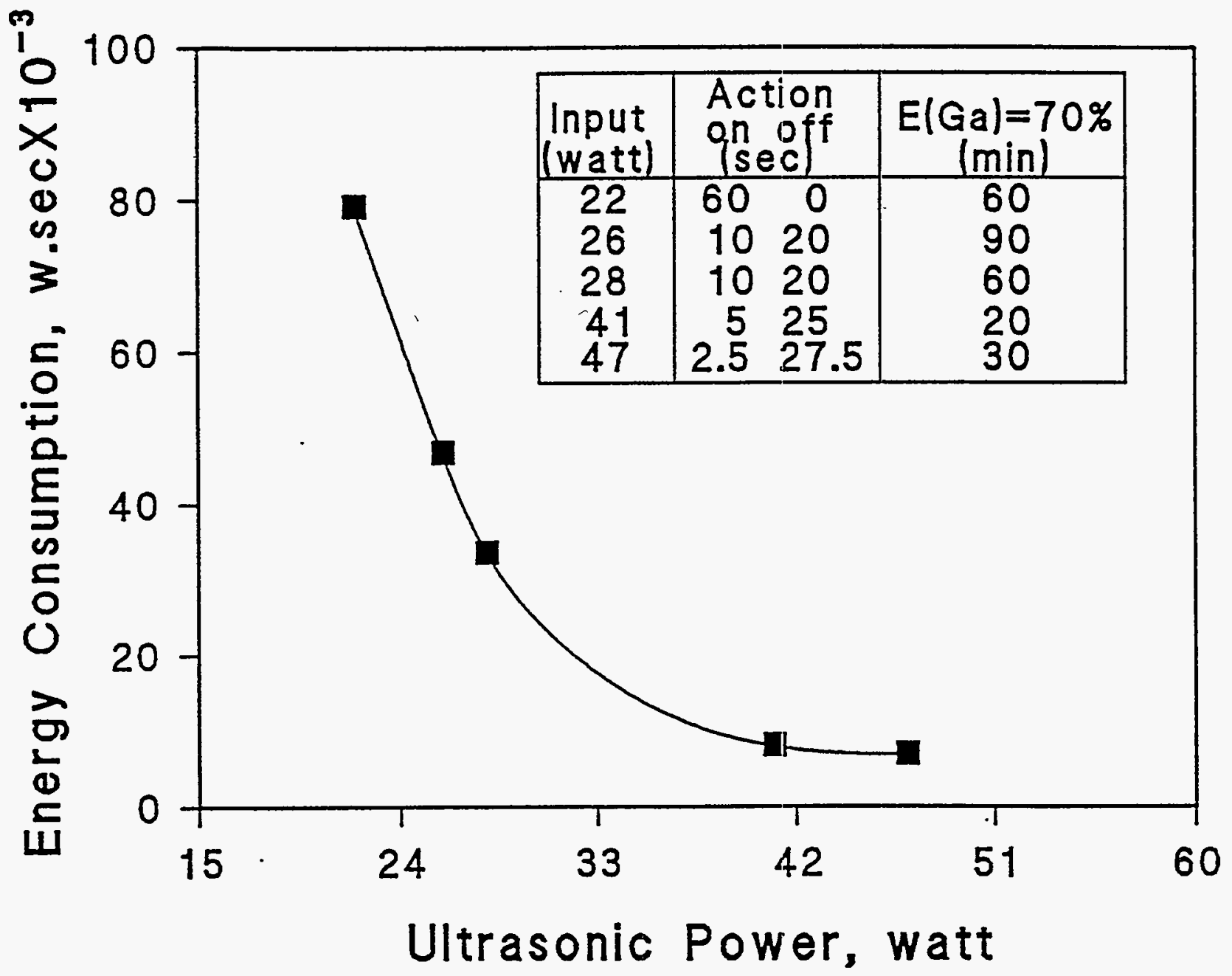

Figure 36. Energy consumption as a function of the mode of intermittent ultrasonic action for the solvent extraction of gallium from a synthetic sodium hydroxide solution.

Organic phase, (v/v): $8 \%$ Kelex 100, 10\% decanol, $82 \%$ Kermac 470B.

Aqueous phase, $(\mathrm{g} / \mathrm{L})$ : $0.15 \mathrm{Ga}, 175 \mathrm{Na}_{2} \mathrm{O}$.

System: $\mathrm{O} / \mathrm{A}=1: 1, T=25^{\circ} \mathrm{C}$.

Ultrasound: $20 \mathrm{kHz}$, indicated power levels. 
at lower intensities. In addition, for the intermittent application of the ultrasound, the use of a higher intensity allows a much shorter on-time per cycle, which also results in a lower overall energy consumption. For example, as shown in Figure 36, when the ultrasonic power input was $26 \mathrm{~W}$, and the on and off-times were 10 and $20 \mathrm{sec}$ per cycle, respectively, it took 90 minutes to extract $70 \%$ of the gallium. On the other hand, when the ultrasonic power input was $41 \mathrm{~W}$, and the on and off-times were 5 and $25 \mathrm{sec}$ per cycle, respectively, it took only $20 \mathrm{~min}$ to achieve the same level of extraction. The total energy consumption was much smaller in the second case, although a higher intensity ultrasonic treatment was used.

\section{Conclusions for Part III}

On the basis of the results described in Part III of this report, the following conclusions have been drawn:

- The use of ultrasound in the solvent extraction of gallium improves extraction rates (up to a factor of 15 ) for both synthetic and real sodium aluminate solutions.

- The solvent extraction of gallium is much faster for synthetic sodium aluminate solutions than for real solutions.

- When ultrasound is used, temperature does not have any effect on the extraction of gallium from synthetic solutions. This is contrary to the effect of temperature in the absence of ultrasound, where increased temperatures result in increased extraction rates.

- Diluents play an important role. The fastest rates of extraction are obtained with Escaid 200. The use of Aromatic 150 results in the slowest rate of extraction.

- Modifiers also have a significant effect. Ketones (2-undecanone) perform better than alcohols (decanol) and provide very rapid extraction rates even in the absence of ultrasound.

- The effects of sodium hydroxide are similar both with and without the presence of ultrasound. An increase in sodium hydroxide concentration causes an increase in extraction rates, but only up to a certain concentration limit, beyond which a further increase is detrimental. The presence of aluminum hydroxide is detrimental at all concentrations. Increasing concentrations of aluminum hydroxide results in decreasing rates of sonochemical extraction.

- Batch processes using ultrasound would be very energy intensive. The energy consumption can be significantly reduced by an intermittent application of ultrasound. 


\section{REFERENCES}

1. W. T. Richards and A. I. Loomis, "The Chemical Effects of High-Frequency Sound Waves," J. Am. Chem. Soc., 49, 1927, pp. 3086-100.

2. K. S. Suslick, "The Chemical Effects of Ultrasound," Scientific American, February 1989, pp. 80-86.

3. M. J. Ashley, "Ultrasonics in Chemical Processing," Chem. Engineer, June 1974, pp. 368-71.

4. R. M. G. Boucher, "Sonochemistry at Low and High Ultrasonic Frequencies," British Chemical Engineering, 15, 3, 1970, pp. 363-7.

5. V. A. Glembotskii and A. E. Kolchemanova, "Izd-vo Nauka," Moscow, 1967, p. 45.

6. K. S. Akopova, I. D. Dokshina, N. D. Tyutyunnik, and I. A. Yakubovich, "Changing the Flotation Properties of Placer Minerals with Ultrasound," Sb. Mosk. Inst. Stali Splavov, 60, 1970, pp. 106-18.

7. I. A Yakubovich, B. A. Agranat, O. D. Kirilov, and N. N. Khavskii, "The Applications of Ultrasonics in the Technology of Nonferrous Metals," Izvest. VUZ, Tsvet. Metall., 4, 1964, pp. 23-9.

8. R. L. Cavanagh and A. J. Last, "Potential for Sonics and Ultrasonics in Mineral Processing," Engng. Min. J., 166, May 1965, p. 98.

9. A. A. Bershitskii and B. N. Shmalei, "Question of the Kinetics of Nitric Acid Decomposition of Scheelite with Acoustical Improvement," Sb. Nauchn. Tr. - Sredneaziat. Nauchno - Issled, Proektn. Inst. Tsvetn, Metall., 11, 1975, pp. 126-131; A. A. Bershitskii, "Industrial Testing of the Use of Acoustic Vibrations in Hydrometallurgy of Tungsten," ibid., pp. 131-4.

10. M. V. Gramatikov and M. D. Ivanovskii, "Kinetics of Zinc Ferrite Dissolution in Sulfuric Acid under the Influence of Ultrasonic," J. Appl. Chem. USSR, 45, 1972, pp. 1220-23.

11. L. P. Rusikhina and L. T. Ozolin, "Effect of an Ultrasonic Field on the Leaching of Copper Ores," Mater. Vses. Konf. Fiz.-Khim. Metod. Razrab. Mestorozhd. Polez. Iskop., 1969 (published 1970), 86-108; Chem. Abs. 79:33919r.

12. B. Ya. Konyaev et al., "Izv. Vyssh. Ucheb. Zaved.," Khim. Khim. Tekhnol., 14, 6, 1971, pp. 851-4.

13. K. A Bagdasaryan, "Intensification of Gold Cyanidation Using Ultrasound," Mater. Resp. Soveshch. Neorg. Khim., 5th, 1976, p. 16; Chem. Abs. 91:110677n. 
14. V. S. Glukhov and A. V. Pronishchev, "Effect of Ultrasound on Cyaniding of GoldContaining Flotation Concentrates," Tr. Vses. Nauch. - Issled. Inst. Zolota Redk. Metal., 32, 1972, pp. 65-72; Chem. Abs. 81:40107u.

15. M. U. Kim, A. V. Umarova, and Z. R. Mansurova, "Acoustic Improvement of the Cyaniding of Gold- and Silver-Containing Ores," Tekhnol. Obogashch. Polezn. Iskopaemykh Sredn. Azii, Tashkent, 2, 1979, pp. 13-16.

16. Wayne C. Henderson and E. O. Barlow, Private Report, March 1988.

17. R. Walker, "The Role of Ultrasound in the Electrowinning and Electro-Refining of Metals," Hydrometallurgy, 4, 1979, pp. 209-215.

18. R. Walker and C. T. Walker, "New Explanation for the Hardening Effect of Ultrasound on Electrodeposits," Nature (London), 244, 1973, p. 141.

19. C. B. Kenahen and D. Schlain, Plating, 48, 1961, p. 37.

20. I. Enchev, M. Gramatikov, and N. Mikhailov, Metalurgiya (sofia), 29, 8, 1974, pp. 12-15.

21. C. Eggett, W. R. Hopkins, T. W. Garlick, and M. J. Ashley, "High Current Density Electrowinning of Copper from Ultrasonically Agitated Solutions," AIME Meeting, New York, NY, February 16-20, 1975.

22. M. V. Shostak et al., "The Effect of Ultrasound on the Ion-exchange Kinetics," Teoriya $i$ Prakt. Ionnogo Obmena. Akad: Nauk. Kaz. SSR, Tr. Resp. Soveshch., 1962, pp. 43-45; Chem. Abs. 61:12675g.

23. N. N. Khavskii et al., Collection of Reports at the All-Union Conference on Ultrasonics, 1965, pp. 187-189.

24. N. N. Khavskii, Yu. R. Smirnov, and A. A. Bershitskii, "The Uses of Ultrasonics in Nonferrous Metals Production," Tsvet. Metal., 44, 4, 1971, pp. 4-11.

25. K. L. Cheng and Z. Wang, "The Effect of Ultrasound and Mechanical Stirring on the IonExchange Kinetics," Microchimica Acta, 1982, pp. 399-406.

26. Kiyoshi Inoue, "Separation of Rare Earth Metals by Ion-exchange or Solvent Extraction," JP Pat. 86 210,132, 1986, Chem. Abs. 106:21528a.

27. Hitachi, Ltd. JP Pat. 82 119,822; 82 201,536, 1982; Toshiba Corp. JP Pat. 82 44,899, 1982.

28. The Miner's News, "Company's Tests Show Infrasonics Provide Reduced Leach Times, Other Benefits," Summer 1988, p. 6C.

29. H. A. Woodle Jr. and F. C. Vilbrandt, "Mechanism by which Ultrasonic Energy Affects Transfer Rates in Liquid-Liquid Extraction," A.I.Ch.E. Journal, 62, 1960, pp. 296-298. 
30. E. C. Chen and W. Y. Chon, "Effect of Ultrasonic Pulsation on Continuous Liquid-Liquid Extraction," CEP Symposium series 61, 77, 1967, p. 44.

31. F. Flisak and A. Perna, "The Influence of Ultrasonics on Liquid-Liquid Extraction," Ultrasonics, January 1977, pp. 27-29.

32. V. V. Vasil'ev and F. Kaveiya, "On the Possibility of Intensifying the Extraction of Chelate Compounds under the Action of Ultrasound," Vestn. Leningr. Univ., Fiz. Khim., 1, 1976, pp. 146-7; CA 84:170386g.

33. N. N. Khavskii, I. A. Yakubovich, and V. D. Tokarev, "Effect of Elastic Vibrations on Extraction Process," Sb. Mosk. Inst. Stali Splavkov, 60, 1970, pp. 161-170.

34. N. N. Khavskii et al., "Use of Ultrasonic Vibrations to Improve the Separation of Hard-ToSeparate Water-Organic Mixture in Extraction Systems with TBP," Sb. Mosk. Inst. Stali Splavov, 77, 1974, pp. 84-86; CA 82:173956n.

35. N. N. Khavskii et al., "Mechanism of the Separation of Extraction Emulsions with TBP in an Ultrasonic Field," Izv. Vyssh. Uchebn. Zaved, Tsvetn. Metall, 5, 1977, pp. 78-82; CA 88:64548j.

36. V. S. Sastri and D. J. Mackinnon, "Some Effects of Ultrasonics and their Application in Metallic Ore Processing," J. Scient. Ind. Res., 36, 1977, pp. 379-385.

37. K. S. Suslick, D. J. Casadonte, M. L. H. Green, and M. E. Thompson, "Effects of High Intensity Ultrasound on Inorganic Solids," Ultrasonics, January 25, 1987, pp. 56-59.

38. D. S. Flett and D. W. West, "Extraction of Metal Ions by Lix 63-Carboxylic Acid Mixtures," Proc. Inter. Solv. Extrac. Conf., 1, 1971, pp. 214-223.

39. D. S. Flett, M. Cox, and J. D. Heels, "Extraction of Nickel by a Hydroxyoxime/Carboxylic Acid Mixture," Proc. Inter. Solv. Extrac. Conf., SCI London, 3, 1974, pp. 2559-2575.

40. D. S. Flett, M. Cox, and J. D. Heels, "Kinetics of Nickel Extraction by Hydroxyoxime/Carboxylic Acid Mixtures," J. Inorg. Nucl. Chem., 37, 1975, pp. 2533-2537.

41. T. Tammi, "Extraction of Nickel and Cobalt with Hydroxyoxime Containing Extraction Agents," Tutkimus Tek., 4, 1976, pp. 44-45; CA 89:118498j.

42. B. G. Nyman and L. Hummelsedt, "Uses of Liquid Cation Exchange for Separation of Nickel (II) and Cobalt (II) with Simultaneous Concentration of Nickel Sulphate," Proc. Inter. Solv. Extrac. Conf., SCI London, 1, 1974, pp. 669-684.

43. L. Hummelstedt, H. E. Sund, J. Karjaluoto, L. O. Berts, and B. G. Nyman, "Use of Extractant Mixtures Containing Kelex 100 for Separation of Nickel (II) and Cobalt (II)," Proc. Inter. Solv. Extrac. Conf., Lyon, France, 1, 1974, pp. 829-848. 
44. D. S. Flett, M. Cox, and J. D. Heels, "The Extraction of Cobalt with a Proprietary Alkylated 8-Quinolinol," J. Inorg. Nucl. Chem., 37, 1975, pp. 2197-2201.

45. M. Cox and D. S. Flett, "Modern Extractants for Copper, Cobalt, and Nickel," Chem. Ind. (London), 6 1987, pp. 188-193.

46. D. S. Flett, "Solvent Extraction in Warren Spring Laboratory," Chem. Ind. (London), 6, 1977, pp. 223-4.

47. D. Bauer and J. Komornicki, "Use of Microemulsion as Organic Phase for Faster Kinetics," Proc. Inter. Solv. Extrac. Conf., 1983, pp. 315-6.

48. A. Leveque and J. Helgorsky, "The Recovery of Gallium from Bayer Process Aluminate Solution by Liquid-Liquid Extraction," Proc. Inter. Solv. Extrac. Conf., Toronto, CIM Spec., 21, 2, 1979, pp. 439-442.

49. Chen-Ming Wang, Li-Quan Zhang, J. G. Xu, J. H. Li, S. Q. Sun, and J. Z. He, "Separation of Gallium from Alkaline Aluminate Liquor Extraction from Low-Alkalinity Liquor with Kelex 100," Recent Development in Separation Science, VIII, Chapter 8, N. Li Norman and J.D. Navratil (eds.), CRC Press, 1986, pp. 251-259.

50. J. Helgorsky and A. Leveque, Braz. Patent 78 08,230, June 26, 1979.

51. D. Bauer, P. Fourre, and J. L. Sabot, (a) Eur. Patent 102,280, March 7, 1984; (b) Eur. Patent 102,882, March 14, 1984.

52. P. Fourre and D. Bauer, "Enhancement of the Extraction Rate of Gallium by the 7-(1-vinyl 3,3,5,5 tetramethylhexyl)-8-quinolinol Using a Microemulsion as Organic Phase," Solv. Extrac. Ion Exch., 1, 1983, pp. 465-483.

53. D. Bauer and Y. Pescher-Cluzeau, "Liquid-Liquid Extraction of Aluminum and Gallium with 5-Substituted 8-Hydroxyquinolines," Hydrometallurgy, 18, 1987, pp. 243-253.

54. T. Sato and H. Oishi, "Solvent Extraction of Gallium (III) from Sodium Hydroxide Solution by Alkylated Hydroxyquinoline," Hydrometallurgy, 1986, pp. 315-24.

55. T. Sato, T. Yoshino, T. Nakamura, and T. Kudo, "The Kinetics of Aluminum (III) Extraction from Sulphuric Acid Solutions by di-(2-ethyl-hexyl)-Phosphoric Acid," J. Inorg. Nucl. Chem., 40, 1978, pp. 1571-4; 41, 1979, pp. 731-4.

56. P. Gareil, S. De Beler, and D. Bauer, "Composition Analysis of Kelex 100, an Industrial Chelating Extractant, by Liquid Chromatography and Mass Spectrometry," Hydrometallurgy, 22, 1989, pp. 239-248.

57. B. Pesic and T. Zhou, "Recovering Gallium with Kelex 100," J. Metals, July 1988, pp. 24-26. 
58. R. J. Moolenaar, J. C. Evans, and L. D. McKeever, "The Structure of the Aluminate Ion in Solutions at High pH," J. Phys. Chem., 74, 1970, pp. 3629-36.

59. T. Sato, "Liquid-Liquid Extraction of Gallium (III) and Aluminum (III) from Sodium Hydroxide Solutions by 7-Alkylated 8-Hydroxyquinoline," Chemical Separations, Vol. 1: Principles, 1st International Conference on Separation Science and Technology, New York, NY, April 1986, C. J. King and J. D. Navratil (eds.), Litarvan Literature, Denver, CO, 1986, pp. 285-94.

60. N. G. Dovbysh, Yu. A. Volokhov, N. I. Eremin, and V. E. Mironov, "An Ultrasonic Study of Aluminate Solutions," Russian J. Phys. Chem., 50, 1976, pp. 1461-4.

61. M. Eigen, "Fast Elementary Steps in Chemical Reaction Mechanisms," Pure Appl. Chem., June 1963, pp. 97-115.

62. J. C. Hindman and A. Svirmickas, J. Phys. Chem., 77, 1973, p. 2847.

63. N. G. Dovbysh, A. M. Sazonov, Yu. A. Volokhov, and V. E. Mironov, "Hydration of Hydroxo Complexes of Aluminum (III) and Gallium (III)," Russian J. Phys. Chem., 52, 1978, pp. $1578-80$.

64. P. Fourre and D. Bauer, "Microemulsions in the Extraction of Gallium with 7-(1-Ethenyl3,3,5,5-tetramethylhexyl)-8-quinolinol from Aluminate Solutions," Anal. Chem., 55, 1983, pp. 662-7.

65. T. Sato and H. Oishi, "Solvent Extraction of Gallium (III) from Sodium Hydroxide Solution by Alkylated Hydroxyquinoline," Hydrometallurgy, 16, 1986, pp. 315-24. 